\title{
ASYMPTOTIC ANALYSIS OF TRANSPORT EQUATION IN ANNULUS
}

\author{
LEI WU, XIONGFENG YANG, AND YAN GUO
}

\begin{abstract}
We consider the diffusive limit of a steady neutron transport equation with one-speed velocity in a two-dimensional annulus. A classical theorem in 1 states that the solution can be approximated in $L^{\infty}$ by the leading order interior solution plus the corresponding Knudsen layers in the diffusive limit. In this paper, we construct a counterexample of this result via a different boundary layer expansion with geometric correction.
\end{abstract}

Keywords: $\epsilon$-Milne problem, Knudsen layer, Geometric correction.

\section{CONTENTS}

List of Figures

1. Introduction and Notation

1.1. Problem Formulation

1.2. Background and Idea

1.3. Main Results

1.4. Notation and Structure

2. Asymptotic Analysis

2.1. Interior Expansion

2.2. Milne Expansion

2.3. $\epsilon$-Milne Expansion with Geometric Correction

3. $\epsilon$-Milne Problem with Geometric Correction

3.1. $L^{2}$ Estimates

3.2. $L^{\infty}$ Estimates

3.3. Exponential Decay

3.4. Maximum Principle

4. Remainder Estimate

4.1. Preliminaries

4.2. Uniform $L^{2}$ Estimate

4.3. $L^{\infty}$ Estimate

5. Diffusive Limit

References

\section{List OF Figures}

1 Characteristics of Flat Milne Problem for $f_{0}$

2 Characteristics of $\epsilon$-Milne Problem in Convex Domain for $f_{+}$

3 Characteristics of $\epsilon$-Milne Problem in Non-Convex Domain for $f_{-}$

L. Wu is supported by NSF grant 0967140. X.F. Yang is supported by NNSF of China under the Grant 11171212 and the SJTU's SMC Projection A. Y. Guo is supported in part by NSFC grant 10828103, NSF grant 1209437, Simon Research Fellowship and BICMR.. 


\section{INTRODUCTION AND Notation}

1.1. Problem Formulation. We consider a homogeneous isotropic steady neutron transport equation with one-speed velocity $\Sigma=\left\{\vec{w}=\left(w_{1}, w_{2}\right): \vec{w} \in \mathcal{S}^{1}\right\}$ in a two-dimensional annulus $\Omega=\left\{\vec{x}=\left(x_{1}, x_{2}\right): 0<\right.$ $\left.R_{-}<|\vec{x}|<R_{+}<\infty\right\}$ as

$$
\left\{\begin{aligned}
\epsilon \vec{w} \cdot \nabla_{x} u^{\epsilon}+u^{\epsilon}-\bar{u}^{\epsilon} & =0 \text { in } \Omega, \\
u^{\epsilon}\left(\vec{x}_{0}, \vec{w}\right) & =g_{ \pm}\left(\vec{x}_{0}, \vec{w}\right) \text { for } \vec{w} \cdot \vec{n}<0 \text { and }\left|\vec{x}_{0}\right|=R_{ \pm},
\end{aligned}\right.
$$

where

$$
\bar{u}^{\epsilon}(\vec{x})=\frac{1}{2 \pi} \int_{\mathcal{S}^{1}} u^{\epsilon}(\vec{x}, \vec{w}) \mathrm{d} \vec{w} .
$$

and $\vec{n}$ is the outward normal vector on $\partial \Omega, 0<\epsilon<<1$ is the Knudsen number. The in-flow boundary condition is given on the two circles $R_{ \pm}$. In this paper, we will study the diffusive limit of the solution $u^{\epsilon}$ to (1.1) as $\epsilon \rightarrow 0$.

In the physical space, we consider the two-dimensional annulus $\Omega=\left\{\vec{x}=\left(x_{1}, x_{2}\right): 0<R_{-}<|\vec{x}|<\right.$ $\left.R_{+}<\infty\right\}$. Its boundary $\partial \Omega$ includes the inner boundary and outer boundary, that is, $\partial \Omega=\partial \Omega_{-} \cup \partial \Omega_{+}$ where

$$
\begin{aligned}
& \partial \Omega_{-}=\left\{\vec{x}:|\vec{x}|=R_{-}\right\}, \\
& \partial \Omega_{+}=\left\{\vec{x}:|\vec{x}|=R_{+}\right\} .
\end{aligned}
$$

Based on the flow direction, we can divide the boundary in phase space $\Gamma=\{(\vec{x}, \vec{w}): \vec{x} \in \partial \Omega\}$ into the in-flow boundary $\Gamma^{-}$, the out-flow boundary $\Gamma^{+}$, and the grazing set $\Gamma^{0}$ as

$$
\begin{aligned}
\Gamma^{-} & =\{(\vec{x}, \vec{w}): \vec{x} \in \partial \Omega, \vec{w} \cdot \vec{n}<0\}, \\
\Gamma^{+} & =\{(\vec{x}, \vec{w}): \vec{x} \in \partial \Omega, \vec{w} \cdot \vec{n}>0\}, \\
\Gamma^{0} & =\{(\vec{x}, \vec{w}): \vec{x} \in \partial \Omega, \vec{w} \cdot \vec{n}=0\} .
\end{aligned}
$$

So $\Gamma=\Gamma^{+} \cup \Gamma^{-} \cup \Gamma^{0}$. For the in-flow boundary condition, the boundary value is only given on $\Gamma^{-}$.

A classical result in [1] states that the solution $u^{\epsilon}$ of (1.1) satisfies

$$
\left\|u^{\epsilon}-U_{0}-\mathscr{U}_{+, 0}-\mathscr{U}_{-, 0}\right\|_{L^{\infty}}=O(\epsilon)
$$

where $\mathscr{U}_{ \pm, 0}$ is the Knudsen layer to the Milne problem (2.28) while $U_{0}$ is the corresponding interior solution to the Laplace equation (2.29). The goal of this paper is to construct a counterexample to such a result in an annulus.

1.2. Background and Idea. The study of neutron transport equation can date back to 1960s. Since then, this type of problems have been extensively studied in many different settings: steady or unsteady, linear or nonlinear, strong solution or weak solution, etc, (see [5], [4, [6, 7], 8], 9], [10, [11, 12]). Among all these variations, one of the simplest but most important models - steady neutron transport equation with one-speed velocity in bounded domains, where the boundary layer effect shows up, has long been believed to be satisfactorily solved since Bensoussan, Lions and Papanicolaou published their remarkable paper [1] in 1979.

The basic idea in [1] is to consider the boundary layer $f_{0}(\eta, \phi)$ satisfies that in the domain $(\eta, \phi) \in$ $[0, \infty) \times[-\pi, \pi)$,

$$
\left\{\begin{aligned}
\sin \phi \frac{\partial f_{0}}{\partial \eta}+f_{0}-\bar{f}_{0} & =S_{0}(\eta, \phi), \\
f_{0}(0, \phi) & =h_{0}(\phi) \text { for } \sin \phi>0, \\
\lim _{\eta \rightarrow \infty} f_{0}(\eta, \phi) & =f_{0, \infty},
\end{aligned}\right.
$$

where $\eta$ denotes the normal variable and $\phi$ the velocity variable. This is the well-known Milne problem and $f_{0}$ can be shown to be well-posedness and decays exponentially fast to $f_{0, \infty}$ in $L^{\infty}$.

However, in [13] the authors pointed out that the construction of boundary layer in [1] based on Milne problem will break down due to singularity near the grazing set. This brings our attention back to the starting point and we have to reexamine all of the related results. Also, in [13, a new approach was introduced and shown to be effective when the domain is a two-dimensional plate. 
The central idea of constructing boundary layer is to consider so-called $\epsilon$-Milne problem with geometric correction. For annulus, the boundary layer $f_{+}(\eta, \phi)$ near outer circle satisfies

$$
\left\{\begin{aligned}
\sin \phi \frac{\partial f_{+}}{\partial \eta}-\frac{\epsilon}{R_{+}-\epsilon \eta} \cos \phi \frac{\partial f_{+}}{\partial \phi}+f_{+}-\bar{f}_{+} & =S_{+}(\eta, \phi), \\
f_{+}(0, \phi) & =h_{+}(\phi) \text { for } \sin \phi>0, \\
\lim _{\eta \rightarrow \infty} f_{+}(\eta, \phi) & =f_{+, \infty},
\end{aligned}\right.
$$

Simply speaking, the boundary layer of outer circle is similar to the boundary in a plate. This problem has been extensively studied in [13, Section 4] and we know the solution $f_{+}$is well-posedness and decays exponentially fast to $f_{+, \infty}$.

However, for inner circle, we must consider the boundary $f_{-}(\eta, \phi)$ satisfying

$$
\left\{\begin{aligned}
\sin \phi \frac{\partial f_{-}}{\partial \eta}+\frac{\epsilon}{R_{-}+\epsilon \eta} \cos \phi \frac{\partial f_{-}}{\partial \phi}+f_{-}-\bar{f}_{-} & =S_{-}(\eta, \phi), \\
f_{-}(0, \phi) & =h_{-}(\phi) \text { for } \sin \phi>0, \\
\lim _{\eta \rightarrow \infty} f_{-}(\eta, \phi) & =f_{-, \infty},
\end{aligned}\right.
$$

The proof in 13 relies on the analysis along the characteristics. However, this changed sign of the second term in (1.11) will greatly affect the shape of characteristics, which is shown by Figures 1, 2 and 3 ,

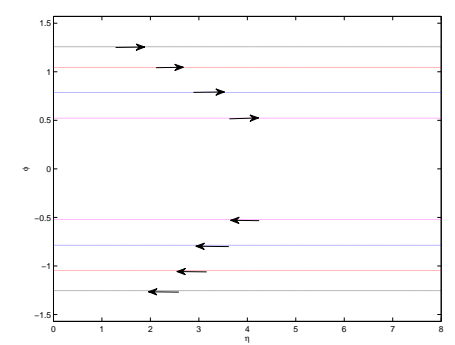

FiguRE 1. Characteristics of Flat Milne Problem for $f_{0}$

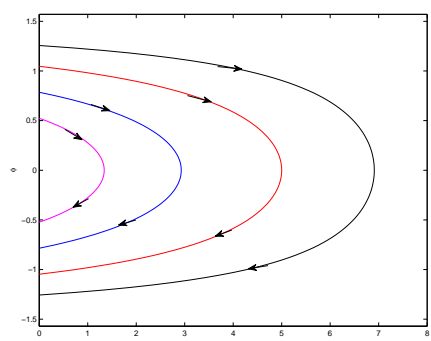

Figure 2. Characteristics of $\epsilon$-Milne Problem in Convex Domain for $f_{+}$

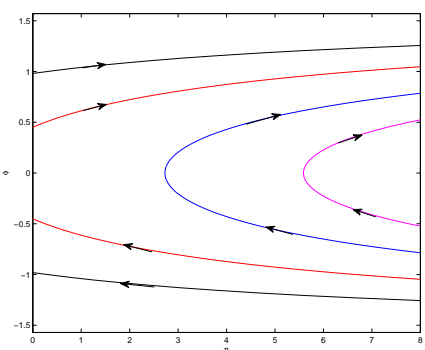

Figure 3. Characteristics of $\epsilon$-Milne Problem in Non-Convex Domain for $f_{-}$

This adds new difficulties to our estimate and we have to resort to different formulation to bound $f_{-}$.

1.3. Main Results. We will use the transformation in (2.13) and (2.41). That is, we define $\vec{x}=(r \cos \theta, r \sin \theta)$ with $R_{-} \leq r \leq R_{+}$and $-\pi \leq \theta \leq \pi$ while $\vec{w}=(-\sin \xi, \cos \xi)$ with $-\pi \leq \theta \leq \pi$. We also define the $\phi=\theta+\xi$. The boundary value is given by $\tilde{g}_{ \pm}(\theta, \phi)=g_{ \pm}\left(\vec{x}_{0}, \vec{w}\right)$. For convenience, we denote the boundary condition as $g_{ \pm}(\theta, \phi)$. Then, the diffusive limit is stated as follows:

Theorem 1.1. Assume $g_{ \pm}\left(\vec{x}_{0}, \vec{w}\right) \in C^{3}\left(\Gamma^{-}\right)$. Then there exists a unique solution $u^{\epsilon}(\vec{x}, \vec{w}) \in L^{\infty}\left(\Omega \times \mathcal{S}^{1}\right)$ for the steady neutron transport equation (1.1). Furthermore, it satisfies

$$
\left\|u^{\epsilon}-U_{0}^{\epsilon}-\mathscr{U}_{+, 0}^{\epsilon}-\mathscr{U}_{-, 0}^{\epsilon}\right\|_{L^{\infty}}=O(\epsilon)
$$


where the interior solution $U_{0}^{\epsilon}$ and boundary layer $\mathscr{U}_{ \pm, 0}^{\epsilon}$ are defined in 2.54) and (2.53). In particular, if $\left.g_{+}(\phi, \theta)\right)=0$ and $g_{-}(\theta, \phi)=\cos \phi$, then there exists a positive constant $C>0$ such that

$$
\left\|u^{\epsilon}-U_{0}-\mathscr{U}_{+, 0}-\mathscr{U}_{-, 0}\right\|_{L^{\infty}} \geq C>0
$$

when $\epsilon$ is sufficiently small, where $U_{0}$ and $\mathscr{U}_{ \pm, 0}$ are defined in (2.29) and (2.28).

1.4. Notation and Structure. Throughout this paper, $C>0$ denotes a constant only depends on the parameter $\Omega$, but does not depend on the given data. It is referred as universal and can is different from one line to another. When we write $C(z)$, it means a certain positive constant depending on the quantity $z$.

Our paper is organized as follows: In Section 2, we first present the asymptotic analysis of both the interior solution and boundary layer; In Section 3, we give a complete analysis of the $\epsilon$-Milne problem with geometric correction; In Section 4, we establish the $L^{\infty}$ remainder estimate; finally, in Section 5, we prove the diffusive limit of the solution, i.e. Theorem 1.1.

\section{Asymptotic Analysis}

2.1. Interior Expansion. We define the interior expansion as follows:

$$
U(\vec{x}, \vec{w}) \sim \sum_{k=0}^{\infty} \epsilon^{k} U_{k}(\vec{x}, \vec{w}) .
$$

Plugging (2.1) into the equation (1.1) and comparing the order of $\epsilon$, the functions $U_{k}(k=0,1,2 \cdots$,$) should$ satisfy

$$
\begin{aligned}
U_{0}-\bar{U}_{0} & =0, \\
U_{1}-\bar{U}_{1} & =-\vec{w} \cdot \nabla_{x} U_{0}, \\
U_{2}-\bar{U}_{2} & =-\vec{w} \cdot \nabla_{x} U_{1}, \\
\cdots & \\
U_{k}-\bar{U}_{k} & =-\vec{w} \cdot \nabla_{x} U_{k-1} .
\end{aligned}
$$

The following analysis reveals the equation satisfied by $U_{k}$ :

The equalities (2.2) and (2.3) could be rewritten as

$$
U_{1}=\bar{U}_{1}-\vec{w} \cdot \nabla_{x} \bar{U}_{0} .
$$

Thus, from (2.6) into (2.4), we get

$$
U_{2}-\bar{U}_{2}=-\vec{w} \cdot \nabla_{x}\left(\bar{U}_{1}-\vec{w} \cdot \nabla_{x} \bar{U}_{0}\right)=-\vec{w} \cdot \nabla_{x} \bar{U}_{1}+|\vec{w}|^{2} \Delta_{x} \bar{U}_{0}+2 w_{1} w_{2} \partial_{x_{1} x_{2}} \bar{U}_{0} .
$$

Integrating (2.7) over $\vec{w} \in \mathcal{S}^{1}$, we achieve the final form

$$
\Delta_{x} \bar{U}_{0}=0
$$

which further implies $U_{0}(\vec{x}, \vec{w})$ satisfies the equation

$$
\left\{\begin{aligned}
U_{0} & =\bar{U}_{0} \\
\Delta_{x} U_{0} & =0
\end{aligned}\right.
$$

Similarly, we can derive $U_{k}(\vec{x}, \vec{w})$ for $k \geq 1$ satisfies

$$
\left\{\begin{aligned}
U_{k} & =\bar{U}_{k}-\vec{w} \cdot \nabla_{x} U_{k-1} \\
\Delta_{x} \bar{U}_{k} & =0
\end{aligned}\right.
$$


2.2. Milne Expansion. In general, the value of (2.1) on the boundary is different from the in-flow boundary condition in (1.1). In order to match the boundary condition, we need to give the boundary layer expansion. Here we firstly recall the idea of this expansion in [1, pp.136] in the following several substitutions:

Substitution 1: We consider the substitution into quasi-polar coordinates $\left(x_{1}, x_{2}, \vec{w}\right) \rightarrow\left(\mu_{ \pm}, \theta, \vec{w}\right)$ with $\left(\mu_{ \pm}, \theta, \vec{w}\right) \in\left[0, R_{+}-R_{-}\right] \times[-\pi, \pi) \times \mathcal{S}^{1}$ defined as

$$
\left\{\begin{aligned}
x_{1} & =\left(R_{ \pm} \mp \mu_{ \pm}\right) \cos \theta \\
x_{2} & =\left(R_{ \pm} \mp \mu_{ \pm}\right) \sin \theta \\
w_{1} & =w_{1} \\
w_{2} & =w_{2}
\end{aligned}\right.
$$

Here $\mu_{ \pm}$denotes the distance to the boundary $\partial \Omega_{ \pm}$and $\theta$ is the space angular variable. In these new variables, we also denote the solution as $u^{e}\left(\mu_{ \pm}, \theta, \vec{w}\right)$. Then, the equation (1.1) can be rewritten as

$$
\left\{\begin{array}{l}
\mp \epsilon\left(w_{1} \cos \theta+w_{2} \sin \theta\right) \frac{\partial u^{\epsilon}}{\partial \mu_{ \pm}}-\frac{\epsilon\left(w_{1} \sin \theta-w_{2} \cos \theta\right)}{R_{ \pm} \mp \mu_{ \pm}} \frac{\partial u^{\epsilon}}{\partial \theta}+u^{\epsilon}-\frac{1}{2 \pi} \int_{\mathcal{S}^{1}} u^{\epsilon} \mathrm{d} \vec{w}=0, \\
u^{\epsilon}\left(0, \theta, w_{1}, w_{2}\right)=g_{ \pm}\left(\theta, w_{1}, w_{2}\right) \text { for } \quad \pm\left(w_{1} \cos \theta+w_{2} \sin \theta\right)<0 .
\end{array}\right.
$$

Substitution 2: We further define the stretched variable $\eta_{ \pm}$by making the scaling transform for $u^{\epsilon}\left(\mu_{ \pm}, \theta, w_{1}, w_{2}\right) \rightarrow$ $u^{\epsilon}\left(\eta_{ \pm}, \theta, w_{1}, w_{2}\right)$ with $\left(\eta_{ \pm}, \theta, w_{1}, w_{2}\right) \in\left[0,\left(R_{+}-R_{-}\right) / \epsilon\right] \times[-\pi, \pi) \times \mathcal{S}^{1}$ as

$$
\left\{\begin{aligned}
\eta_{ \pm} & =\mu_{ \pm} / \epsilon \\
\theta & =\theta \\
w_{1} & =w_{1} \\
w_{2} & =w_{2},
\end{aligned}\right.
$$

which implies

$$
\frac{\partial u^{\epsilon}}{\partial \mu_{ \pm}}=\frac{1}{\epsilon} \frac{\partial u^{\epsilon}}{\partial \eta_{ \pm}}
$$

Then equation (1.1) is transformed into

$$
\left\{\begin{array}{l}
\mp\left(w_{1} \cos \theta+w_{2} \sin \theta\right) \frac{\partial u^{\epsilon}}{\partial \eta_{ \pm}}-\frac{\epsilon\left(w_{1} \sin \theta-w_{2} \cos \theta\right)}{R_{ \pm} \mp \epsilon \eta_{ \pm}} \frac{\partial u^{\epsilon}}{\partial \theta}+u^{\epsilon}-\frac{1}{2 \pi} \int_{\mathcal{S}^{1}} u^{\epsilon} \mathrm{d} \vec{w}=0 \\
u^{\epsilon}\left(0, \theta, w_{1}, w_{2}\right)=g_{ \pm}\left(\theta, w_{1}, w_{2}\right) \text { for } \quad \pm\left(w_{1} \cos \theta+w_{2} \sin \theta\right)<0
\end{array}\right.
$$

Substitution 3: Define the velocity substitution for $u^{\epsilon}\left(\eta_{ \pm}, \theta, w_{1}, w_{2}\right) \rightarrow u^{\epsilon}\left(\eta_{ \pm}, \theta, \xi\right)$ with $\left(\eta_{ \pm}, \theta, \xi\right) \in\left[0,\left(R_{+}-\right.\right.$ $\left.\left.R_{-}\right) / \epsilon\right] \times[-\pi, \pi) \times[-\pi, \pi)$ as

$$
\left\{\begin{aligned}
\eta_{ \pm} & =\eta_{ \pm}, \\
\theta & =\theta, \\
w_{1} & =-\sin \xi \\
w_{2} & =-\cos \xi .
\end{aligned}\right.
$$

Here $\xi$ denotes the velocity angular variable. We have the succinct form for (1.1) as

$$
\left\{\begin{array}{l} 
\pm \sin (\theta+\xi) \frac{\partial u^{\epsilon}}{\partial \eta_{ \pm}}-\frac{\epsilon \cos (\theta+\xi)}{R_{ \pm} \mp \epsilon \eta_{ \pm}} \frac{\partial u^{\epsilon}}{\partial \theta}+u^{\epsilon}-\frac{1}{2 \pi} \int_{-\pi}^{\pi} u^{\epsilon} \mathrm{d} \xi=0 \\
u^{\epsilon}(0, \theta, \xi)=g_{ \pm}(\theta, \xi) \text { for } \pm \sin (\theta+\xi)>0
\end{array}\right.
$$

We now define the Milne expansion of boundary layer as follows:

$$
\mathscr{U}_{ \pm}\left(\eta_{ \pm}, \theta, \phi\right) \sim \sum_{k=0}^{\infty} \epsilon^{k} \mathscr{U}_{ \pm, k}\left(\eta_{ \pm}, \theta, \phi\right),
$$


where $\mathscr{U}_{ \pm, k}$ can be determined by comparing the order of $\epsilon$ via plugging (2.18) into the equation (2.17). Thus, in a neighborhood of the boundary, we have

$$
\begin{aligned}
& \pm \sin (\theta+\xi) \frac{\partial \mathscr{U}_{ \pm, 0}}{\partial \eta_{ \pm}}+\mathscr{U}_{ \pm, 0}-\overline{\mathscr{U}}_{ \pm, 0}=0 \\
& \pm \sin (\theta+\xi) \frac{\partial \mathscr{U}_{ \pm, 1}}{\partial \eta_{ \pm}}+\mathscr{U}_{ \pm, 1}-\overline{\mathscr{U}}_{ \pm, 1}=\frac{\cos (\theta+\xi)}{R_{ \pm} \mp \epsilon \eta_{ \pm}} \frac{\partial \mathscr{U}_{ \pm, 0}}{\partial \theta}, \\
& \ldots \sin (\theta+\xi) \frac{\partial \mathscr{U}_{ \pm, k}}{\partial \eta_{ \pm}}+\mathscr{U}_{ \pm, k}-\overline{\mathscr{U}}_{ \pm, k}=\frac{\cos (\theta+\xi)}{R_{ \pm} \mp \epsilon \eta_{ \pm}} \frac{\partial \mathscr{U}_{ \pm, k-1}}{\partial \theta},
\end{aligned}
$$

where

$$
\overline{\mathscr{U}}_{ \pm, k}\left(\eta_{ \pm}, \theta\right)=\frac{1}{2 \pi} \int_{-\pi}^{\pi} \mathscr{U}_{ \pm, k}\left(\eta_{ \pm}, \theta, \xi\right) \mathrm{d} \xi
$$

We hope that the solution is formulated from the interior solution and the boundary layer solution. So it should satisfy the boundary condition of (1.1). The boundary condition expansion derives to

$$
\begin{aligned}
U_{0}+\mathscr{U}_{ \pm, 0} & =g_{ \pm}, \\
U_{1}+\mathscr{U}_{ \pm, 1} & =0, \\
\ldots & \\
U_{k}+\mathscr{U}_{ \pm, k} & =0 .
\end{aligned}
$$

The construction of $U_{k}$ and $\mathscr{U}_{ \pm, k}$ in [1] can be summarized as follows:

Step 1: Construction of $\mathscr{U}_{ \pm, 0}$ and $U_{0}$.

To deal with the sigularity according to $r$, we define the cut-off function $\psi$ and $\psi_{0}$ as

$$
\begin{gathered}
\psi(\mu)= \begin{cases}1 & 0 \leq \mu \leq 1 / 2\left(R_{+}-R_{-}\right), \\
0 & 3 / 4\left(R_{+}-R_{-}\right) \leq \mu \leq \infty .\end{cases} \\
\psi_{0}(\mu)= \begin{cases}1 & 0 \leq \mu \leq 1 / 4\left(R_{+}-R_{-}\right), \\
0 & 3 / 8\left(R_{+}-R_{-}\right) \leq \mu \leq \infty .\end{cases}
\end{gathered}
$$

Then the zeroth order boundary layer solution is defined as

$$
\left\{\begin{aligned}
\mathscr{U}_{ \pm, 0}\left(\eta_{ \pm}, \theta, \xi\right) & =\psi_{0}\left(\epsilon \eta_{ \pm}\right)\left(f_{ \pm, 0}\left(\eta_{ \pm}, \theta, \xi\right)-f_{ \pm, 0}(\infty, \theta)\right) \\
\pm \sin (\theta+\xi) \frac{\partial f_{ \pm, 0}}{\partial \eta_{ \pm}}+f_{ \pm, 0}-\bar{f}_{ \pm, 0} & =0,0<\eta_{ \pm}<\infty \\
f_{ \pm, 0}(0, \theta, \xi) & =g_{ \pm}(\theta, \xi) \text { for } \pm \sin (\theta+\xi)>0 \\
\lim _{\eta_{ \pm} \rightarrow \infty} f_{ \pm, 0}\left(\eta_{ \pm}, \theta, \xi\right) & =f_{ \pm, 0}(\infty, \theta)
\end{aligned}\right.
$$

Assuming $g_{ \pm} \in L^{\infty}\left(\Gamma^{-}\right)$, by Theorem 3.12, we can show there exist unique solutions $f_{ \pm, 0}\left(\eta_{ \pm}, \theta, \xi\right) \in$ $L^{\infty}([0, \infty) \times[-\pi, \pi) \times[-\pi, \pi))$. Hence, $\mathscr{U}_{ \pm, 0}$ is well-defined. Then we define the zeroth order interior solution as

$$
\left\{\begin{aligned}
U_{0} & =\bar{U}_{0}, \\
\Delta_{x} \bar{U}_{0} & =0 \text { in } \Omega, \\
\bar{U}_{0} & =f_{ \pm, 0}(\infty, \theta) \text { on } \partial \Omega_{ \pm} .
\end{aligned}\right.
$$

Step 2: Construction of $\mathscr{U}_{ \pm, 1}$ and $U_{1}$. 
Let $\vec{x}_{ \pm}=R_{ \pm}(\cos \theta, \sin \theta)$ and $\vec{w}=(-\sin \xi, \cos \xi)$. Then, we define the first order boundary layer solution as

$$
\left\{\begin{aligned}
\mathscr{U}_{ \pm, 1}\left(\eta_{ \pm}, \theta, \xi\right) & =\psi_{0}\left(\epsilon \eta_{ \pm}\right)\left(f_{ \pm, 1}\left(\eta_{ \pm}, \theta, \xi\right)-f_{ \pm, 1}(\infty, \theta)\right) \\
\pm \sin (\theta+\xi) \frac{\partial f_{ \pm, 1}}{\partial \eta_{ \pm}}+f_{ \pm, 1}-\bar{f}_{ \pm, 1} & =\cos (\theta+\xi) \frac{\psi\left(\epsilon \eta_{ \pm}\right)}{R_{ \pm} \mp \epsilon \eta_{ \pm}} \frac{\partial \mathscr{U}_{ \pm, 0}}{\partial \theta} \\
f_{ \pm, 1}(0, \theta, \xi) & =\vec{w} \cdot \nabla_{x} U_{0}\left(\vec{x}_{ \pm}, \vec{w}\right) \text { for } \pm \sin (\theta+\xi)>0 \\
\lim _{\eta_{ \pm} \rightarrow \infty} f_{ \pm, 1}\left(\eta_{ \pm}, \theta, \xi\right) & =f_{ \pm, 1}(\infty, \theta) .
\end{aligned}\right.
$$

At the same time, we define the first order interior solution as

$$
\left\{\begin{aligned}
U_{1} & =\bar{U}_{1}-\vec{w} \cdot \nabla_{x} U_{0}, \\
\Delta_{x} \bar{U}_{1} & =0 \text { in } \Omega, \\
\bar{U}_{1} & =f_{ \pm, 1}(\infty, \theta) \text { on } \partial \Omega_{ \pm} .
\end{aligned}\right.
$$

Step 3: Generalization to arbitrary $k$.

Similar to above procedure, we can define the $k^{t h}$ order boundary layer solution as

$$
\left\{\begin{aligned}
\mathscr{U}_{ \pm, k}\left(\eta_{ \pm}, \theta, \xi\right) & =\psi_{0}\left(\epsilon \eta_{ \pm}\right)\left(f_{ \pm, k}\left(\eta_{ \pm}, \theta, \xi\right)-f_{ \pm, k}(\infty, \theta)\right) \\
\pm \sin (\theta+\xi) \frac{\partial f_{ \pm, k}}{\partial \eta_{ \pm}}+f_{ \pm, k}-\bar{f}_{ \pm, k} & =\cos (\theta+\xi) \frac{\psi\left(\epsilon \eta_{ \pm}\right)}{R_{ \pm} \mp \epsilon \eta_{ \pm}} \frac{\partial \mathscr{U}_{ \pm, k-1}}{\partial \theta} \\
f_{ \pm, k}(0, \theta, \xi) & =\vec{w} \cdot \nabla_{x} U_{k-1}\left(\vec{x}_{ \pm}, \vec{w}\right) \text { for } \pm \sin (\theta+\xi)>0 \\
\lim _{\eta_{ \pm} \rightarrow \infty} f_{ \pm, k}\left(\eta_{ \pm}, \theta, \xi\right) & =f_{ \pm, k}(\infty, \theta) .
\end{aligned}\right.
$$

Define the $k^{t h}$ order interior solution as

$$
\left\{\begin{aligned}
U_{k} & =\bar{U}_{k}-\vec{w} \cdot \nabla_{x} U_{k-1}, \\
\Delta_{x} \bar{U}_{k} & =0 \text { in } \Omega, \\
\bar{U}_{k} & =f_{ \pm, k}(\infty, \theta) \text { on } \partial \Omega_{ \pm} .
\end{aligned}\right.
$$

Combing the above discussion, we are able to prove the following result:

Theorem 2.1. Assume $g_{ \pm} \in L^{\infty}\left(\Gamma^{-}\right)$are sufficiently smooth. Then there exits a unique solution $u^{\epsilon}(\vec{x}, \vec{w}) \in$ $L^{\infty}\left(\Omega \times \mathcal{S}^{1}\right)$ for the steady neutron transport equation (1.1), which satisfies

$$
\left\|u^{\epsilon}-U_{0}-\mathscr{U}_{+, 0}-\mathscr{U}_{-, 0}\right\|_{L^{\infty}}=O(\epsilon) .
$$

Our work begins with a crucial observation that based on Remark 3.15, the existence of solution $f_{ \pm, 1}$ requires the source term

$$
\cos (\theta+\xi) \frac{\psi\left(\epsilon \eta_{ \pm}\right)}{R_{ \pm} \mp \epsilon \eta_{ \pm}} \frac{\partial \mathscr{U}_{ \pm, 0}}{\partial \theta} \in L^{\infty}([0, \infty) \times[-\pi, \pi) \times[-\pi, \pi)) .
$$

Since the support of $\psi\left(\epsilon \eta_{ \pm}\right)$depends on $\epsilon$, by (2.28), this in turn requires

$$
\frac{\partial}{\partial \theta}\left(f_{ \pm, 0}\left(\eta_{ \pm}, \theta, \xi\right)-f_{ \pm, 0}(\infty, \theta)\right) \in L^{\infty}([0, \infty) \times[-\pi, \pi) \times[-\pi, \pi))
$$

We can check that $Z_{ \pm}=\partial_{\theta}\left(f_{ \pm, 0}-f_{ \pm, 0}(\infty, \theta)\right)$ satisfy

$$
\left\{\begin{aligned}
\pm \sin (\theta+\xi) \frac{\partial Z_{ \pm}}{\partial \eta}+Z_{ \pm}-\bar{Z}_{ \pm} & =-\cos (\theta+\xi) \frac{\partial f_{ \pm, 0}}{\partial \eta_{ \pm}} \\
Z_{ \pm}(0, \theta, \xi) & =\frac{\partial g_{ \pm}(\theta, \xi)}{\partial \theta}-\frac{\partial f_{ \pm, 0}(\infty, \theta)}{\partial \theta} \text { for } \pm \sin (\theta+\xi)>0 \\
\lim _{\eta_{ \pm} \rightarrow \infty} Z_{ \pm}\left(\eta_{ \pm}, \theta, \xi\right) & =Z_{ \pm}(\infty, \theta) .
\end{aligned}\right.
$$

On the one hand, we require the source term satisfy

$$
-\cos (\theta+\xi) \frac{\partial f_{ \pm, 0}}{\partial \eta_{ \pm}} \in L^{\infty}([0, \infty) \times[-\pi, \pi) \times[-\pi, \pi))
$$


to get a solution $Z_{ \pm} \in L^{\infty}([0, \infty) \times[-\pi, \pi) \times[-\pi, \pi))$ since we assume that $\partial_{\theta} g_{ \pm} \in L^{\infty}\left(\Gamma^{-}\right)$.

On the other hand, as be shown by the Appendix of [13], it holds that $\partial_{\eta} f_{ \pm, 0} \notin L^{\infty}([0, \infty) \times[-\pi, \pi) \times$ $[-\pi, \pi))$ for some specific boundary condition $g_{ \pm}$. Due to the intrinsic singularity for (2.28), the construction in [1] breaks down.

In fact, in general geometry domain with curved boundary, we need to control the normal derivative of the boundary layer solution for the Milne expansion. It is the main reason that we consider the following $\epsilon$-Milne expansion with geometric correction.

2.3. $\epsilon$-Milne Expansion with Geometric Correction. Our main goal is to overcome the difficulty in estimating

$$
\cos (\theta+\xi) \frac{\psi\left(\epsilon \eta_{ \pm}\right)}{R_{ \pm} \mp \epsilon \eta_{ \pm}} \frac{\partial \mathscr{U}_{ \pm, k}}{\partial \theta} .
$$

We introduce one more substitution to decompose the term (2.39). Now, we give the solution expansion in the following steps.

Substitution 1: Define the interior expansion as follows:

$$
U^{\epsilon}(\vec{x}, \vec{w}) \sim \sum_{k=0}^{\infty} \epsilon^{k} U_{k}^{\epsilon}(\vec{x}, \vec{w})
$$

where $U_{k}^{\epsilon}$ satisfies the same equations as $U_{k}$ in (2.9) and (2.10). Here, to highlight its dependence on $\epsilon$ via the $\epsilon$-Milne problem and boundary data, we add the superscript $\epsilon$.

Substitution 2: We make the rotation substitution for $u^{\epsilon}\left(\eta_{ \pm}, \theta, \xi\right) \rightarrow u^{\epsilon}\left(\eta_{ \pm}, \theta, \phi\right)$ with $\left(\eta_{ \pm}, \theta, \phi\right) \in\left[0,\left(R_{+}-\right.\right.$ $\left.\left.R_{-}\right) / \epsilon\right] \times[-\pi, \pi) \times[-\pi, \pi)$ as

$$
\left\{\begin{aligned}
\eta_{ \pm} & =\eta_{ \pm} \\
\theta & =\theta \\
\phi & =\theta+\xi
\end{aligned}\right.
$$

and transform the equation (1.1) into

$$
\left\{\begin{array}{l} 
\pm \sin \phi \frac{\partial u^{\epsilon}}{\partial \eta_{ \pm}}-\frac{\epsilon}{R_{ \pm} \mp \epsilon \eta_{ \pm}} \cos \phi\left(\frac{\partial u^{\epsilon}}{\partial \phi}+\frac{\partial u^{\epsilon}}{\partial \theta}\right)+u^{\epsilon}-\frac{1}{2 \pi} \int_{-\pi}^{\pi} u^{\epsilon} \mathrm{d} \phi=0 \\
u^{\epsilon}(0, \theta, \phi)=g_{ \pm}(\theta, \phi) \text { for } \pm \sin \phi>0 .
\end{array}\right.
$$

We define the $\epsilon$-Milne expansion with geometric correction of boundary layer as follows:

$$
\mathscr{U}_{ \pm}^{\epsilon}\left(\eta_{ \pm}, \theta, \phi\right) \sim \sum_{k=0}^{\infty} \epsilon^{k} \mathscr{U}_{ \pm, k}^{\epsilon}\left(\eta_{ \pm}, \theta, \phi\right),
$$

where $\mathscr{U}_{k}^{\epsilon}$ can be determined by comparing the order of $\epsilon$ via plugging (2.43) into the equation (2.42). Thus, in a neighborhood of the boundary, we have

$$
\begin{aligned}
& \pm \sin \phi \frac{\partial \mathscr{U}_{ \pm, 0}^{\epsilon}}{\partial \eta_{ \pm}}-\frac{\epsilon}{R_{ \pm} \mp \epsilon \eta_{ \pm}} \cos \phi \frac{\partial \mathscr{U}_{ \pm, 0}^{\epsilon}}{\partial \phi}+\mathscr{U}_{ \pm, 0}^{\epsilon}-\overline{\mathscr{U}}_{ \pm, 0}^{\epsilon}=0 \\
& \pm \sin \phi \frac{\partial \mathscr{U}_{ \pm, 1}^{\epsilon}}{\partial \eta_{ \pm}}-\frac{\epsilon}{R_{ \pm} \mp \epsilon \eta_{ \pm}} \cos \phi \frac{\partial \mathscr{U}_{ \pm, 1}^{\epsilon}}{\partial \phi}+\mathscr{U}_{ \pm, 1}^{\epsilon}-\overline{\mathscr{U}}_{ \pm, 1}^{\epsilon}=\frac{\cos \phi}{R_{ \pm} \mp \epsilon \eta_{ \pm}} \frac{\partial \mathscr{U}_{ \pm, 0}^{\epsilon}}{\partial \theta} \\
& \pm \sin \phi \frac{\partial \mathscr{U}_{ \pm, k}^{\epsilon}}{\partial \eta_{ \pm}}-\frac{\epsilon}{R_{ \pm} \mp \epsilon \eta_{ \pm}} \cos \phi \frac{\partial \mathscr{U}_{ \pm, k}^{\epsilon}}{\partial \phi}+\mathscr{U}_{ \pm, k}^{\epsilon}-\overline{\mathscr{U}}_{ \pm, k}^{\epsilon}=\frac{\cos \phi}{R_{ \pm} \mp \epsilon \eta_{ \pm}} \frac{\partial \mathscr{U}_{ \pm, k-1}^{\epsilon}}{\partial \theta} .
\end{aligned}
$$

where

$$
\overline{\mathscr{U}}_{ \pm, k}^{\epsilon}\left(\eta_{ \pm}, \theta\right)=\frac{1}{2 \pi} \int_{-\pi}^{\pi} \mathscr{U}_{ \pm, k}^{\epsilon}\left(\eta_{ \pm}, \theta, \phi\right) \mathrm{d} \phi
$$


Here the most important idea is to include the singular term

$$
-\frac{\epsilon}{R_{ \pm} \mp \epsilon \eta_{ \pm}} \cos \phi \frac{\partial u^{\epsilon}}{\partial \phi}
$$

in the Milne problem. It is notable that the solution $\mathscr{U}_{ \pm, k}^{\epsilon}$ depends on $\epsilon$.

Substitution 3: Similar to the classical expansion, we first consider the boundary condition expansion

$$
\begin{aligned}
U_{0}^{\epsilon}+\mathscr{U}_{ \pm, 0}^{\epsilon} & =g_{ \pm}, \\
U_{1}^{\epsilon}+\mathscr{U}_{ \pm, 1}^{\epsilon} & =0, \\
\ldots & \\
U_{k}^{\epsilon}+\mathscr{U}_{ \pm, k}^{\epsilon} & =0 .
\end{aligned}
$$

The construction of $U_{k}^{\epsilon}$ and $\mathscr{U}_{k}^{\epsilon}$ are as follows:

Step 1: Construction of $\mathscr{U}_{ \pm, 0}^{\epsilon}$ and $U_{0}^{\epsilon}$.

We refer to the cut-off function $\psi$ and $\psi_{0}$ as (2.26) and (2.27), and define the force as

$$
F_{ \pm}\left(\epsilon ; \eta_{ \pm}\right)=-\frac{\epsilon \psi\left(\epsilon \eta_{ \pm}\right)}{R_{ \pm} \mp \epsilon \eta_{ \pm}}
$$

The zeroth order boundary layer solutions is defined as

$$
\left\{\begin{array}{c}
\mathscr{U}_{ \pm, 0}^{\epsilon}\left(\eta_{ \pm}, \theta, \phi\right)=\psi_{0}\left(\epsilon \eta_{ \pm}\right)\left(f_{ \pm, 0}^{\epsilon}\left(\eta_{ \pm}, \theta, \phi\right)-f_{ \pm, 0}^{\epsilon}(\infty, \theta)\right), \\
\pm \sin \phi \frac{\partial f_{ \pm, 0}^{\epsilon}}{\partial \eta_{ \pm}}+F_{ \pm}\left(\epsilon ; \eta_{ \pm}\right) \cos \phi \frac{\partial f_{ \pm, 0}^{\epsilon}}{\partial \phi}+f_{ \pm, 0}^{\epsilon}-\bar{f}_{ \pm, 0}^{\epsilon}=0, \\
f_{ \pm, 0}^{\epsilon}(0, \theta, \phi)=g_{ \pm}(\theta, \phi) \text { for } \pm \sin \phi>0, \\
\lim _{\eta_{ \pm} \rightarrow \infty} f_{ \pm, 0}^{\epsilon}\left(\eta_{ \pm}, \theta, \phi\right)=f_{ \pm, 0}^{\epsilon}(\infty, \theta) .
\end{array}\right.
$$

In contrast to the classical Milne problem (2.28), the key advantage is, due to the geometry, $\frac{\partial F_{ \pm}\left(\epsilon ; \eta_{ \pm}\right)}{\partial \theta}=0$, such that (2.53) is invariant in $\theta$.

With the asymptotic behavior of the boundary layer solution in hand, we define the zeroth order interior solution $U_{0}^{\epsilon}(\vec{x})$ as

$$
\left\{\begin{aligned}
U_{0}^{\epsilon} & =\bar{U}_{0}^{\epsilon}, \\
\Delta_{x} \bar{U}_{0}^{\epsilon} & =0 \text { in } \Omega, \\
\bar{U}_{0}^{\epsilon} & =f_{ \pm, 0}^{\epsilon}(\infty, \theta) \text { on } \partial \Omega_{ \pm} .
\end{aligned}\right.
$$

Notice that the asymptotic state depends on $\epsilon$, then the interior solution depends on $\epsilon$ too.

Step 2: Estimates of $\frac{\partial \mathscr{U}_{ \pm, 0}^{\epsilon}}{\partial \theta}$.

By Theorem 3.13, we can easily see $f_{ \pm, 0}^{\epsilon}$ are well-defined in $L^{\infty}\left(\Omega \times \mathcal{S}^{1}\right)$ and approach to $f_{ \pm, 0}^{\epsilon}(\infty)$ exponentially fast as $\eta_{ \pm} \rightarrow \infty$. Then we can derive $Z_{ \pm}=\partial_{\theta}\left(f_{ \pm, 0}^{\epsilon}-f_{ \pm, 0}^{\epsilon}(\infty)\right)$ also satisfies the same type of $\epsilon$-Milne problem

$$
\left\{\begin{array}{c} 
\pm \sin \phi \frac{\partial Z_{ \pm}}{\partial \eta_{ \pm}}+F(\epsilon ; \eta) \cos \phi \frac{\partial Z_{ \pm}}{\partial \phi}+Z_{ \pm}-\bar{Z}_{ \pm}=0, \\
Z_{ \pm}(0, \theta, \phi)=\frac{\partial g_{ \pm}}{\partial \theta}-\frac{\partial f_{ \pm, 0}^{\epsilon}(\infty)}{\partial \theta} \text { for } \pm \sin \phi>0, \\
\lim _{\eta_{ \pm} \rightarrow \infty} Z_{ \pm}\left(\eta_{ \pm}, \phi\right)=C .
\end{array}\right.
$$

By Theorem 3.13, we can see $Z_{ \pm} \rightarrow C$ exponentially fast as $\eta_{ \pm} \rightarrow \infty$. It is natural to obtain this constant $C$ must be zero. Hence, if $g_{ \pm} \in C^{r}\left(\Gamma^{-}\right)$, it is obvious to check $f_{ \pm, 0}^{\epsilon}(\infty) \in C^{r}(\partial \Omega)$. By the standard elliptic estimate in (2.54), there exists a unique solution $\bar{U}_{ \pm, 0}^{\epsilon} \in W^{r, p}(\Omega)$ for arbitrary $p \geq 2$ satisfying

$$
\left\|\bar{U}_{ \pm, 0}^{\epsilon}\right\|_{W^{r, p}(\Omega)} \leq C(\Omega)\left\|f_{ \pm, 0}^{\epsilon}(\infty)\right\|_{W^{r-1 / p, p}(\partial \Omega)},
$$

which implies $\nabla_{x} \bar{U}_{0}^{\epsilon} \in W^{r-1, p}(\Omega), \nabla_{x} \bar{U}_{0}^{\epsilon} \in W^{r-1-1 / p, p}(\partial \Omega)$ and $\bar{U}_{0}^{\epsilon} \in C^{r-1,1-2 / p}(\Omega)$. 
Step 3: Construction of $\mathscr{U}_{ \pm, 1}^{\epsilon}$ and $U_{1}^{\epsilon}$.

The first order boundary layer solution is defined as

$$
\left\{\begin{array}{c}
\mathscr{U}_{ \pm, 1}^{\epsilon}\left(\eta_{ \pm}, \theta, \phi\right)=\psi_{0}\left(\epsilon \eta_{ \pm}\right)\left(f_{ \pm, 1}^{\epsilon}\left(\eta_{ \pm}, \theta, \phi\right)-f_{ \pm, 1}^{\epsilon}(\infty, \theta)\right) \\
\pm \sin \phi \frac{\partial f_{ \pm, 1}^{\epsilon}}{\partial \eta_{ \pm}}+F_{ \pm}\left(\epsilon ; \eta_{ \pm}\right) \cos \phi \frac{\partial f_{ \pm, 1}^{\epsilon}}{\partial \phi}+f_{ \pm, 1}^{\epsilon}-\bar{f}_{ \pm, 1}^{\epsilon}=\frac{\psi\left(\epsilon \eta_{ \pm}\right)}{R_{ \pm} \mp \epsilon \eta_{ \pm}} \cos \phi \frac{\partial \mathscr{U}_{ \pm, 0}^{\epsilon}}{\partial \theta} \\
f_{ \pm, 1}^{\epsilon}(0, \theta, \phi)=\vec{w} \cdot \nabla_{x} U_{0}^{\epsilon}\left(\vec{x}_{ \pm}, \vec{w}\right) \text { for } \quad \pm \sin \phi>0 \\
\lim _{\eta_{ \pm} \rightarrow \infty} f_{ \pm, 1}^{\epsilon}\left(\eta_{ \pm}, \theta, \phi\right)=f_{ \pm, 1}^{\epsilon}(\infty, \theta) .
\end{array}\right.
$$

Then, we define the first order interior solution $U_{1}^{\epsilon}(\vec{x})$ as

$$
\left\{\begin{aligned}
U_{1}^{\epsilon} & =\bar{U}_{1}^{\epsilon}-\vec{w} \cdot \nabla_{x} U_{0}^{\epsilon}, \\
\Delta_{x} \bar{U}_{1}^{\epsilon} & =0 \text { in } \Omega, \\
\bar{U}_{1}^{\epsilon} & =f_{ \pm, 1}^{\epsilon}(\infty, \theta) \text { on } \partial \Omega_{ \pm} .
\end{aligned}\right.
$$

Step 4: Estimates of $\frac{\partial \mathscr{U}_{ \pm, 1}^{\epsilon}}{\partial \theta}$.

By Theorem 3.13 we can easily see $f_{ \pm, 1}^{\epsilon}$ is well-defined in $L^{\infty}\left(\Omega \times \mathcal{S}^{1}\right)$ and approaches $f_{ \pm, 1}^{\epsilon}(\infty)$ exponentially fast as $\eta_{ \pm} \rightarrow \infty$. Also, since $\nabla_{x} U_{0}^{\epsilon} \in W^{r-1-1 / p, p}(\partial \Omega), \partial_{\theta} f_{ \pm, 1}^{\epsilon}$ is well-defined and decays exponentially fast. Hence, $f_{ \pm, 1}^{\epsilon}(\infty, \theta) \in W^{r-1-1 / p, p}(\partial \Omega)$. By the standard elliptic estimate in (2.58), there exists a unique solution $\bar{U}_{ \pm, 1}^{\epsilon} \in W^{r-1, p}(\Omega)$ and satisfies

$$
\left\|\bar{U}_{ \pm, 1}^{\epsilon}\right\|_{W^{r-1, p}(\Omega)} \leq C(\Omega)\left\|f_{ \pm, 1}^{\epsilon}(\infty)\right\|_{W^{r-1-1 / p, p}(\partial \Omega)}
$$

which implies $\nabla_{x} \bar{U}_{1}^{\epsilon} \in W^{r-2, p}(\Omega), \nabla_{x} \bar{U}_{1}^{\epsilon} \in W^{r-2-1 / p, p}(\partial \Omega)$ and $\bar{U}_{1}^{\epsilon} \in C^{r-2,1-2 / p}(\Omega)$.

Step 5: Generalization to arbitrary $k$.

In a similar fashion, as long as $g$ is sufficiently smooth, above process can be continued. We construct the $k^{t h}$ order boundary layer solution as

$$
\left\{\begin{array}{c}
\mathscr{U}_{ \pm, k}^{\epsilon}\left(\eta_{ \pm}, \theta, \phi\right)=\psi_{0}\left(\epsilon \eta_{ \pm}\right)\left(f_{ \pm, k}^{\epsilon}\left(\eta_{ \pm}, \theta, \phi\right)-f_{ \pm, k}^{\epsilon}(\infty, \theta)\right) \\
\pm \sin \phi \frac{\partial f_{ \pm, k}^{\epsilon}}{\partial \eta_{ \pm}}+F_{ \pm}\left(\epsilon ; \eta_{ \pm}\right) \cos \phi \frac{\partial f_{ \pm, k}^{\epsilon}}{\partial \phi}+f_{ \pm, k}^{\epsilon}-\bar{f}_{ \pm, k}^{\epsilon}=\frac{\psi\left(\epsilon \eta_{ \pm}\right)}{R_{ \pm} \mp \epsilon \eta} \cos \phi \frac{\partial \mathscr{U}_{ \pm, k-1}^{\epsilon}}{\partial \theta} \\
f_{ \pm, k}^{\epsilon}(0, \theta, \phi)=\vec{w} \cdot \nabla_{x} U_{k-1}^{\epsilon}\left(\vec{x}_{ \pm}, \vec{w}\right) \text { for } \pm \sin \phi>0 \\
\lim _{\eta_{ \pm} \rightarrow \infty} f_{ \pm, k}^{\epsilon}\left(\eta_{ \pm}, \theta, \phi\right)=f_{ \pm, k}^{\epsilon}(\infty, \theta)
\end{array}\right.
$$

Then we define the $k^{\text {th }}$ order interior solution as

$$
\left\{\begin{aligned}
U_{k}^{\epsilon} & =\bar{U}_{k}^{\epsilon}-\vec{w} \cdot \nabla_{x} U_{k-1}^{\epsilon}, \\
\Delta_{x} \bar{U}_{k}^{\epsilon} & =0 \text { in } \Omega, \\
\bar{U}_{k}^{\epsilon} & =f_{ \pm, k}^{\epsilon}(\infty, \theta) \text { on } \partial \Omega .
\end{aligned}\right.
$$

For $g_{ \pm} \in C^{k+1}\left(\Gamma^{-}\right)$, the interior solution and boundary layer solution can be well-defined up to $k^{\text {th }}$ order, i.e. up to $U_{k}^{\epsilon}$ and $\mathscr{U}_{ \pm, k}^{\epsilon}$.

\section{3. $\epsilon$-Milne Problem with Geometric Correction}

We consider the $\epsilon$-Milne problem for $f_{ \pm}^{\epsilon}\left(\eta_{ \pm}, \theta, \phi\right)$ in the domain $\left(\eta_{ \pm}, \theta, \phi\right) \in[0, \infty) \times[-\pi, \pi) \times[-\pi, \pi)$

$$
\left\{\begin{aligned}
\pm \sin \phi \frac{\partial f_{ \pm}^{\epsilon}}{\partial \eta_{ \pm}}+F_{ \pm}\left(\epsilon ; \eta_{ \pm}\right) \cos \phi \frac{\partial f_{ \pm}^{\epsilon}}{\partial \phi}+f_{ \pm}^{\epsilon}-\bar{f}_{ \pm}^{\epsilon} & =S_{ \pm}^{\epsilon}\left(\eta_{ \pm}, \theta, \phi\right), \\
f_{ \pm}^{\epsilon}(0, \theta, \phi) & =h_{ \pm}^{\epsilon}(\theta, \phi) \text { for } \pm \sin \phi>0, \\
\lim _{\eta_{ \pm} \rightarrow \infty} f_{ \pm}^{\epsilon}\left(\eta_{ \pm}, \theta, \phi\right) & =f_{ \pm, \infty}^{\epsilon}(\theta),
\end{aligned}\right.
$$


where

$$
\begin{gathered}
\bar{f}_{ \pm}^{\epsilon}\left(\eta_{ \pm}, \theta\right)=\frac{1}{2 \pi} \int_{-\pi}^{\pi} f_{ \pm}^{\epsilon}\left(\eta_{ \pm}, \theta, \phi\right) \mathrm{d} \phi \\
F_{ \pm}\left(\epsilon ; \eta_{ \pm}\right)=-\frac{\epsilon}{R_{ \pm} \mp \epsilon \eta_{ \pm}}, \\
\left|h_{ \pm}^{\epsilon}(\theta, \phi)\right| \leq M
\end{gathered}
$$

and

$$
\left|S_{ \pm}^{\epsilon}\left(\eta_{ \pm}, \theta, \phi\right)\right| \leq M e^{-K \eta_{ \pm}}
$$

for $M>0$ and $K>0$ uniform in $\epsilon$ and $\theta$.

The well-posedness and decay of $f_{+}$has been extensively studied in [13, Section 4], so in this section, we will focus on the following:

$$
\left\{\begin{aligned}
-\sin \phi \frac{\partial f_{-}^{\epsilon}}{\partial \eta_{-}}+F_{-}\left(\epsilon ; \eta_{-}\right) \cos \phi \frac{\partial f_{-}^{\epsilon}}{\partial \phi}+f_{-}^{\epsilon}-\bar{f}_{-}^{\epsilon} & =S_{-}^{\epsilon}\left(\eta_{-}, \theta, \phi\right) \\
f_{-}^{\epsilon}(0, \theta, \phi) & =h_{-}^{\epsilon}(\theta, \phi) \text { for }-\sin \phi>0 \\
\lim _{\eta_{-} \rightarrow \infty} f_{-}^{\epsilon}\left(\eta_{-}, \theta, \phi\right) & =f_{-, \infty}^{\epsilon}(\theta)
\end{aligned}\right.
$$

Introducing the sign substitution $\phi_{-}=-\phi$, we have

$$
\left\{\begin{aligned}
\sin \phi_{-} \frac{\partial f_{-}^{\epsilon}}{\partial \eta_{-}}-F_{-}\left(\epsilon ; \eta_{-}\right) \cos \phi_{-} \frac{\partial f_{-}^{\epsilon}}{\partial \phi_{-}}+f_{-}^{\epsilon}-\bar{f}_{-}^{\epsilon} & =S_{-}^{\epsilon}\left(\eta_{-}, \theta, \phi_{-}\right), \\
f_{-}^{\epsilon}\left(0, \theta, \phi_{-}\right) & =h_{-}^{\epsilon}\left(\theta, \phi_{-}\right) \text {for } \sin \phi_{-}>0 \\
\lim _{\eta_{-} \rightarrow \infty} f_{-}^{\epsilon}\left(\eta_{-}, \theta, \phi_{-}\right) & =f_{-, \infty}^{\epsilon}(\theta),
\end{aligned}\right.
$$

No abusing of the notation, we temporarily ignore the subscript - , superscript $\epsilon$, and the dependence on $\theta$ to have

$$
\left\{\begin{aligned}
\sin \phi \frac{\partial f}{\partial \eta}-F(\eta) \cos \phi \frac{\partial f}{\partial \phi}+f-\bar{f} & =S(\eta, \phi) \\
f(0, \phi) & =h(\phi) \text { for } \sin \phi>0 \\
\lim _{\eta \rightarrow \infty} f(\eta, \phi) & =f_{\infty},
\end{aligned}\right.
$$

where

$$
\begin{gathered}
\bar{f}(\eta)=\frac{1}{2 \pi} \int_{-\pi}^{\pi} f(\eta, \phi) \mathrm{d} \phi \\
F(\eta)=-\frac{\epsilon \psi(\epsilon \eta)}{R+\epsilon \eta} \\
|h(\phi)| \leq M
\end{gathered}
$$

and

$$
|S(\eta, \phi)| \leq M e^{-K \eta}
$$

for $M>0$ and $K>0$. We may further define a potential function $V(\eta)$ satisfying

$$
V(\epsilon, 0)=0, \quad \frac{\mathrm{d} V(\epsilon, \eta)}{\mathrm{d} \eta}=-F(\epsilon, \eta) \geq 0 .
$$

Since $V(\epsilon, \eta)$ is monotonically increasing w.r.t $\eta$ and $\operatorname{supp}[\psi] \subset[0,3 / 4]$, we can derive

$$
0 \leq V(\epsilon, \eta) \leq \ln 4,1 \leq \exp (V(\epsilon, \eta)) \leq 4 \text { for all } \eta \in[0, \infty] .
$$


In this section, we introduce some special notation to describe the norms in the space $(\eta, \phi) \in[0, \infty) \times$ $[-\pi, \pi)$. Define the $L^{2}$ norm as follows:

$$
\begin{aligned}
\|f(\eta, \cdot)\|_{L^{2}} & =\left(\int_{-\pi}^{\pi}|f(\eta, \phi)|^{2} \mathrm{~d} \phi\right)^{1 / 2} \\
\|f\|_{L^{2} L^{2}} & =\left(\int_{0}^{\infty} \int_{-\pi}^{\pi}|f(\eta, \phi)|^{2} \mathrm{~d} \phi \mathrm{d} \eta\right)^{1 / 2} .
\end{aligned}
$$

Define the inner product in $\phi$ space

$$
\langle f, g\rangle_{\phi}(\eta)=\int_{-\pi}^{\pi} f(\eta, \phi) g(\eta, \phi) \mathrm{d} \phi
$$

Define the $L^{\infty}$ norm as follows:

$$
\begin{aligned}
\|f(\eta)\|_{L^{\infty}} & =\sup _{\phi \in[-\pi, \pi)}|f(\eta, \phi)|, \\
\|f\|_{L^{\infty} L^{\infty}} & =\sup _{(\eta, \phi) \in[0, \infty) \times[-\pi, \pi)}|f(\eta, \phi)|, \\
\|f\|_{L^{\infty} L^{2}} & =\sup _{\eta \in[0, \infty)}\left(\int_{-\pi}^{\pi}|f(\eta, \phi)|^{2} \mathrm{~d} \phi\right)^{1 / 2} .
\end{aligned}
$$

Since the boundary data $h(\phi)$ is only defined on $\sin \phi>0$, we naturally extend above definitions on this half-domain as follows:

$$
\begin{aligned}
\|h\|_{L^{2}} & =\left(\int_{\sin \phi>0}|h(\phi)|^{2} \mathrm{~d} \phi\right)^{1 / 2} \\
\|h\|_{L^{\infty}} & =\sup _{\sin \phi>0}|h(\phi)| .
\end{aligned}
$$

Lemma 3.1. We have

$$
\begin{aligned}
\|h\|_{L^{2}} & \leq C\|h\|_{L^{\infty}} \leq C M \\
\|S\|_{L^{2} L^{2}} & \leq C \frac{M}{K} \\
\|S\|_{L^{\infty} L^{2}} & \leq C\|S\|_{L^{\infty} L^{\infty}} \leq C M
\end{aligned}
$$

Proof. They can be verified via direct computation, so we omit the proofs here.

\section{1. $L^{2}$ Estimates.}

3.1.1. Finite Slab with $\bar{S}=0$. Consider the $\epsilon$-Milne problem for $f^{L}(\eta, \phi)$ in a finite slab $(\eta, \phi) \in[0, L] \times$ $[-\pi, \pi)$

$$
\left\{\begin{aligned}
\sin \phi \frac{\partial f^{L}}{\partial \eta}-F(\eta) \cos \phi \frac{\partial f^{L}}{\partial \phi}+f^{L}-\bar{f}^{L} & =S(\eta, \phi) \\
f^{L}(0, \phi) & =h(\phi) \text { for } \sin \phi>0 \\
f^{L}(L, \phi) & =f^{L}(L, R \phi),
\end{aligned}\right.
$$

where $R \phi=-\phi$ and $S$ satisfies $\bar{S}(\eta)=0$ for any $\eta$. We may decompose the solution

$$
f^{L}(\eta, \phi)=q_{f}^{L}(\eta)+r_{f}^{L}(\eta, \phi),
$$

where the hydrodynamical part $q_{f}^{L}$ of $f^{L}$, and the microscopic part $r_{f}^{L}$ is the orthogonal complement, i.e.

$$
q_{f}^{L}(\eta)=\bar{f}^{L}=\frac{1}{2 \pi} \int_{-\pi}^{\pi} f^{L}(\eta, \phi) \mathrm{d} \phi, \quad r_{f}^{L}(\eta, \phi)=f^{L}(\eta, \phi)-q_{f}^{L}(\eta) .
$$

In the following, we simply write $f^{L}=q^{L}+r^{L}$ without any confusion. 
Lemma 3.2. Assume $\bar{S}(\eta)=0(\eta \in[0, L])$ and $S(\eta, \phi)$ satisfy (3.11) and (3.12). Then there exists a solution $f(\eta, \phi) \in L^{2}([0, L] \times[0,2 \pi])$ to the finite slab problem (3.26) such that

$$
\begin{aligned}
\int_{0}^{L}\left\|r^{L}(\eta, \cdot)\right\|_{L^{2}}^{2} \mathrm{~d} \eta & \leq C\left(M+\frac{M}{K}\right)^{2}<\infty, \\
\left|q^{L}(\eta)\right| & \leq C\left(1+M+\frac{M}{K}\right)\left(1+\eta^{1 / 2}\right)+\left\|r^{L}(\eta, \cdot)\right\|_{L^{2}}, \\
\left\langle\sin \phi, r^{L}\right\rangle_{\phi}(\eta) & =0,
\end{aligned}
$$

for arbitrary $\eta \in[0, L]$.

Proof. We divide the proof into several steps: we firstly establish the solution of the penalty problem. Secondly, the uniform estimates of the solution will be obtained. Then, by passing the limit, we achieve a solution of (3.26) which satisfies (3.29) and (3.30). At last, the orthogonal property (3.31) follows easily.

Step 1: Existence of the solution for the penalty problem

Firstly, we consider the existence of the solution for the penalized $\epsilon$-Milne equation with $\lambda>0$

$$
\left\{\begin{aligned}
\lambda f_{\lambda}+\sin \phi \frac{\partial f_{\lambda}}{\partial \eta}-F(\eta) \cos \phi \frac{\partial f_{\lambda}}{\partial \phi}+f_{\lambda} & =S(\eta, \phi), \\
f_{\lambda}(0, \phi) & =h(\phi) \text { for } \sin \phi>0, \\
f_{\lambda}(L, \phi) & =f_{\lambda}(L, R \phi) .
\end{aligned}\right.
$$

Here $\|S\|_{L^{2} L^{2}}<\infty$ and $\|h\|_{L^{2}}<\infty$.

For the existence of the solution for (3.32), we also construct the iterative sequence. Define $f_{0}^{L}=0$ and $\left\{f_{m}^{L}\right\}_{m=1}^{\infty}$ are the solution of the following problem

$$
\left\{\begin{aligned}
\lambda f_{m}^{L}+\sin \phi \frac{\partial f_{m}^{L}}{\partial \eta}-F(\eta) \cos \phi \frac{\partial f_{m}^{L}}{\partial \phi}+f_{m}^{L}-\bar{f}_{m-1}^{L} & =S(\eta, \phi) \\
f_{m}^{L}(0, \phi) & =h(\phi) \text { for } \sin \phi>0 \\
f_{m}^{L}(L, \phi) & =f_{m}^{L}(L, R \phi) .
\end{aligned}\right.
$$

For $m=1$, we have $\bar{f}_{0}^{L}=0$. Multiplying $f_{1}^{L}$ on both sides of (3.33) and integrating over $\phi \in[-\pi, \pi]$, we obtain

$$
\frac{1}{2} \frac{\mathrm{d}}{\mathrm{d} \eta}\left\langle f_{1}^{L}, f_{1}^{L} \sin \phi\right\rangle_{\phi}-\frac{1}{2} F(\eta)\left\langle f_{1}^{L}, f_{1}^{L} \sin \phi\right\rangle_{\phi}+(1+\lambda)\left\|f_{1}^{L}\right\|_{L^{2}}^{2}=\left\langle S, f_{1}^{L}\right\rangle_{\phi}
$$

It leads to

$$
\begin{aligned}
& \exp [V(L)]\left\langle f_{1}^{L}, f_{1}^{L} \sin \phi\right\rangle_{\phi}(L)+(1+\lambda) \int_{0}^{L} \exp [V(y)]\left\|f_{1}^{L}(y, \cdot)\right\|_{L^{2}}^{2} \mathrm{~d} y \\
= & \left\langle f_{1}^{L}, f_{1}^{L} \sin \phi\right\rangle_{\phi}(0)+\int_{0}^{L} \exp [V(y)]\left\langle S, f_{1}^{L}\right\rangle_{\phi} \mathrm{d} y \\
\leq & \|h\|_{L^{2}}+\int_{0}^{L} \exp [V(y)]\left[\frac{1}{2}\|S(y, \cdot)\|_{L^{2}}^{2}+\frac{1}{2}\left\|f_{1}^{L}(y, \cdot)\right\|_{L^{2}}^{2}\right] \mathrm{d} y .
\end{aligned}
$$

where $V$ is the potential function which is defined in (3.13). It is easy to show that $\exp [V(L)]\left\langle f_{1}^{L}, f_{1}^{L} \sin \phi\right\rangle_{\phi}(L)=$ 0 because the specular refection condition at $\eta=L$. From (3.14) and the above inequality, one obtains that

$$
\int_{0}^{L}\left\|f_{1}^{L}(y, \cdot)\right\|_{L^{2}}^{2} \mathrm{~d} y \leq 8\left(\|h\|_{L^{2}}^{2}+\|S\|_{L^{2} L^{2}}^{2}\right) .
$$

For $m \geq 1$, we set $g_{m}^{L}=f_{m}^{L}-f_{m-1}^{L}$. It satisfies

$$
\left\{\begin{aligned}
\lambda g_{m}^{L}+\sin \phi \frac{\partial g_{m}^{L}}{\partial \eta}-F(\eta) \cos \phi \frac{\partial g_{m}^{L}}{\partial \phi}+g_{m}^{L}-\bar{g}_{m-1}^{L} & =0 \\
g_{m}^{L}(0, \phi) & =0 \text { for } \sin \phi>0, \\
g_{m}^{L}(L, \phi) & =g_{m}^{L}(L, R \phi) .
\end{aligned}\right.
$$


By a similar argument, we can derive that

$$
\begin{aligned}
& \exp [V(L)]\left\langle g_{m}^{L}, g_{m}^{L} \sin \phi\right\rangle_{\phi}(L)+\int_{0}^{L} \exp [V(y)]\left((1+\lambda)\left\|g_{m}^{L}(y, \cdot)\right\|_{L^{2}}^{2}+\left\langle g_{m}^{L}, \bar{g}_{m-1}^{L}\right\rangle_{\phi}(y)\right) \mathrm{d} y \\
& \leq\left\langle g_{m}^{L}, g_{m}^{L} \sin \phi\right\rangle_{\phi}(0)
\end{aligned}
$$

Due to the facts $\left\langle g_{m}^{L}, g_{m}^{L} \sin \phi\right\rangle_{\phi}(0) \leq 0, \exp [V(L)]\left\langle g_{m}^{L}, g_{m}^{L} \sin \phi\right\rangle_{\phi}(L)=0$, we have

$$
\begin{aligned}
\int_{0}^{L} \exp [V(y)]\left\|g_{m}^{L}(y, \cdot)\right\|_{L^{2}}^{2} \mathrm{~d} y & \leq \frac{1}{1+\lambda} \int_{0}^{L} \exp [V(y)]\left\langle g_{m}^{L}, \bar{g}_{m-1}^{L}\right\rangle_{\phi}(y) \mathrm{d} y \\
& \leq \frac{1}{2(1+\lambda)} \int_{0}^{L} \exp [V(y)]\left(\left\|g_{m}^{L}(y, \cdot)\right\|_{L^{2}}^{2}+\left\|g_{m-1}^{L}(y, \cdot)\right\|_{L^{2}}^{2}\right) \mathrm{d} y .
\end{aligned}
$$

It implies

$$
\int_{0}^{L} \exp [V(y)]\left\|g_{m}^{L}(y, \cdot)\right\|_{L^{2}}^{2} \mathrm{~d} y \leq \frac{1}{1+\lambda} \int_{0}^{L} \exp [V(y)]\left\|g_{m-1}^{L}(y, \cdot)\right\|_{L^{2}}^{2} \mathrm{~d} y .
$$

Since $\exp [V(y)] \geq 1$ for all $y \geq 0$, we know that $\left\{f_{m}^{L}\right\}_{m=1}^{\infty}$ is a contraction sequence for $\lambda>0$. Let $m \rightarrow \infty$, we obtain a solution $f_{\lambda}^{L}$ of (3.32). Moreover, it satisfies

$$
\left\|f_{\lambda}^{L}\right\|_{L^{2} L^{2}}^{2} \leq\left\|f_{1}^{L}\right\|_{L^{2} L^{2}}^{2}+\sum_{m=1}^{\infty}\left\|g_{m}^{L}\right\|_{L^{2} L^{2}}^{2} \leq \frac{8(1+\lambda)}{\lambda}\left(\|h\|_{L^{2}}^{2}+\|S\|_{L^{2} L^{2}}^{2}\right) .
$$

In the following, we prove the uniqueness of the solution for (3.32). Assume that there are two solutions $f_{1}^{L}$ and $f_{2}^{L}$ of (3.32). Define $f_{*}^{L}=f_{1}-f_{2}$, it satisfies

$$
\left\{\begin{aligned}
\lambda f_{*}^{L}+\sin \phi \frac{\partial f_{*}^{L}}{\partial \eta}-F(\eta) \cos \phi \frac{\partial f_{*}^{L}}{\partial \phi}+f_{*}^{L}-\bar{f}_{*}^{L} & =0 \\
f_{*}^{L}(0, \phi) & =0 \text { for } \sin \phi>0 \\
f_{*}^{L}(L, \phi) & =f_{*}^{L}(L, R \phi) .
\end{aligned}\right.
$$

Similarly, we have

$$
\left\|f_{*}^{L}\right\|_{L^{2} L^{2}}^{2} \leq \frac{1}{1+\lambda}\left\|\bar{f}_{*}^{L}\right\|_{L^{2} L^{2}}^{2} \leq \frac{1}{1+\lambda}\left\|f_{*}^{L}\right\|_{L^{2} L^{2}}^{2}
$$

which further implies $f_{*}^{L}=0$ when $\lambda>0$. Therefore, the solution to (3.32) is unique in $L^{2}([0, L] \times[-\pi, \pi)$ ).

Step 2: The uniform estimates of $r_{\lambda}^{L}$ and $q_{\lambda}^{L}$ w.r.t. $\lambda$.

Claim: We claim that $r_{\lambda}^{L}$ satisfies

$$
\int_{0}^{L}\left\|r_{\lambda}^{L}(\eta, \cdot)\right\|_{L^{2}}^{2} \mathrm{~d} \eta \leq 4\|h\|_{L^{2}}^{2}+8 \int_{0}^{L}\|S(\eta, \cdot)\|_{L^{2}}^{2} \mathrm{~d} \eta
$$

and, for any $0 \leq \eta \leq L, q_{\lambda}^{L}$ satisfies

$$
\left|q_{\lambda}^{L}(\eta)\right| \leq 16 \pi(1+\lambda+|F(\eta)|)\left(1+\eta^{1 / 2}\right)\left(\|h\|_{L^{2}}+\|S\|_{L^{2} L^{2}}\right)+\left\|r_{\lambda}^{L}(\eta, \cdot)\right\|_{L^{2}} .
$$

The proof of (3.44): The assumption $\bar{S}(\eta)=0$ leads to

$$
\left\langle S, f_{\lambda}^{L}\right\rangle_{\phi}(\eta)=\left\langle S, q_{\lambda}^{L}\right\rangle_{\phi}(\eta)+\left\langle S, r_{\lambda}^{L}\right\rangle_{\phi}(\eta)=\left\langle S, r_{\lambda}^{L}\right\rangle_{\phi}(\eta)
$$

Multiplying $f_{\lambda}^{L}$ on both sides of (3.32) and integrating over $\phi \in[-\pi, \pi)$, we get the energy estimate

$$
\begin{aligned}
\frac{1}{2} \frac{\mathrm{d}}{\mathrm{d} \eta}\left\langle f_{\lambda}^{L}, f_{\lambda}^{L} \sin \phi\right\rangle_{\phi}(\eta)-\frac{1}{2} F(\eta)\left\langle f_{\lambda}^{L}, f_{\lambda}^{L} \sin \phi\right\rangle_{\phi}(\eta) \\
=-\lambda\left\|f_{\lambda}^{L}(\eta, \cdot)\right\|_{L^{2}}^{2}-\left\|r_{\lambda}^{L}(\eta, \cdot)\right\|_{L^{2}}^{2}+\left\langle S, r_{\lambda}^{L}\right\rangle_{\phi}(\eta) .
\end{aligned}
$$

Define

$$
\alpha(\eta)=\frac{1}{2}\left\langle f_{\lambda}^{L}, f_{\lambda}^{L} \sin \phi\right\rangle_{\phi}(\eta)
$$


Then (3.47) can be rewritten as follows

$$
\frac{\mathrm{d} \alpha}{\mathrm{d} \eta}-F(\eta) \alpha(\eta)=-\lambda\left\|f_{\lambda}^{L}(\eta, \cdot)\right\|_{L^{2}}^{2}-\left\|r_{\lambda}^{L}(\eta, \cdot)\right\|_{L^{2}}^{2}+\left\langle S, r_{\lambda}^{L}\right\rangle_{\phi}(\eta) .
$$

The specular reflexive boundary $f_{\lambda}^{L}(L, \phi)=f_{\lambda}^{L}(L, R \phi)$ ensures $\alpha(L)=0$. We can integrate above on $[\eta, L]$ and $[0, \eta]$ respectively to obtain

$$
\begin{aligned}
& \exp [V(\eta)] \alpha(\eta)=\int_{\eta}^{L} \exp [V(y)]\left(\lambda\left\|f_{\lambda}^{L}(y, \cdot)\right\|_{L^{2}}^{2}+\left\|r_{\lambda}^{L}(y, \cdot)\right\|_{L^{2}}^{2}-\left\langle S, r_{\lambda}^{L}\right\rangle_{\phi}(y)\right) \mathrm{d} y \\
& \exp [V(\eta)] \alpha(\eta)=\alpha(0)+\int_{0}^{\eta} \exp [V(y)]\left(-\lambda\left\|f_{\lambda}^{L}(y, \cdot)\right\|_{L^{2}}^{2}-\left\|r_{\lambda}^{L}(y, \cdot)\right\|_{L^{2}}^{2}+\left\langle S, r_{\lambda}^{L}\right\rangle_{\phi}(y)\right) \mathrm{d} y
\end{aligned}
$$

Hence, based on (3.50), we have

$$
\alpha(\eta) \geq \int_{\eta}^{L} \exp [V(y)-V(\eta)]\left(-\left\langle S, r_{\lambda}^{L}\right\rangle_{\phi}(y)\right) \mathrm{d} y
$$

Also, 3.51) implies

$$
\begin{aligned}
\alpha(\eta) & \leq \alpha(0) \exp [-V(\eta)]+\int_{0}^{\eta} \exp [V(\eta)-V(y)]\left(\left\langle S, r_{\lambda}^{L}\right\rangle_{\phi}(y)\right) \mathrm{d} y \\
& \leq 2\|h\|_{L^{2}}^{2}+\int_{0}^{\eta} \exp [V(\eta)-V(y)]\left(\left\langle S, r_{\lambda}^{L}\right\rangle_{\phi}(y)\right) \mathrm{d} y
\end{aligned}
$$

due to the fact

$$
\alpha(0)=\frac{1}{2}\left\langle\sin \phi f_{\lambda}^{L}, f_{\lambda}^{L}\right\rangle_{\phi}(0) \leq \frac{1}{2}\left(\int_{\sin \phi>0} h^{2}(\phi) \sin \phi \mathrm{d} \phi\right) \leq \frac{1}{2}\|h\|_{L^{2}}^{2} .
$$

Then in 3.51) taking $\eta=L$, from $\alpha(L)=0$, we have

$$
\begin{aligned}
\int_{0}^{L} \exp [V(y)]\left\|r_{\lambda}^{L}(y, \cdot)\right\|_{L^{2}}^{2} \mathrm{~d} y & \leq \alpha(0)+\int_{0}^{L} \exp [V(y)]\left\langle S, r_{\lambda}^{L}\right\rangle_{\phi}(y) \mathrm{d} y \\
& \leq \frac{1}{2}\|h\|_{L^{2}}^{2}+\int_{0}^{L} \exp [V(y)]\left\langle S, r_{\lambda}^{L}\right\rangle_{\phi}(y) \mathrm{d} y .
\end{aligned}
$$

On the other hand, we can directly estimate as follows:

$$
\int_{0}^{L} \exp [V(y)]\left\|r_{\lambda}^{L}(y, \cdot)\right\|_{L^{2}}^{2} \mathrm{~d} y \geq \int_{0}^{L}\left\|r_{\lambda}^{L}(y, \cdot)\right\|_{L^{2}}^{2} \mathrm{~d} y .
$$

Combining (3.55) and (3.224) yields

$$
\int_{0}^{L}\left\|r_{\lambda}^{L}(\eta, \cdot)\right\|_{L^{2}}^{2} \mathrm{~d} \eta \leq\|h\|_{L^{2}}^{2}+4 \int_{0}^{L}\left|\left\langle S, r_{\lambda}^{L}\right\rangle_{\phi}(y)\right| \mathrm{d} y
$$

By Cauchy's inequality, we have

$$
\int_{0}^{L}\left|\left\langle S, r_{\lambda}^{L}\right\rangle_{\phi}(y)\right| \mathrm{d} y \leq \frac{1}{8} \int_{0}^{L}\left\|r_{\lambda}^{L}(\eta, \cdot)\right\|_{L^{2}}^{2} \mathrm{~d} \eta+2 \int_{0}^{L}\|S(\eta, \cdot)\|_{L^{2}}^{2} \mathrm{~d} \eta .
$$

Therefore, summarizing (3.178) and (3.180), we deduce (3.44).

The proof of (3.45): Multiplying $\sin \phi$ on both sides of (3.32) and integrating over $\phi \in[-\pi, \pi)$ lead to

$$
\begin{aligned}
\frac{\mathrm{d}}{\mathrm{d} \eta}\left\langle\sin ^{2} \phi, f_{\lambda}^{L}\right\rangle_{\phi}(\eta)= & -\lambda\left\langle\sin \phi, f_{\lambda}^{L}\right\rangle_{\phi}(\eta)-\left\langle\sin \phi, r_{\lambda}^{L}\right\rangle_{\phi}(\eta) \\
& +\frac{1}{2} F(\eta)\left\langle\sin (2 \phi), \frac{\partial f_{\lambda}^{L}}{\partial \phi}\right\rangle_{\phi}(\eta)+\langle\sin \phi, S\rangle_{\phi}(\eta) .
\end{aligned}
$$

It is nature that

$$
\left\langle\sin (\phi), q_{\lambda}^{L}\right\rangle_{\phi}=q_{\lambda}^{L}\langle\sin (\phi), 1\rangle=0,\left\langle\cos (2 \phi), q_{\lambda}^{L}\right\rangle=q_{\lambda}^{L}\langle\cos (2 \phi), 1\rangle_{\phi}=0
$$


We can further integrate by parts as follows:

$$
\frac{1}{2} F(\eta)\left\langle\sin (2 \phi), \frac{\partial f_{\lambda}^{L}}{\partial \phi}\right\rangle_{\phi}(\eta)=-F(\eta)\left\langle\cos (2 \phi), f_{\lambda}^{L}\right\rangle_{\phi}(\eta)=-F(\eta)\left\langle\cos (2 \phi), r_{\lambda}^{L}\right\rangle_{\phi}(\eta),
$$

to obtain

$$
\begin{aligned}
\frac{\mathrm{d}}{\mathrm{d} \eta}\left\langle\sin ^{2} \phi, f_{\lambda}^{L}\right\rangle_{\phi}(\eta)= & -\lambda\left\langle\sin \phi, r_{\lambda}^{L}\right\rangle_{\phi}(\eta)-\left\langle\sin \phi, r_{\lambda}^{L}\right\rangle_{\phi}(\eta) \\
& -F(\eta)\left\langle\cos (2 \phi), r_{\lambda}^{L}\right\rangle_{\phi}(\eta)+\langle\sin \phi, S\rangle_{\phi}(\eta) .
\end{aligned}
$$

Define

$$
\beta_{\lambda}^{L}(\eta)=\left\langle\sin ^{2} \phi, f_{\lambda}^{L}\right\rangle_{\phi}(\eta)
$$

and

$$
D_{\lambda}^{L}(\eta, \phi)=-\lambda\left\langle\sin \phi, f_{\lambda}^{L}\right\rangle_{\phi}(\eta)-\left\langle\sin \phi, r_{\lambda}^{L}\right\rangle_{\phi}(\eta)-F(\eta)\left\langle\cos (2 \phi), r_{\lambda}^{L}\right\rangle_{\phi}(\eta)+\langle\sin \phi, S\rangle_{\phi}(\eta)
$$

Since

$$
-\lambda\left\langle\sin \phi, f_{\lambda}^{L}\right\rangle_{\phi}(\eta)=-\lambda\left\langle\sin \phi, r_{\lambda}^{L}\right\rangle_{\phi}(\eta)-\lambda\left\langle\sin \phi, q_{\lambda}^{L}\right\rangle_{\phi}(\eta)=-\lambda\left\langle\sin \phi, r_{\lambda}^{L}\right\rangle_{\phi}(\eta) .
$$

we can further get

$$
D_{\lambda}^{L}(\eta)=-\lambda\left\langle\sin \phi, r_{\lambda}^{L}\right\rangle_{\phi}(\eta)-\left\langle\sin \phi, r_{\lambda}^{L}\right\rangle_{\phi}(\eta)-F(\eta)\left\langle\cos (2 \phi), r_{\lambda}^{L}\right\rangle_{\phi}(\eta)+\langle\sin \phi, S\rangle_{\phi}(\eta) .
$$

Then we can simplify (3.59) as follows:

$$
\frac{\mathrm{d} \beta_{\lambda}^{L}}{\mathrm{~d} \eta}=D_{\lambda}^{L}(\eta, \phi)
$$

We can integrate over $[0, \eta]$ in (3.67) to obtain

$$
\beta_{\lambda}^{L}(\eta)=\beta_{\lambda}^{L}(0)+\int_{0}^{\eta} D_{\lambda}^{L}(y) \mathrm{d} y .
$$

On the hand, one has

$$
\begin{aligned}
\beta_{\lambda}^{L}(0) & =\left\langle\sin ^{2} \phi, f_{\lambda}^{L}\right\rangle_{\phi}(0) \leq\left(\left\langle f_{\lambda}^{L}, f_{\lambda}^{L}|\sin \phi|\right\rangle_{\phi}(0)\right)^{1 / 2} \| \sin \phi||_{L^{3}}^{3 / 2} \\
& \leq \frac{16}{3}\left(\left\langle f_{\lambda}^{L}, f_{\lambda}^{L}|\sin \phi|\right\rangle_{\phi}(0)\right)^{1 / 2}
\end{aligned}
$$

Obviously, we have

$$
\left\langle f_{\lambda}^{L}, f_{\lambda}^{L}|\sin \phi|\right\rangle_{\phi}(0)=\int_{\sin \phi>0} h^{2}(\phi) \sin \phi \mathrm{d} \phi-\int_{\sin \phi<0}\left|f_{\lambda}^{L}(0, \phi)\right|^{2} \sin \phi \mathrm{d} \phi .
$$

However, based on the definition of $\alpha(\eta)$ and (3.52), we can obtain

$$
\begin{aligned}
2 \alpha(0) & =\int_{\sin \phi>0} h^{2}(\phi) \sin \phi \mathrm{d} \phi+\int_{\sin \phi<0}\left|f_{\lambda}^{L}(0, \phi)\right|^{2} \sin \phi \mathrm{d} \phi \\
& \geq 2 \int_{0}^{L} \exp [V(y)]\left(-\left\langle S, r_{\lambda}^{L}\right\rangle_{\phi}(y)\right) \mathrm{d} y \\
& \geq-\frac{1}{2} \int_{0}^{L}\left|\left\langle S, r_{\lambda}^{L}\right\rangle_{\phi}(y)\right| \mathrm{d} y .
\end{aligned}
$$

Hence, we can deduce

$$
\begin{aligned}
\left\langle f_{\lambda}^{L}, f_{\lambda}^{L}|\sin \phi|\right\rangle_{\phi}(0) & \leq 2 \int_{\sin \phi>0} h^{2}(\phi) \sin \phi \mathrm{d} \phi+\frac{1}{2} \int_{0}^{L}\left|\left\langle S, r_{\lambda}^{L}\right\rangle_{\phi}(y)\right| \mathrm{d} y \\
& \leq 2\|h\|_{L^{2}}^{2}+\frac{1}{4} \int_{0}^{L}\left\|r_{\lambda}^{L}(\eta, \cdot)\right\|_{L^{2}}^{2} \mathrm{~d} \eta+\frac{1}{4} \int_{0}^{L}\|S(\eta, \cdot)\|_{L^{2}}^{2} \mathrm{~d} \eta .
\end{aligned}
$$


From (3.44), we can deduce

$$
\begin{aligned}
\beta_{\lambda}^{L}(0)^{2} & \leq \frac{256}{9}\left\langle f_{\lambda}^{L}, f_{\lambda}^{L}|\sin \phi|\right\rangle_{\phi}(0) \\
& \leq 128\|h\|_{L^{2}}^{2}+16 \int_{0}^{L}\left\|r_{\lambda}^{L}(\eta, \cdot)\right\|_{L^{2}}^{2} \mathrm{~d} \eta+16 \int_{0}^{L}\|S(\eta, \cdot)\|_{L^{2}}^{2} \mathrm{~d} \eta \\
& \leq 196\|h\|_{L^{2}}^{2}+196 \int_{0}^{L}\|S(\eta, \cdot)\|_{L^{2}}^{2} \mathrm{~d} \eta
\end{aligned}
$$

On the other hand, since $D_{\lambda}^{L}$ depends on $r_{\lambda}^{L}$ and is independent of $q_{\lambda}^{L}$, we can directly estimate

$$
\left|D_{\lambda}^{L}(\eta)\right| \leq 2 \pi(1+\lambda+|F(\eta)|)\left\|r_{\lambda}^{L}(\eta, \cdot)\right\|_{L^{2}}+\|S(\eta, \cdot)\|_{L^{2}} .
$$

From (3.44), (3.68), (3.74) and (3.73), we have

$$
\begin{aligned}
\left|\beta_{\lambda}^{L}(\eta)\right| \leq & 14\left(\|h\|_{L^{2}}+\|S\|_{L^{2} L^{2}}\right)+2 \pi(1+\lambda+|F(\eta)|) \int_{0}^{\eta}\left\|r_{\lambda}^{L}(y, \cdot)\right\|_{L^{2}} \mathrm{~d} y+\int_{0}^{\eta}\|S(y, \cdot)\|_{L^{2}} \mathrm{~d} y \\
\leq & 14\left(\|h\|_{L^{2}}+\|S\|_{L^{2} L^{2}}\right)+2 \pi(1+\lambda+|F(\eta)|) \eta^{1 / 2}\left(\int_{0}^{\eta}\left\|r_{\lambda}^{L}(y, \cdot)\right\|_{L^{2}}^{2} \mathrm{~d} y\right)^{1 / 2} \\
& \quad+\eta^{1 / 2}\left(\int_{0}^{\eta}\|S(y, \cdot)\|_{L^{2}}^{2} \mathrm{~d} y\right)^{1 / 2} \\
\leq & 16 \pi(1+\lambda+|F(\eta)|)\left(1+\eta^{1 / 2}\right)\left(\|h\|_{L^{2}}+\|S\|_{L^{2} L^{2}}\right) .
\end{aligned}
$$

By (3.63) this implies

$$
\left|q_{\lambda}^{L}(\eta)\right| \leq \frac{1}{\pi}\left(\left|\beta_{\lambda}^{L}(\eta)\right|+\left\|\sin ^{2}(\cdot)\right\|_{L^{2}}\left\|r_{\lambda}^{L}(\eta, \cdot)\right\|_{L^{2}}\right)
$$

which completes the proof of (3.45).

Step 3: Passing to the limit $\lambda \rightarrow 0$

Since estimates (3.44) and (3.45) are uniform in $\lambda$, we can take weakly convergent subsequence $f_{\lambda}^{L} \rightarrow f^{L} \in$ $L^{2}([0, L] \times[-\pi, \pi))$ as $\lambda \rightarrow 0$. That is, there is a function $f^{L} \in L^{2}([0, L] \times(-\pi, \pi])$, which is the solution of (3.26) and satisfies the estimates (3.29) and (3.30).

Step 4: Orthogonality relation 3.31).

A direct integration over $\phi \in[-\pi, \pi)$ in (3.26) implies

$$
\frac{\mathrm{d}}{\mathrm{d} \eta}\left\langle\sin \phi, f^{L}\right\rangle_{\phi}(\eta)=F\left\langle\cos \phi, \frac{\mathrm{d} f^{L}}{\mathrm{~d} \phi}\right\rangle_{\phi}(\eta)+\bar{S}(\eta)=F\left\langle\sin \phi, f^{L}\right\rangle_{\phi}(\eta) .
$$

thanks to $\bar{S}=0$. The specular reflexive boundary $f^{L}(L, \phi)=f^{L}(L, R \phi)$ implies $\left\langle\sin \phi, f^{L}\right\rangle_{\phi}(L)=0$. Then we have

$$
\left\langle\sin \phi, f^{L}\right\rangle_{\phi}(\eta)=0
$$

It is easy to see

$$
\left\langle\sin \phi, q^{L}\right\rangle_{\phi}(\eta)=0
$$

Hence, we may derive

$$
\left\langle\sin \phi, r^{L}\right\rangle_{\phi}(\eta)=0
$$

This leads (3.31) and completes the proof of (3.2) 
3.1.2. Infinite Slab with $\bar{S}=0$. We turn to the $\epsilon$-Milne problem in the infinite slab, that is, we will consider the following problem

$$
\left\{\begin{aligned}
\sin \phi \frac{\partial f}{\partial \eta}-F(\eta) \cos \phi \frac{\partial f}{\partial \phi}+f-\bar{f} & =S(\eta, \phi), & & (\eta, \phi) \in[0, \infty) \times[-\pi, \pi) \\
f(0, \phi) & =h(\phi) & & \text { for } \sin \phi>0, \\
\lim _{\eta \rightarrow \infty} f(\eta, \phi) & =f_{\infty}, & &
\end{aligned}\right.
$$

where $h$ and $S$ satisfy the assumption (3.1).

For simplicity, we denote the kinetic part $r$ and the fluid part $q$ for $f$ as well as $r^{L}$ and $q^{L}$ for $f^{L}$.

Lemma 3.3. Assume $\bar{S}(\eta)=0$ for any $\eta \in[0, \infty)$ with (3.11) and (3.12). Then there exists a solution $f(\eta, \phi)$ of the infinite slab problem (3.81), satisfying

$$
\begin{aligned}
\|r\|_{L^{2} L^{2}} & \leq C\left(M+\frac{M}{K}\right)<\infty \\
\langle\sin \phi, r\rangle_{\phi}(\eta) & =0 \text { for any } \eta \in[0, \infty), \\
|q(\eta)| & \leq C\left(1+M+\frac{M}{K}+\|r(\eta, \cdot)\|_{L^{2}}\right) .
\end{aligned}
$$

Also, there exists a constant $q_{\infty}=f_{\infty} \in \mathbb{R}$ such that the following estimates hold,

$$
\begin{aligned}
& \left|q_{\infty}\right| \leq C\left(1+M+\frac{M}{K}\right)<\infty \\
& \left|q(\eta)-q_{\infty}\right| \leq C\left(\|r(\eta, \cdot)\|_{L^{2}}+\int_{\eta}^{\infty}|F(y)|\|r(y, \cdot)\|_{L^{2}} \mathrm{~d} y+\int_{\eta}^{\infty}\|S(y, \cdot)\|_{L^{\infty}} \mathrm{d} y\right) \\
& \int_{0}^{\infty}\left|q(\eta)-q_{\infty}\right|^{2} \mathrm{~d} \eta \leq C\left(M+\frac{M}{K}\right)^{2}<\infty .
\end{aligned}
$$

The solution is unique among functions such that (3.82), (3.85) and (3.87) hold.

Proof. The existence of the solution is obtained by $L \rightarrow \infty$, the estimates (3.82)-(3.87) follow from the equation (3.81) immediately.

Step 1: Existence of the solution and estimates (3.82), (3.83) and (3.84)

By the estimates from Lemma 3.3 , the solution $f^{L}$ of the $(3.26)$ is bounded in $L_{l o c}^{2}\left([0, \infty) ; L^{2}[-\pi, \pi)\right)$. Then there exists a subsequence, which is also denoted as $f^{L}$, such that

$$
q^{L} \rightarrow q, r^{L} \rightarrow r, \text { weakly in } L_{l o c}^{2}\left([0, \infty) ; L^{2}[-\pi, \pi)\right) .
$$

The limit function $f=q+r$ satisfies the equation and the boundary condition at $\eta=0$ in the weak sense. This shows the existence of the solution.

Then property (3.82) naturally holds due to the weak lower semi-continuity of norm $\|\cdot\|_{L^{2} L^{2}}$. The orthogonal relation (3.83) is also preserved.

For the estimate of (3.84), we need the facts that $F \in L^{1}[0, \infty) \cap L^{2}[0, \infty), r \in L^{2}([0, \infty) \times[-\pi, \pi))$ and $S$ exponentially decays at the far field, corresponding to (3.73), (3.82) and (3.12). We use the notation in Step 5 of the proof of Lemma 3.2. Recall (3.63) to (3.32) with $\lambda=0$ and $L=\infty$, we have

$$
\frac{\mathrm{d} \beta}{\mathrm{d} \eta}=D(\eta, \phi),
$$

where

$$
\beta(\eta)=\left\langle\sin ^{2} \phi, f\right\rangle_{\phi}(\eta)
$$

and

$$
D(\eta, \phi)=-\langle\sin \phi, r\rangle_{\phi}-F(\eta)\langle\cos (2 \phi), r\rangle_{\phi}+\langle\sin \phi, S\rangle_{\phi}(\eta) .
$$

The orthogonal relation (3.83) implies

$$
D(\eta, \phi)=-F(\eta)\langle\cos (2 \phi), r\rangle_{\phi}+\langle\sin \phi, S\rangle_{\phi}(\eta) .
$$


Hence, we can integrate (3.89) over $[0, \eta]$ to show

$$
\beta(\eta)-\beta(0)=-\int_{0}^{\eta} F(y)\langle\cos (2 \phi), r\rangle_{\phi}(y) \mathrm{d} y+\int_{0}^{\eta}\langle\sin \phi, S\rangle_{\phi}(y) \mathrm{d} y .
$$

Similar to (3.73), one has

$$
|\beta(0)| \leq 14\left(\|h\|_{L^{2}}+\|S\|_{L^{2} L^{2}}\right)
$$

So, it derives to

$$
\begin{aligned}
|\beta(\eta)| \leq & |\beta(0)|+\int_{0}^{\eta}\left|F(y)\langle\cos (2 \phi), r\rangle_{\phi}(y)\right| \mathrm{d} y+\int_{0}^{\eta}\left|\langle\sin \phi, S\rangle_{\phi}(y)\right| \mathrm{d} y \\
\leq & 14\left(\|h\|_{L^{2}}+\|S\|_{L^{2} L^{2}}\right)+\left(\int_{0}^{\eta}|F(y)|^{2} \mathrm{~d} y\right)^{1 / 2}\left(\int_{0}^{\eta}\|r(y, \cdot)\|_{L^{2}}^{2} \mathrm{~d} y\right)^{1 / 2} \\
& \quad+\pi\left(\int_{0}^{\eta}\|S(y, \cdot)\|_{L^{2}} \mathrm{~d} y\right)^{1 / 2} \cdot \\
\leq & C\left(1+M+\frac{M}{K}\right) .
\end{aligned}
$$

Note that

$$
\beta(\eta)=\left\langle\sin ^{2} \phi, f\right\rangle_{\phi}(\eta)=\left\langle\sin ^{2} \phi, q\right\rangle_{\phi}(\eta)+\left\langle\sin ^{2} \phi, r\right\rangle_{\phi}(\eta)=q(\eta)\|\sin \phi\|_{L^{2}}^{2}+\left\langle\sin ^{2} \phi, r\right\rangle_{\phi}(\eta) .
$$

The inequality (3.84) is valid from (3.82) and the fact (3.12).

Step 2: Estimates (3.85), (3.86) and (3.87) From (3.93) together with the properties of $F$ and $S$, the limit of $\beta(\eta)$ exists. Set $\beta_{\infty}=\lim _{\eta \rightarrow \infty} \beta(\eta)$, from (3.95), we know that

$$
\left|\beta_{\infty}\right| \leq C\left(1+M+\frac{M}{K}\right)
$$

Define the constant as $q_{\infty}=\beta_{\infty} /\|\sin \phi\|_{L^{2}}^{2}=\beta_{\infty} / \pi$, then (3.85) follows directly from (3.97). Moreover,

$$
\beta_{\infty}-\beta(\eta)=\int_{\eta}^{\infty} D(y) \mathrm{d} y=\int_{\eta}^{\infty} F(y)\langle\cos (2 \phi), r\rangle_{\phi}(y) \mathrm{d} y+\int_{\eta}^{\infty}\langle\sin \phi, S\rangle_{\phi}(y) \mathrm{d} y .
$$

Thus, (3.96) yields

$$
\begin{aligned}
& \pi\left|q(\eta)-q_{\infty}\right|=\left|\beta(\eta)-\beta_{\infty}-\left\langle\sin ^{2} \phi, r\right\rangle_{\phi}(\eta)\right| \\
& \quad \leq\left|\left\langle\sin ^{2} \phi, r\right\rangle_{\phi}(\eta)\right|+\int_{\eta}^{\infty}\left|F(y)\langle\cos (2 \phi), r\rangle_{\phi}(y)\right| \mathrm{d} y+\int_{\eta}^{\infty}\left|\langle\sin \phi, S\rangle_{\phi}(y)\right| \mathrm{d} y \\
& \quad \leq \pi\|r(\eta, \cdot)\|_{L^{2}}+\sqrt{\pi} \int_{\eta}^{\infty}|F(y)|\|r(y, \cdot)\|_{L^{2}} \mathrm{~d} y+\sqrt{\pi} \int_{\eta}^{\infty}\|S(y, \cdot)\|_{L^{2}} \mathrm{~d} y .
\end{aligned}
$$

This implies (3.86). Furthermore, we integrate (3.99) over $\eta \in[0, \infty)$. The Cauchy's inequality implies

$$
\int_{0}^{\infty}\left(\int_{\eta}^{\infty}|F(y)|\|r(y, \cdot)\|_{L^{2}} \mathrm{~d} y\right)^{2} \mathrm{~d} \eta \leq\|r\|_{L^{2} L^{2}}^{2} \int_{0}^{\infty} \int_{\eta}^{\infty}|F(y)|^{2} \mathrm{~d} y \mathrm{~d} \eta \leq C .
$$

The exponential decay of $S$ shows that

$$
\int_{0}^{\infty}\left(\int_{\eta}^{\infty}\|S(y, \cdot)\|_{L^{2}} \mathrm{~d} y\right)^{2} \mathrm{~d} \eta \leq C
$$

Hence, the estimate (3.87) naturally follows.

Step 3: Uniqueness 
In order to show the uniqueness of the solution, we assume there are two solutions $f_{1}$ and $f_{2}$ to the equation (3.81) satisfying (3.82) and (3.83). Then $f^{\prime}=f_{1}-f_{2}$ satisfies the equation

$$
\left\{\begin{aligned}
\sin \phi \frac{\partial f^{\prime}}{\partial \eta}-F(\eta) \cos \phi \frac{\partial f^{\prime}}{\partial \phi}+f^{\prime}-\bar{f}^{\prime} & =0 \\
f^{\prime}(0, \phi) & =0 \text { for } \sin \phi>0, \\
\lim _{\eta \rightarrow \infty} f^{\prime}(\eta, \phi) & =f_{\infty}^{\prime} .
\end{aligned}\right.
$$

Similarly, we can define $r^{\prime}$ and $q^{\prime}$. Multiplying $\mathrm{e}^{V(\eta)} f^{\prime}$ on both sides of (3.102) and integrating over $\phi \in$ $[-\pi, \pi)$ yields

$$
\begin{aligned}
& \frac{1}{2} \frac{\mathrm{d}}{\mathrm{d} \eta}\left(\left\langle f^{\prime}, f^{\prime} \sin \phi\right\rangle_{\phi}(\eta) \mathrm{e}^{V(\eta)}\right) \\
= & \left(\left\langle f^{\prime}, \frac{\mathrm{d} f^{\prime}}{\mathrm{d} \eta} \sin \phi\right\rangle_{\phi}(\eta) \mathrm{e}^{V(\eta)}\right)-\frac{1}{2}\left(F(\eta)\left\langle f^{\prime}, f^{\prime} \sin \phi\right\rangle_{\phi}(\eta) \mathrm{e}^{V(\eta)}\right) \\
= & \left(\left\langle f^{\prime}, \frac{\mathrm{d} f^{\prime}}{\mathrm{d} \eta} \sin \phi\right\rangle_{\phi}(\eta) \mathrm{e}^{V(\eta)}\right)-\left(F(\eta)\left\langle f^{\prime}, \frac{\mathrm{d} f^{\prime}}{\mathrm{d} \phi} \cos \phi\right\rangle_{\phi}(\eta) \mathrm{e}^{V(\eta)}\right) \\
= & \left\langle f^{\prime}-\bar{f}^{\prime}, f^{\prime}\right\rangle_{\phi}(\eta) \mathrm{e}^{V(\eta)}=-\left(\left\|r^{\prime}(\eta, \cdot)\right\|_{L^{2}}^{2} \mathrm{e}^{V(\eta)}\right) \leq 0 . .
\end{aligned}
$$

This implies that $\gamma(\eta)=\frac{1}{2}\left\langle f^{\prime}, f^{\prime} \sin \phi\right\rangle_{\phi}(\eta) \mathrm{e}^{V(\eta)}$ is decreasing. Since $r^{\prime} \in L^{2}([0, \infty) \times[-\pi, \pi))$ and $q^{\prime}-q_{\infty}^{\prime} \in$ $L^{2}([0, \infty) \times[-\pi, \pi))$, there exists a subsequence $\eta_{k}$ such that $\left\|r^{\prime}\left(\eta_{k}, \cdot\right)\right\|_{L^{2}} \rightarrow 0$ and $q^{\prime}\left(\eta_{k}\right)-q_{\infty}^{\prime} \rightarrow 0$ as $k \rightarrow \infty$. Hence, this implies

$$
\frac{1}{2}\left\langle r^{\prime}, r^{\prime} \sin \phi\right\rangle_{\phi}\left(\eta_{k}\right) \mathrm{e}^{V\left(\eta_{k}\right)} \rightarrow 0, \quad k \rightarrow \infty .
$$

Also, due to the fact that $q^{\prime}\left(\eta_{k}\right)$ is independent of $\phi$ and it is finite dimension with respect to $\phi$, we have

$$
\gamma\left(\eta_{k}\right) \rightarrow 0, \quad k \rightarrow \infty
$$

By the monotonicity, $\gamma(\eta)$ decreases to zero and $\gamma(\eta) \geq 0$. Then we can integrate (3.103) over $\eta \in[0, \infty)$ to obtain

$$
\gamma(\infty)-\gamma(0)=-2 \int_{0}^{\infty}\left\|r^{\prime}(y, \cdot)\right\|_{L^{2}}^{2} \mathrm{e}^{V(y)} \mathrm{d} y
$$

which implies

$$
\gamma(0)=\left\langle f^{\prime}, f^{\prime} \sin \phi\right\rangle_{\phi}(0) \mathrm{e}^{V(0)}=2 \int_{0}^{\infty}\left\|r^{\prime}(y, \cdot)\right\|_{L^{2}}^{2} e^{V(y)} \mathrm{d} y
$$

Also, we know

$$
0 \leq \frac{1}{2}\left\langle f^{\prime}, f^{\prime} \sin \phi\right\rangle_{\phi}(0) \mathrm{e}^{-V(0)}=\frac{1}{2}\left\langle f^{\prime}, f^{\prime} \sin \phi\right\rangle_{\phi}(0) \leq \int_{\sin \phi>0}\left(f^{\prime}(0, \phi)\right)^{2} \sin \phi \mathrm{d} \phi=0 .
$$

Naturally, we have

$$
\left\langle f^{\prime}, f^{\prime} \sin \phi\right\rangle_{\phi}(0) \mathrm{e}^{V(0)}=2 \int_{0}^{\infty}\left\|r^{\prime}(y, \cdot)\right\|_{L^{2}}^{2} \mathrm{e}^{V(y)} \mathrm{d} y=0 .
$$

Hence, we have $r^{\prime}=0$ and $f^{\prime}(0, \phi)=0$. Thus, $f^{\prime}(\eta, \phi)=q^{\prime}(\eta)$. Plugging this into the equation (3.102) reveals $\partial_{\eta} q^{\prime}=0$. Therefore, $f^{\prime}(\eta, \phi)=C$ for all $(\eta, \phi) \in[0, \infty) \times[-\pi, \pi]$. Naturally the boundary data leads to $C=0$, which derives to $f^{\prime}=0$. That is, $f_{1}=f_{2}$ and the uniqueness of the solution to (3.102) follows directly.

3.1.3. $\bar{S} \neq 0$ Case. Consider the $\epsilon$-Milne problem for $f(\eta, \phi)$ in $(\eta, \phi) \in[0, \infty) \times[-\pi, \pi)$ with a general source term

$$
\left\{\begin{aligned}
\sin \phi \frac{\partial f}{\partial \eta}-F(\eta) \cos \phi \frac{\partial f}{\partial \phi}+f-\bar{f} & =S(\eta, \phi), \\
f(0, \phi) & =h(\phi) \text { for } \sin \phi>0, \\
\lim _{\eta \rightarrow \infty} f(\eta, \phi) & =f_{\infty} .
\end{aligned}\right.
$$


Lemma 3.4. Assume (3.11) and (3.12) hold. Then there exists a unique solution $f(\eta, \phi)$ of the problem (3.110), satisfying

$$
\begin{aligned}
\|r\|_{L^{2} L^{2}} & <C\left(1+M+\frac{M}{K}\right) \leq \infty, \\
\langle\sin \phi, r\rangle_{\phi}(\eta) & =-\int_{\eta}^{\infty} \mathrm{e}^{V(y)-V(\eta)} \bar{S}(y) \mathrm{d} y, \\
|q(\eta)| & \leq C\left(1+M+\frac{M}{K}+\|r(\eta, \cdot)\|_{L^{2}}\right) .
\end{aligned}
$$

Also there exists a constant $q_{\infty}=f_{\infty} \in \mathbb{R}$ such that the following estimates hold,

$$
\begin{aligned}
\left|q_{\infty}\right| & \leq C\left(1+M+\frac{M}{K}\right)<\infty \\
\left|q(\eta)-q_{\infty}\right| & \leq C\left(\|r(\eta, \cdot)\|_{L^{2}}+\int_{\eta}^{\infty}|F(y)|\|r(y, \cdot)\|_{L^{2}} \mathrm{~d} y+\int_{\eta}^{\infty}\|S(y, \cdot)\|_{L^{2}} \mathrm{~d} y\right), \\
\left\|q(\cdot)-q_{\infty}\right\|_{L^{2}} & \leq C\left(1+M+\frac{M}{K}\right)<\infty .
\end{aligned}
$$

The solution is unique among functions satisfying $\left\|f-f_{\infty}\right\|_{L^{2} L^{2}}<\infty$.

Proof. We can apply superposition property for this linear problem, i.e. write $S=\bar{S}+(S-\bar{S})=S_{Q}+S_{R}$. Then we solve the problem by the following steps.

Step 1: Construction of auxiliary function $f^{1}$

For the zero mean part $S_{R}$, we choose $f^{1}$ as the solution to

$$
\left\{\begin{aligned}
\sin \phi \frac{\partial f^{1}}{\partial \eta}-F(\eta) \cos \phi \frac{\partial f^{1}}{\partial \phi}+f^{1}-\bar{f}^{1} & =S_{R}(\eta, \phi) \\
f^{1}(0, \phi) & =h(\phi) \text { for } \sin \phi>0 \\
\lim _{\eta \rightarrow \infty} f^{1}(\eta, \phi) & =f_{\infty}^{1} .
\end{aligned}\right.
$$

Since $\bar{S}_{R}=0$, by Lemma 3.3, we know there exists a unique solution $f^{1}$ satisfying the $L^{2}$ estimates (3.111), (3.114), (3.115) and (3.116).

Step 2: Construction of auxiliary function $f^{2}$

For the part $S_{Q}$, We seek a function $f^{2}$ satisfying

$$
-\frac{1}{2 \pi} \int_{-\pi}^{\pi}\left(\sin \phi \frac{\partial f^{2}}{\partial \eta}-F(\eta) \cos \phi \frac{\partial f^{2}}{\partial \phi}\right) \mathrm{d} \phi+S_{Q}=0 .
$$

An integration by parts transforms the equation (3.118) into

$$
-\int_{-\pi}^{\pi} \sin \phi \frac{\partial f^{2}}{\partial \eta} \mathrm{d} \phi+\int_{-\pi}^{\pi} F(\eta) \sin \phi f^{2} \mathrm{~d} \phi+2 \pi S_{Q}=0 .
$$

By Setting

$$
f^{2}(\phi, \eta)=a(\eta) \sin \phi
$$

and plugging this ansatz into (3.119), we have

$$
-\frac{\mathrm{d} a}{\mathrm{~d} \eta} \int_{-\pi}^{\pi} \sin ^{2} \phi \mathrm{d} \phi+F(\eta) a(\eta) \int_{-\pi}^{\pi} \sin ^{2} \phi \mathrm{d} \phi+2 \pi S_{Q}=0 .
$$

Hence, we have

$$
-\frac{\mathrm{d} a}{\mathrm{~d} \eta}+F(\eta) a(\eta)+2 S_{Q}=0 .
$$

By assume $a(\infty)=0$, we can directly solve it to obtain

$$
a(\eta)=-\mathrm{e}^{\int_{0}^{\eta} F(y) \mathrm{d} y} \int_{\eta}^{\infty} \mathrm{e}^{-\int_{0}^{y} F(z) \mathrm{d} z} 2 S_{Q}(y) \mathrm{d} y .
$$


In particular, for $\eta=0$, we have

$$
a(0)=-\int_{0}^{\infty} \mathrm{e}^{-\int_{0}^{y} F(z) \mathrm{d} z} 2 S_{Q}(y) \mathrm{d} y .
$$

Based on the exponential decay of $S_{Q}$, we can directly verify $a(\eta)$ decays exponentially to zero as $\eta \rightarrow \infty$ and $f^{2}$ satisfies the estimates (3.111), (3.114), (3.115) and (3.116).

Step 3: Construction of auxiliary function $f^{3}$

Since the boundary condition has been changed, we construct $f^{3}$ verify

$$
\left\{\begin{aligned}
\sin \phi \frac{\partial f^{3}}{\partial \eta}-F(\eta) \cos \phi \frac{\partial f^{3}}{\partial \phi}+f^{3}-\bar{f}^{3} & =-\sin \phi \frac{\partial f^{2}}{\partial \eta}+F(\eta) \cos \phi \frac{\partial f^{2}}{\partial \phi}-f^{2}+\bar{f}^{2}+S_{Q}, \\
f^{3}(0, \phi) & =-a(0) \sin \phi \text { for } \sin \phi>0, \\
\lim _{\eta \rightarrow \infty} f^{3}(\eta, \phi) & =f_{\infty}^{3} .
\end{aligned}\right.
$$

Since the source term satisfy

$$
\int_{-\pi}^{\pi}\left(-\sin \phi \frac{\partial f^{2}}{\partial \eta}+F(\eta) \cos \phi \frac{\partial f^{2}}{\partial \phi}-f^{2}+\bar{f}^{2}+S_{Q}\right) \mathrm{d} \phi=0
$$

we can apply Lemma 3.3 to obtain a unique solution $f^{3}$ satisfying the estimates (3.111), (3.114), (3.115) and (3.116).

Step 4: Construction of auxiliary function $f^{4}$

We now define $f^{4}=f^{2}+f^{3}$ and an explicit verification shows

$$
\left\{\begin{aligned}
\sin \phi \frac{\partial f^{4}}{\partial \eta}-F(\eta) \cos \phi \frac{\partial f^{4}}{\partial \phi}+f^{4}-\bar{f}^{4} & =S_{Q}(\eta, \phi) \\
f^{4}(0, \phi) & =0 \text { for } \sin \phi>0 \\
\lim _{\eta \rightarrow \infty} f^{4}(\eta, \phi) & =f_{\infty}^{4}
\end{aligned}\right.
$$

and $f^{4}$ satisfies the $L^{2}$ estimates (3.111), (3.114), (3.115) and (3.116).

In summary, we deduce that $f^{1}+f^{4}$ is the solution of (3.110) and satisfies the estimates (3.111), (3.114), (3.115) and (3.116). A direct computation of $\left\langle\sin \phi, f^{i}\right\rangle_{\phi}(\eta)$ for $i=1,2,3,4$ leads to (3.112). From $\left\|f-f_{\infty}\right\|_{L^{2} L^{2}}<\infty$, we deduce $\left\|\bar{f}(\cdot)-f_{\infty}\right\|_{L^{2}}<\infty$. Set $f_{\infty}=f_{\infty}^{1}+f_{\infty}^{4}$, a similar argument in Lemma 3.3 shows that the uniqueness of solution.

Combining all above, we have the following theorem.

Theorem 3.5. Assume (3.11) and (3.12) hold, there exists a unique solution $f(\eta, \phi)$ for the $\epsilon$-Milne problem (3.8), which satisfies the estimates

$$
\left\|f-f_{\infty}\right\|_{L^{2} L^{2}} \leq C\left(1+M+\frac{M}{K}\right)<\infty,
$$

for some real number $f_{\infty}$ such that

$$
\left|f_{\infty}\right| \leq C\left(1+M+\frac{M}{K}\right)<\infty .
$$

3.2. $L^{\infty}$ Estimates. For the analysis of the $\epsilon$-Milne problem, we need the estimate of $\|f\|_{L^{\infty} L^{\infty}}$. So we will consider the $L^{\infty}$ estimates in the following subsection.

3.2.1. Finite Slab. We firstly consider the penalty $\epsilon$-transport problem in a finite slab $(\eta, \phi) \in[0, L] \times[-\pi, \pi)$

$$
\left\{\begin{aligned}
\lambda f_{\lambda}^{L}+\sin \phi \frac{\partial f_{\lambda}^{L}}{\partial \eta}-F(\eta) \cos \phi \frac{\partial f_{\lambda}^{L}}{\partial \phi}+f_{\lambda}^{L} & =H(\eta, \phi), \\
f_{\lambda}^{L}(0, \phi) & =h(\phi), \quad \text { for } \sin \phi>0, \\
f_{\lambda}^{L}(L, R \phi) & =f_{\lambda}^{L}(L, \phi)
\end{aligned}\right.
$$

with $R \phi=-\phi$. We have the following result. 
Lemma 3.6. Assume $\|H\|_{L^{\infty} L^{\infty}}<\infty$ and $\|h\|_{L^{\infty}}<\infty$, then there exists a solution $f_{\lambda}^{L}(\eta, \phi)$ to the penalized $\epsilon$-transport equation (3.130) satisfying

$$
\left\|f_{\lambda}^{L}\right\|_{L^{\infty} L^{\infty}} \leq\|h\|_{L^{\infty}}+\|H\|_{L^{\infty} L^{\infty}} .
$$

Proof. Define the energy as follows:

$$
E(\eta, \phi)=\cos \phi e^{V(\eta)}
$$

In the plane $(\eta, \phi) \in[0, \infty) \times[-\pi, \pi)$, on the curve $\phi=\phi(\eta)$ with constant energy, we can see

$$
\frac{\mathrm{d} E}{\mathrm{~d} \eta}=\frac{\partial E}{\partial \eta}+\frac{\partial E}{\partial \phi} \frac{\partial \phi}{\partial \eta}=-\cos \phi F(\eta) \mathrm{e}^{V(\eta)}-\sin \phi \mathrm{e}^{V(\eta)} \frac{\partial \phi}{\partial \eta}=0
$$

which further implies

$$
\frac{\partial \phi}{\partial \eta}=-\frac{\cos \phi F(\eta)}{\sin \phi}
$$

Plugging this into the equation (3.130), on this curve, we deduce

$$
\frac{\mathrm{d} f}{\mathrm{~d} \eta}=\frac{\partial f}{\partial \eta}+\frac{\partial f}{\partial \phi} \frac{\partial \phi}{\partial \eta}=\frac{1}{\sin \phi}\left(\sin \phi \frac{\partial f}{\partial \eta}-\cos \phi F(\eta) \frac{\partial f}{\partial \phi}\right) .
$$

Hence, this curve with constant energy is exactly the characteristics of the equation (3.130). Along this curve, the equation can be simplified as follows:

$$
\lambda f_{\lambda}+\sin \phi \frac{\mathrm{d} f_{\lambda}}{\mathrm{d} \eta}+f_{\lambda}=H .
$$

An implicit function $\eta^{+}(\eta, \phi)$ can be determined through

$$
|E(\eta, \phi)|=\mathrm{e}^{V\left(\eta^{+}\right)} .
$$

which means $\left(\eta^{+}, \phi_{0}\right)$ with $\sin \phi_{0}=0$ is on the same characteristics as $(\eta, \phi)$. We also define the quantities for $0 \leq \eta^{+} \leq \eta^{\prime} \leq \eta$ as follows:

$$
\begin{aligned}
\phi^{\prime}\left(\phi, \eta, \eta^{\prime}\right) & =\cos ^{-1}\left(\cos \phi \mathrm{e}^{V(\eta)-V\left(\eta^{\prime}\right)}\right), \\
R \phi^{\prime}\left(\phi, \eta, \eta^{\prime}\right) & =-\cos ^{-1}\left(\cos \phi \mathrm{e}^{V(\eta)-V\left(\eta^{\prime}\right)}\right)=-\phi^{\prime}\left(\phi, \eta, \eta^{\prime}\right),
\end{aligned}
$$

where the inverse trigonometric function can be defined single-valued in the domain $[0, \pi)$ and the quantities are always well-defined due to the monotonicity of $V$. Finally, we denote

$$
G_{\eta, \eta^{\prime}}^{\lambda}(\phi)=\int_{\eta^{\prime}}^{\eta} \frac{1+\lambda}{\sin \left(\phi^{\prime}(\phi, \eta, \xi)\right)} \mathrm{d} \xi
$$

With these notations, we can define the solution to (3.130) along the characteristics as follows:

Case I. For $\sin \phi>0$ and $|E(\eta, \phi)| \leq 1$,

$$
f_{\lambda}^{L}(\eta, \phi)=h\left(\phi^{\prime}(\phi, \eta, 0)\right) \exp \left(-G_{\eta, 0}^{\lambda}\right)+\int_{0}^{\eta} \frac{H\left(\eta^{\prime}, \phi^{\prime}\left(\phi, \eta, \eta^{\prime}\right)\right)}{\sin \left(\phi^{\prime}\left(\phi, \eta, \eta^{\prime}\right)\right)} \exp \left(-G_{\eta, \eta^{\prime}}^{\lambda}\right) \mathrm{d} \eta^{\prime} .
$$

Case II. For $\sin \phi>0$ and $|E(\eta, \phi)| \geq 1$,

$$
f_{\lambda}^{L}(\eta, \phi)=f_{\lambda}^{L}\left(\eta^{+}(\eta, \phi), \phi_{0}\right) \exp \left(-G_{\eta, \eta^{+}}^{\lambda}\right)+\int_{\eta^{+}}^{\eta} \frac{H\left(\eta^{\prime}, \phi^{\prime}\left(\phi, \eta, \eta^{\prime}\right)\right)}{\sin \left(\phi^{\prime}\left(\phi, \eta, \eta^{\prime}\right)\right)} \exp \left(-G_{\eta, \eta^{\prime}}^{\lambda}\right) \mathrm{d} \eta^{\prime} .
$$

Case III. For $\sin \phi<0$ and $|E(\eta, \phi)| \geq 1$,

$$
f_{\lambda}^{L}(\eta, \phi)=f_{\lambda}^{L}\left(\eta^{+}(\eta, \phi), \phi_{0}\right) \exp \left(-G_{\eta, \eta^{+}}^{\lambda}\right)+\int_{\eta^{+}}^{\eta} \frac{H\left(\eta^{\prime}, \phi^{\prime}\left(\phi, \eta, \eta^{\prime}\right)\right)}{\sin \left(\phi^{\prime}\left(\phi, \eta, \eta^{\prime}\right)\right)} \exp \left(-G_{\eta, \eta^{\prime}}^{\lambda}\right) \mathrm{d} \eta^{\prime} .
$$


Case IV. For $\sin \phi<0$ and $|E(\eta, \phi)| \leq 1$,

$$
\begin{aligned}
f_{\lambda}^{L}(\eta, \phi)= & h\left(\phi^{\prime}(\phi, \eta, 0)\right) \exp \left[-G_{L, 0}^{\lambda}-G_{L, \eta}^{\lambda}\right] \\
& +\int_{0}^{L} \frac{H\left(\eta^{\prime}, R \phi^{\prime}\left(\phi, \eta, \eta^{\prime}\right)\right)}{\sin \left(\phi^{\prime}\left(\phi, \eta, \eta^{\prime}\right)\right)} \exp \left[-G_{L, \eta^{\prime}}^{\lambda}-G_{L, \eta}^{\lambda}\right] \mathrm{d} \eta^{\prime} \\
& +\int_{\eta}^{L} \frac{H\left(\eta^{\prime}, \phi^{\prime}\left(\phi, \eta, \eta^{\prime}\right)\right)}{\sin \left(\phi^{\prime}\left(\phi, \eta, \eta^{\prime}\right)\right)} \exp \left(G_{\eta, \eta^{\prime}}^{\lambda}\right) \mathrm{d} \eta^{\prime} .
\end{aligned}
$$

In the following, we give the estimate of (3.131). In Case I, (3.141) derives to

$$
\begin{aligned}
\left\|f_{\lambda}^{L}\right\|_{L^{\infty} L^{\infty}} & \leq\|h\|_{L^{\infty}} \exp \left(-G_{\eta, 0}^{\lambda}\right)+\|H\|_{L^{\infty} L^{\infty}} \int_{0}^{\eta} \frac{\exp \left(-G_{\eta, \eta^{\prime}}^{\lambda}\right)}{\sin \left(\phi^{\prime}\left(\phi, \eta, \eta^{\prime}\right)\right)} \mathrm{d} \eta^{\prime} \\
& =\|h\|_{L^{\infty}} \exp \left(-G_{\eta, 0}^{\lambda}\right)+\|H\|_{L^{\infty} L^{\infty}} \frac{1}{1+\lambda}\left(1-\exp \left(-G_{\eta, 0}^{\lambda}\right)\right) \\
& \leq\|h\|_{L^{\infty}}+\|H\|_{L^{\infty} L^{\infty}} .
\end{aligned}
$$

In Case II and III, the main difficulty is the lack of estimate for $f_{\lambda}^{L}\left(\eta^{+}(\eta, \phi), \phi_{0}\right)$. But, we denote the points $\left(\eta^{+}, \phi_{0}\right),\left(L, \phi_{L}\right)$, and $\left(L,-\phi_{L}\right)$ with

$$
\phi_{L}=\cos ^{-1}\left(\mathrm{e}^{V\left(\eta^{+}(\eta, \phi)\right)-V(L)}\right)>0
$$

are on the same characteristic line (3.132). Then, along this characteristic line, we can compute that

$$
\begin{aligned}
& f_{\lambda}^{L}\left(\eta^{+}, \phi_{0}\right)=f_{\lambda}^{L}\left(L, \phi_{L}\right) \exp \left(G_{L, \eta^{+}}^{\lambda}\left(\phi_{L}\right)\right)-\int_{\eta^{+}}^{L} \frac{H\left(\eta^{\prime}, \phi^{\prime}\left(\phi_{L}, \eta, \eta^{\prime}\right)\right)}{\sin \left(\phi^{\prime}\left(\phi_{L}, \eta, \eta^{\prime}\right)\right)} \exp \left(G_{\eta^{\prime}, \eta^{+}}^{\lambda}\left(\phi_{L}\right)\right) \mathrm{d} \eta^{\prime}, \\
& f_{\lambda}^{L}\left(\eta^{+}, \phi_{0}\right)=f_{\lambda}^{L}\left(L,-\phi_{L}\right) \exp \left(R G_{L, \eta^{+}}^{\lambda}\left(\phi_{L}\right)\right)-\int_{\eta^{+}}^{L} \frac{H\left(\eta^{\prime}, R \phi^{\prime}\left(\phi_{L}, \eta, \eta^{\prime}\right)\right)}{\sin \left(\phi^{\prime}\left(\phi_{L}, \eta, \eta^{\prime}\right)\right)} \exp \left(R G_{\eta^{\prime}, \eta^{+}}^{\lambda}\left(\phi_{L}\right)\right) \mathrm{d} \eta^{\prime} .
\end{aligned}
$$

Then naturally we have

$$
\begin{aligned}
& f_{\lambda}^{L}\left(L, \phi_{L}\right) \exp \left(G_{L, \eta^{+}}^{\lambda}\left(\phi_{L}\right)\right)-\int_{\eta^{+}}^{L} \frac{H\left(\eta^{\prime}, \phi^{\prime}\left(\phi_{L}, L, \eta^{\prime}\right)\right)}{\sin \left(\phi^{\prime}\left(\phi_{L}, L, \eta^{\prime}\right)\right)} \exp \left(G_{\eta^{\prime}, \eta^{+}}^{\lambda}\left(\phi_{L}\right)\right) \mathrm{d} \eta^{\prime} \\
= & f_{\lambda}^{L}\left(L,-\phi_{L}\right) \exp \left(-G_{L, \eta^{+}}^{\lambda}\left(\phi_{L}\right)\right)-\int_{\eta^{+}}^{L} \frac{H\left(\eta^{\prime}, R \phi^{\prime}\left(\phi_{L}, L, \eta^{\prime}\right)\right)}{\sin \left(\phi^{\prime}\left(\phi_{L}, L, \eta^{\prime}\right)\right)} \exp \left(-G_{\eta^{\prime}, \eta^{+}}^{\lambda}\left(\phi_{L}\right)\right) \mathrm{d} \eta^{\prime} .
\end{aligned}
$$

The specular reflective boundary condition implies $f_{\lambda}^{L}\left(L, \phi_{L}\right)=f_{\lambda}^{L}\left(L,-\phi_{L}\right)$. Then we obtain

$$
\begin{aligned}
f_{\lambda}^{L}\left(L, \phi_{L}\right)= & \frac{1}{\exp \left(G_{L, \eta^{+}}^{\lambda}\left(\phi_{L}\right)\right)-\exp \left(-G_{L, \eta^{+}}^{\lambda}\left(\phi_{L}\right)\right)}\left(\int_{\eta^{+}}^{L} \frac{H\left(\eta^{\prime}, \phi^{\prime}\left(\phi_{L}, L, \eta^{\prime}\right)\right)}{\sin \left(\phi^{\prime}\left(\phi_{L}, L, \eta^{\prime}\right)\right)} \exp \left(G_{\eta^{\prime}, \eta^{+}}^{\lambda}\left(\phi_{L}\right)\right) \mathrm{d} \eta^{\prime}\right. \\
& \left.-\int_{\eta^{+}}^{L} \frac{H\left(\eta^{\prime}, R \phi^{\prime}\left(\phi_{L}, L, \eta^{\prime}\right)\right)}{\sin \left(\phi^{\prime}\left(\phi_{L}, L, \eta^{\prime}\right)\right)} \exp \left(-G_{\eta^{\prime}, \eta^{+}}^{\lambda}\left(\phi_{L}\right)\right) \mathrm{d} \eta^{\prime}\right) .
\end{aligned}
$$

It naturally leads to

$$
\left|f_{\lambda}^{L}\left(\eta^{+}, \phi_{0}\right)\right| \leq\|H\|_{L^{\infty} L^{\infty}} \frac{\int_{\eta^{+}}^{L} \frac{\exp \left(-G_{L, \eta^{\prime}}^{\lambda}\left(\phi_{L}\right)\right)}{\sin \left(\phi^{\prime}\left(\phi_{L}, L, \eta^{\prime}\right)\right)} \mathrm{d} \eta^{\prime}+\int_{\eta^{+}}^{L} \frac{\exp \left(G_{L, \eta^{\prime}}^{\lambda}\left(\phi_{L}\right)\right)}{\sin \left(\phi^{\prime}\left(\phi_{L}, L, \eta^{\prime}\right)\right)} \mathrm{d} \eta^{\prime}}{\exp \left(G_{L, \eta^{+}}^{\lambda}\left(\phi_{L}\right)\right)-\exp \left(-G_{L, \eta^{+}}^{\lambda}\left(\phi_{L}\right)\right)} \leq\|H\|_{L^{\infty} L^{\infty}} .
$$

Similar to the estimates in Case I, we have

$$
\left\|f_{\lambda}^{L}\right\|_{L^{\infty} L^{\infty}} \leq\left(\exp \left(-G_{L, \eta^{+}}^{\lambda}\left(\phi_{L}\right)\right)+\int_{\eta^{+}}^{L} \frac{\exp \left(-G_{L, \eta^{\prime}}^{\lambda}\left(\phi_{L}\right)\right)}{\sin \left(\phi^{\prime}\left(\phi_{L}, L, \eta^{\prime}\right)\right)} \mathrm{d} \eta^{\prime}\right)\|H\|_{L^{\infty} L^{\infty}} \leq\|H\|_{L^{\infty} L^{\infty}} .
$$


In case IV, it is similar to Case I, we can directly estimate to obtain

$$
\left\|f_{\lambda}^{L}\right\|_{L^{\infty} L^{\infty}} \leq\|h\|_{L^{\infty}}+\|H\|_{L^{\infty} L^{\infty}} .
$$

Summarizing all above, we complete the proof of (3.131).

3.2.2. Infinite Slab. Let $L \rightarrow \infty$, we consider the following problem in the infinite slab $\eta \in(0, \infty)$,

$$
\left\{\begin{array}{rlrl}
\lambda f_{\lambda}+\sin \phi \frac{\partial f_{\lambda}}{\partial \eta}-F(\eta) \cos \phi \frac{\partial f_{\lambda}}{\partial \phi}+f_{\lambda} & =H(\eta, \phi), & \eta>0 \\
f_{\lambda}(0, \phi) & =h(\phi) & & \text { for } \sin \phi>0 \\
\lim _{\eta \rightarrow \infty} f_{\lambda} & =f_{\infty} . & &
\end{array}\right.
$$

The following lemma holds.

Lemma 3.7. $\|H\|_{L^{\infty} L^{\infty}}<\infty$ and $\|h\|_{L^{\infty}}<\infty$, then the solution $f_{\lambda}(\eta, \phi)$ to the penalized $\epsilon$-Milne equation (3.154) satisfies

$$
\left\|f_{\lambda}\right\|_{L^{\infty} L^{\infty}} \leq C\left(\|h\|_{L^{\infty}}+\|H\|_{L^{\infty} L^{\infty}}\right)
$$

where $C$ is a constant independent of $\lambda$.

Now, we use the fact that

$$
\lim _{L \rightarrow \infty} \exp \left(-G_{L, \eta}^{\lambda}\right)=0 \text { for } \sin \phi<0
$$

It can be defined the solution via taking limit $L \rightarrow \infty$ in (3.141)-(3.144) as follows:

$$
f_{\lambda}(\eta, \phi)=\mathcal{A}_{\lambda}[h(\phi)]+\mathcal{T}_{\lambda}[H(\eta, \phi)]
$$

where

Case I. For $\sin \phi>0$ and $|E(\eta, \phi)| \leq 1$,

$$
\begin{aligned}
\mathcal{A}_{\lambda}[h(\phi)] & =h\left(\phi^{\prime}(\phi, \eta, 0)\right) \exp \left(-G_{\eta, 0}^{\lambda}\right) \\
\mathcal{T}_{\lambda}[H(\eta, \phi)] & =\int_{0}^{\eta} \frac{H\left(\eta^{\prime}, \phi^{\prime}\left(\phi, \eta, \eta^{\prime}\right)\right)}{\sin \left(\phi^{\prime}\left(\phi, \eta, \eta^{\prime}\right)\right)} \exp \left(-G_{\eta, \eta^{\prime}}^{\lambda}\right) \mathrm{d} \eta^{\prime} .
\end{aligned}
$$

Case II. For $\sin \phi>0$ and $|E(\eta, \phi)| \geq 1$,

$$
\begin{aligned}
\mathcal{A}_{\lambda}[h(\phi)]= & 0 \\
\mathcal{T}_{\lambda}[H(\eta, \phi)]= & f_{\lambda}\left(\eta^{+}, \phi_{0}\right) \exp \left(-G_{\eta, \eta^{+}}^{\lambda}\right)+\int_{\eta^{+}}^{\eta} \frac{H\left(\eta^{\prime}, \phi^{\prime}\left(\phi, \eta, \eta^{\prime}\right)\right)}{\sin \left(\phi^{\prime}\left(\phi, \eta, \eta^{\prime}\right)\right)} \exp \left(-G_{\eta, \eta^{\prime}}^{\lambda}\right) \mathrm{d} \eta^{\prime}, \\
= & \int_{\eta^{+}}^{\infty} \frac{H\left(\eta^{\prime}, R \phi^{\prime}\left(\phi, \eta, \eta^{\prime}\right)\right)}{\sin \left(\phi^{\prime}\left(\phi, \eta, \eta^{\prime}\right)\right)} \exp \left(G_{\eta, \eta^{\prime}}^{\lambda}-G_{\eta, \eta^{+}}^{\lambda}\right) \mathrm{d} \eta^{\prime} \\
& +\int_{\eta^{+}}^{\eta} \frac{H\left(\eta^{\prime}, \phi^{\prime}\left(\phi, \eta, \eta^{\prime}\right)\right)}{\sin \left(\phi^{\prime}\left(\phi, \eta, \eta^{\prime}\right)\right)} \exp \left(-G_{\eta, \eta^{\prime}}^{\lambda}\right) \mathrm{d} \eta^{\prime} .
\end{aligned}
$$

Case III. For $\sin \phi<0$,

$$
\begin{aligned}
\mathcal{A}_{\lambda}[h(\phi)] & =0 \\
\mathcal{T}_{\lambda}[H(\eta, \phi)] & =\int_{\eta}^{\infty} \frac{H\left(\eta^{\prime}, R \phi^{\prime}\left(\phi, \eta, \eta^{\prime}\right)\right)}{\sin \left(\phi^{\prime}\left(\phi, \eta, \eta^{\prime}\right)\right)} \exp \left(G_{\eta, \eta^{\prime}}^{\lambda}\right) \mathrm{d} \eta^{\prime} .
\end{aligned}
$$

In Case II, we replace $f_{\lambda}\left(\eta^{+}, \phi_{0}\right)$ by the integral along the characteristics in $\sin \phi<0$. Also, the latter two cases are combined into a united one. In order to achieve the estimate of $f_{\lambda}$, we need to give several technical lemmas about $\mathcal{A}_{\lambda}$ and $\mathcal{T}_{\lambda}$. From (3.158)-(3.162), one can easily obtain

Lemma 3.8. The operator $\mathcal{A}_{\lambda}$ satisfies

$$
\left\|\mathcal{A}_{\lambda}[h(\phi)]\right\|_{L^{\infty} L^{\infty}} \leq\|h\|_{L^{\infty}} .
$$


Proof. Since $\sin \left(\phi^{\prime}(\phi, \eta, \xi)\right)>0$ for $0 \leq \xi \leq \eta$, we know that

$$
\exp \left(-G_{\eta, 0}^{\lambda}\right) \leq 1
$$

Then, the estimate of (3.164) holds immediately.

Lemma 3.9. There exists some constant such that the integral operator $\mathcal{T}_{\lambda}$ satisfies

$$
\left\|\mathcal{T}_{\lambda}[H]\right\|_{L^{\infty} L^{\infty}} \leq C\|H\|_{L^{\infty} L^{\infty}} \quad \text { for all } \lambda>0 .
$$

Proof. The proof of this lemma will divided into three cases.

In Case I, it implies that $\sin \phi>0$ and $|E(\eta, \phi)| \leq 1$. One has

$$
\begin{aligned}
\left|\mathcal{T}_{\lambda}[H]\right| & \leq \int_{0}^{\eta}\left|H\left(\eta^{\prime}, \phi^{\prime}\left(\phi, \eta, \eta^{\prime}\right)\right)\right| \frac{1}{\sin \left(\phi^{\prime}\left(\phi, \eta, \eta^{\prime}\right)\right)} \exp \left(-G_{\eta, \eta^{\prime}}^{\lambda}\right) \mathrm{d} \eta^{\prime} \\
& \leq\|H\|_{L^{\infty} L^{\infty}} \int_{0}^{\eta} \frac{1}{\sin \left(\phi^{\prime}\left(\phi, \eta, \eta^{\prime}\right)\right)} \exp \left(-G_{\eta, \eta^{\prime}}^{\lambda}\right) \mathrm{d} \eta^{\prime} .
\end{aligned}
$$

We can directly estimate

$$
\int_{0}^{\eta} \frac{1}{\sin \left(\phi^{\prime}\left(\phi, \eta, \eta^{\prime}\right)\right)} \exp \left(-G_{\eta, \eta^{\prime}}^{\lambda}\right) \mathrm{d} \eta^{\prime} \leq \frac{1}{1+\lambda} \int_{0}^{\infty} \mathrm{e}^{-z} \mathrm{~d} z \leq \frac{1}{1+\lambda},
$$

and (3.165) naturally follows.

In Case III, it implies that $\sin \phi<0$. So it holds that

$$
\begin{aligned}
\left|\mathcal{T}_{\lambda}[H]\right| & \leq \int_{\eta}^{\infty}\left|H\left(\eta^{\prime}, \phi^{\prime}\left(\phi, \eta, \eta^{\prime}\right)\right)\right| \frac{1}{\sin \left(\phi^{\prime}\left(\phi, \eta, \eta^{\prime}\right)\right)} \exp \left(G_{\eta, \eta^{\prime}}^{\lambda}\right) \mathrm{d} \eta^{\prime} \\
& \leq\|H\|_{L^{\infty} L^{\infty}} \int_{\eta}^{\infty} \frac{1}{\sin \left(\phi^{\prime}\left(\phi, \eta, \eta^{\prime}\right)\right)} \exp \left(G_{\eta, \eta^{\prime}}^{\lambda}\right) \mathrm{d} \eta^{\prime}
\end{aligned}
$$

Then, we have

$$
\int_{\eta}^{\infty} \frac{1}{\sin \left(\phi^{\prime}\left(\phi, \eta, \eta^{\prime}\right)\right)} \exp \left(G_{\eta, \eta^{\prime}}^{\lambda}\right) \mathrm{d} \eta^{\prime} \leq \frac{1}{1+\lambda} \int_{-\infty}^{0} e^{z} \mathrm{~d} z \leq \frac{1}{1+\lambda},
$$

and (3.165) easily follows in this case.

In Case II, it satisfies that $\sin \phi>0$. We define that

$$
\begin{aligned}
& \mathcal{T}_{\lambda, 1}[H]=f\left(\eta^{+}, \phi_{0}\right) \exp \left(-G_{\eta, \eta^{+}}^{\lambda}\right)=\int_{\eta^{+}}^{\infty} \frac{H\left(\eta^{\prime}, R \phi^{\prime}\left(\phi, \eta, \eta^{\prime}\right)\right)}{\sin \left(\phi^{\prime}\left(\phi, \eta, \eta^{\prime}\right)\right)} \exp \left(G_{\eta, \eta^{\prime}}^{\lambda}-G_{\eta, \eta^{+}}^{\lambda}\right) \mathrm{d} \eta^{\prime}, \\
& \mathcal{T}_{\lambda, 2}[H]=\int_{\eta^{+}}^{\eta} \frac{H\left(\eta^{\prime}, \phi^{\prime}\left(\phi, \eta, \eta^{\prime}\right)\right)}{\sin \left(\phi^{\prime}\left(\phi, \eta, \eta^{\prime}\right)\right)} \exp \left(-G_{\eta, \eta^{\prime}}^{\lambda}\right) \mathrm{d} \eta^{\prime} .
\end{aligned}
$$

Since

$$
\exp \left(G_{\eta, \eta^{\prime}}^{\lambda}-G_{\eta, \eta^{+}}^{\lambda}\right) \leq \exp \left(-G_{\eta, \eta^{\prime}}^{\lambda}\right)
$$

then $\mathcal{T}_{\lambda, 1}$ can be treated as in Case III. Also, the proof of $\mathcal{T}_{\lambda, 2}$ is similar to the Case I, we omit it here. The proof of Lemma 3.9 is completed.

From Lemma 3.7, the bound of $f_{\lambda}$ in $L^{\infty}$ is independent of $\lambda$. So, let $\lambda \rightarrow 0$, we get a solution of

$$
\left\{\begin{array}{rlrl}
\sin \phi \frac{\partial f}{\partial \eta}-F(\eta) \cos \phi \frac{\partial f}{\partial \phi}+f & =H(\eta, \phi), & \eta>0 \\
f(0, \phi) & =h(\phi) & & \text { for } \sin \phi>0 \\
\lim _{\eta \rightarrow \infty} f & =f_{\infty} . & &
\end{array}\right.
$$

In this case, we denote

$$
G_{\eta, \eta^{\prime}}(\phi)=\int_{\eta^{\prime}}^{\eta} \frac{1}{\sin \left(\phi^{\prime}(\phi, \eta, \xi)\right)} \mathrm{d} \xi
$$

Then, the solution $f$ can be rewritten as

$$
f(\eta, \phi)=\mathcal{A}[h(\phi)]+\mathcal{T}[H(\eta, \phi)],
$$

where the operator $\mathcal{A}$ and $\mathcal{T}$ is defined as 
Case I. For $\sin \phi>0$ and $|E(\eta, \phi)| \leq 1$,

$$
\begin{aligned}
\mathcal{A}[h(\phi)] & =h\left(\phi^{\prime}(\phi, \eta, 0)\right) \exp \left(-G_{\eta, 0}\right) \\
\mathcal{T}[H(\eta, \phi)] & =\int_{0}^{\eta} \frac{H\left(\eta^{\prime}, \phi^{\prime}\left(\phi, \eta, \eta^{\prime}\right)\right)}{\sin \left(\phi^{\prime}\left(\phi, \eta, \eta^{\prime}\right)\right)} \exp \left(-G_{\eta, \eta^{\prime}}\right) \mathrm{d} \eta^{\prime} .
\end{aligned}
$$

Case II. For $\sin \phi>0$ and $|E(\eta, \phi)| \geq 1$,

$$
\begin{aligned}
\mathcal{A}[h(\phi)]= & 0 \\
\mathcal{T}[H(\eta, \phi)]= & f\left(\eta^{+}, \phi_{0}\right) \exp \left(-G_{\eta, \eta^{+}}\right)+\int_{\eta^{+}}^{\eta} \frac{H\left(\eta^{\prime}, \phi^{\prime}\left(\phi, \eta, \eta^{\prime}\right)\right)}{\sin \left(\phi^{\prime}\left(\phi, \eta, \eta^{\prime}\right)\right)} \exp \left(-G_{\eta, \eta^{\prime}}\right) \mathrm{d} \eta^{\prime}, \\
= & \int_{\eta^{+}}^{\infty} \frac{H\left(\eta^{\prime}, R \phi^{\prime}\left(\phi, \eta, \eta^{\prime}\right)\right)}{\sin \left(\phi^{\prime}\left(\phi, \eta, \eta^{\prime}\right)\right)} \exp \left(G_{\eta, \eta^{\prime}}-G_{\eta, \eta^{+}}\right) \mathrm{d} \eta^{\prime} \\
& +\int_{\eta^{+}}^{\eta} \frac{H\left(\eta^{\prime}, \phi^{\prime}\left(\phi, \eta, \eta^{\prime}\right)\right)}{\sin \left(\phi^{\prime}\left(\phi, \eta, \eta^{\prime}\right)\right)} \exp \left(-G_{\eta, \eta^{\prime}}\right) \mathrm{d} \eta^{\prime}
\end{aligned}
$$

Case III. For $\sin \phi<0$,

$$
\begin{aligned}
\mathcal{A}[h(\phi)] & =0 \\
\mathcal{T}[H(\eta, \phi)] & =\int_{\eta}^{\infty} \frac{H\left(\eta^{\prime}, R \phi^{\prime}\left(\phi, \eta, \eta^{\prime}\right)\right)}{\sin \left(\phi^{\prime}\left(\phi, \eta, \eta^{\prime}\right)\right)} \exp \left(G_{\eta, \eta^{\prime}}\right) \mathrm{d} \eta^{\prime} .
\end{aligned}
$$

For the estimate of the solution in $L^{\infty}$, we need the following estimate of $\mathcal{T}[H]$.

Lemma 3.10. For any $\delta>0$ there is a constant $C(\delta)>0$ independent of data such that

$$
\|\mathcal{T}[H]\|_{L^{\infty} L^{2}} \leq C(\delta)\|H\|_{L^{2} L^{2}}+\delta\|H\|_{L^{\infty} L^{\infty}}
$$

Proof. We divide the proof into several steps.

Step 1: The case of $\sin \phi>0$ and $|E(\eta, \phi)| \leq 1$.

For simplicity, we denote $\Omega_{1}=\left\{\sin \left(\phi^{\prime}\left(\phi, \eta, \eta^{\prime}\right)\right)>m\right\}$ and $\Omega_{2}=\left\{\sin \left(\phi^{\prime}\left(\phi, \eta, \eta^{\prime}\right)\right) \leq m\right\}$. Then, we consider

$$
\begin{aligned}
\int_{\sin \phi>0}|\mathcal{T}[H(\eta, \cdot)]|^{2} \mathrm{~d} \phi & =\int_{\sin \phi>0}\left(\int_{0}^{\eta} \frac{H\left(\eta^{\prime}, \phi^{\prime}\left(\phi, \eta, \eta^{\prime}\right)\right)}{\sin \left(\phi^{\prime}\left(\phi, \eta, \eta^{\prime}\right)\right)} \exp \left(-G_{\eta, \eta^{\prime}}\right) \mathrm{d} \eta^{\prime}\right)^{2} \mathrm{~d} \phi \\
& =\int\left(\int \mathbf{1}_{\Omega_{1}} \ldots\right)^{2}+\int\left(\int \mathbf{1}_{\Omega_{2}} \ldots\right)^{2} \\
& =I_{1}+I_{2}
\end{aligned}
$$

for some $m>0$. By Cauchy's inequality, we get

$$
\begin{aligned}
I_{1} & \leq \int_{\sin \phi>0}\left(\int_{0}^{\eta}\left|H\left(\eta^{\prime}, \phi^{\prime}\left(\phi, \eta, \eta^{\prime}\right)\right)\right|^{2} \mathrm{~d} \eta^{\prime}\right)\left(\int_{0}^{\eta} \mathbf{1}_{\Omega_{1}} \frac{\exp \left(-2 G_{\eta, \eta^{\prime}}\right)}{\sin ^{2}\left(\phi^{\prime}\left(\phi, \eta, \eta^{\prime}\right)\right)} \mathrm{d} \eta^{\prime}\right) \mathrm{d} \phi \\
& \leq \frac{1}{m}\|H\|_{L^{2} L^{2}}^{2} \int_{\sin \phi>0}\left(\int_{0}^{\eta} \mathbf{1}_{\Omega_{1}} \frac{\exp \left(-2 G_{\eta, \eta^{\prime}}\right)}{\sin \left(\phi^{\prime}\left(\phi, \eta, \eta^{\prime}\right)\right)} \mathrm{d} \eta^{\prime}\right) \mathrm{d} \phi \\
& \leq \frac{\pi}{m}\|H\|_{L^{2} L^{2}}^{2} .
\end{aligned}
$$

On the other hand, for $\eta^{\prime} \leq \eta$, we can directly estimate $\phi^{\prime}\left(\phi, \eta, \eta^{\prime}\right) \geq \phi$. Hence, we have the relation

$$
\sin \phi \leq \sin \left(\phi^{\prime}\left(\phi, \eta, \eta^{\prime}\right)\right)
$$


Therefore, we can directly estimate $I_{2}$ as follows:

$$
\begin{aligned}
I_{2} & \leq\|H\|_{L^{\infty} L^{\infty}}^{2} \int_{\sin \phi>0}\left(\int_{0}^{\eta} \mathbf{1}_{\Omega_{2}} \frac{1}{\sin \left(\phi^{\prime}\left(\phi, \eta, \eta^{\prime}\right)\right)} \exp \left(-G_{\eta, \eta^{\prime}}\right) \mathrm{d} \eta^{\prime}\right)^{2} \mathrm{~d} \phi \\
& \leq\|H\|_{L^{\infty} L^{\infty}}^{2} \int_{\sin \phi>0}\left(\int_{0}^{\eta} \mathbf{1}_{\Omega_{2}} \frac{1}{\sin \left(\phi^{\prime}\left(\phi, \eta, \eta^{\prime}\right)\right)} \exp \left(-G_{\eta, \eta^{\prime}}\right) \mathrm{d} \eta^{\prime}\right)^{2} \mathrm{~d} \phi \\
& =\|H\|_{L^{\infty} L^{\infty}}^{2} \int_{\Omega_{2}}\left(\int_{0}^{\eta} \frac{1}{\sin \left(\phi^{\prime}\left(\phi, \eta, \eta^{\prime}\right)\right)} \exp \left(-G_{\eta, \eta^{\prime}}\right) \mathrm{d} \eta^{\prime}\right)^{2} \mathrm{~d} \phi .
\end{aligned}
$$

Since

$$
\int_{0}^{\eta} \frac{1}{\sin \left(\phi^{\prime}\left(\phi, \eta, \eta^{\prime}\right)\right)} \exp \left(-G_{\eta, \eta^{\prime}}\right) \mathrm{d} \eta^{\prime} \leq \int_{0}^{\infty} \mathrm{e}^{-z} \mathrm{~d} z=1,
$$

we have, for $m$ sufficiently small,

$$
I_{2} \leq\|H\|_{L^{\infty} L^{\infty}}^{2} \int_{\sin \phi>0} \mathbf{1}_{\{\sin \phi \leq m\}} \mathrm{d} \phi \leq 4 m\|H\|_{L^{\infty} L^{\infty}}^{2} .
$$

Summing up (3.184) and (3.188), we deduce (3.182) for $m$ sufficiently small.

Step 2: The case of $\sin \phi<0$.

We can decompose

$$
\begin{aligned}
\int_{\sin \phi<0}|\mathcal{T} H|^{2} \mathrm{~d} \phi= & \int_{\sin \phi<0}\left(\int_{\eta}^{\infty} \frac{H\left(\eta^{\prime}, R \phi^{\prime}\left(\phi, \eta, \eta^{\prime}\right)\right)}{\sin \left(\phi^{\prime}\left(\phi, \eta, \eta^{\prime}\right)\right)} \exp \left(G_{\eta, \eta^{\prime}}\right) \mathrm{d} \eta^{\prime}\right)^{2} \mathrm{~d} \phi \\
= & \int\left(\int \mathbf{1}_{\Omega_{1}} \ldots\right)^{2}+\int\left(\int \mathbf{1}_{\Omega_{2}} \mathbf{1}_{\left\{\eta^{\prime}-\eta \geq \sigma\right\}} \cdots\right)^{2} \\
& +\int\left(\int \mathbf{1}_{\Omega_{2}} \mathbf{1}_{\left\{\eta^{\prime}-\eta \leq \sigma\right\}} \cdots\right)^{2} \\
= & I_{1}+I_{2}+I_{3},
\end{aligned}
$$

for some $m>0$ and $\sigma>0$. We can directly estimate $I_{1}$ as follows:

$$
\begin{aligned}
I_{1} & \leq \int_{\sin \phi<0}\left(\int_{\eta}^{\infty}\left|H\left(\eta^{\prime}, R \phi^{\prime}\left(\phi, \eta, \eta^{\prime}\right)\right)\right|^{2} \mathrm{~d} \eta^{\prime}\right)\left(\int_{\eta}^{\infty} \mathbf{1}_{\Omega_{1}} \frac{\exp \left(2 G_{\eta, \eta^{\prime}}\right)}{\sin ^{2}\left(\phi^{\prime}\left(\phi, \eta, \eta^{\prime}\right)\right)} \mathrm{d} \eta^{\prime}\right) \mathrm{d} \phi \\
& \leq \frac{1}{m}\|H\|_{L^{2} L^{2}}^{2} \int_{\sin \phi<0}\left(\int_{\eta}^{\infty} \mathbf{1}_{\Omega_{1}} \frac{\exp \left(2 G_{\eta, \eta^{\prime}}\right)}{\sin \left(\phi^{\prime}\left(\phi, \eta, \eta^{\prime}\right)\right)} \mathrm{d} \eta^{\prime}\right) \mathrm{d} \phi \\
& \leq \frac{\pi}{m}\|H\|_{L^{2} L^{2}}^{2} .
\end{aligned}
$$

On the other hand, for $I_{2}$ we have

$$
I_{2} \leq\|H\|_{L^{\infty} L^{\infty}}^{2} \int_{\sin \phi<0}\left(\int_{\eta}^{\infty} \mathbf{1}_{\Omega_{2}} \mathbf{1}_{\left\{\eta^{\prime}-\eta \geq \sigma\right\}} \frac{\exp \left(G_{\eta, \eta^{\prime}}\right)}{\sin \left(\phi^{\prime}\left(\phi, \eta, \eta^{\prime}\right)\right)} \mathrm{d} \eta^{\prime}\right)^{2} \mathrm{~d} \phi .
$$

Note that

$$
G_{\eta, \eta^{\prime}}^{\lambda}=\int_{\eta^{\prime}}^{\eta} \frac{1}{\sin \left(\phi^{\prime}(\phi, \eta, y)\right)} \mathrm{d} y \leq-\frac{\eta^{\prime}-\eta}{m}=-\frac{\sigma}{m}
$$

we can obtain

$$
I_{2} \leq\|H\|_{L^{\infty} L^{\infty}}^{2} \int_{\sin \phi<0}\left(\int_{\infty}^{-\sigma / m} \mathrm{e}^{z} \mathrm{~d} z\right)^{2} \mathrm{~d} \phi=4 \mathrm{e}^{-\frac{\sigma}{m}}\|H\|_{L^{\infty} L^{\infty}}^{2} .
$$

For $I_{3}$, we can estimate as follows:

$$
\begin{aligned}
I_{3} & \leq\|H\|_{L^{\infty} L^{\infty}}^{2} \int_{\sin \phi<0}\left(\int_{\eta}^{\infty} \mathbf{1}_{\Omega_{2}} \mathbf{1}_{\left\{\eta^{\prime}-\eta \leq \sigma\right\}} \frac{\exp \left(G_{\eta, \eta^{\prime}}\right)}{\sin \left(\phi^{\prime}\left(\phi, \eta, \eta^{\prime}\right)\right)} \mathrm{d} \eta^{\prime}\right)^{2} \mathrm{~d} \phi \\
& \leq\|H\|_{L^{\infty} L^{\infty}}^{2} \int_{\sin \phi<0}\left(\int_{\eta}^{\eta+\sigma} \mathbf{1}_{\Omega_{2}} \mathbf{1}_{\left\{\eta^{\prime}-\eta \leq \sigma\right\}} \frac{\exp \left(G_{\eta, \eta^{\prime}}\right)}{\sin \left(\phi^{\prime}\left(\phi, \eta, \eta^{\prime}\right)\right)} \mathrm{d} \eta^{\prime}\right)^{2} \mathrm{~d} \phi .
\end{aligned}
$$


Note that

$$
\int_{\eta}^{\infty} \frac{1}{\sin \left(\phi^{\prime}\left(\phi, \eta, \eta^{\prime}\right)\right)} \exp \left(G_{\eta, \eta^{\prime}}\right) \mathrm{d} \eta^{\prime} \leq \int_{-\infty}^{0} \mathrm{e}^{z} \mathrm{~d} z=1
$$

Then $1 \leq \alpha=\mathrm{e}^{V\left(\eta^{\prime}\right)-V(\eta)} \leq \mathrm{e}^{V(\eta+\sigma)-V(\eta)} \leq 1+4 \sigma$, and for $\left.\eta^{\prime} \in[\eta, \eta+\sigma], \sin \phi^{\prime}\left(\phi, \eta, \eta^{\prime}\right)\right)=\sin \left(\cos ^{-1}(\alpha \cos \phi)\right)$, $\sin \left(\phi^{\prime}\left(\phi, \eta, \eta^{\prime}\right)\right)<m$ lead to $\cos ^{2}\left(\phi^{\prime}\left(\phi, \eta, \eta^{\prime}\right)\right) \geq 1-m^{2}$. Thus, one has

$$
\begin{aligned}
|\sin \phi| & =\sqrt{1-\cos ^{2} \phi}=\sqrt{1-\frac{\cos ^{2} \phi^{\prime}\left(\phi, \eta, \eta^{\prime}\right)}{\alpha^{2}}} \leq \frac{\sqrt{\alpha^{2}-1+m^{2}}}{\alpha} \\
& \leq \frac{\sqrt{(1+4 \sigma)^{2}-1+m^{2}}}{\alpha} \leq \sqrt{9 \sigma+m^{2}} .
\end{aligned}
$$

Hence, we can obtain

$$
\begin{aligned}
I_{3} & \leq\|H\|_{L^{\infty} L^{\infty}}^{2} \int_{\sin \phi<0} \mathbf{1}_{\Omega_{2}} \mathrm{~d} \phi \leq\|H\|_{L^{\infty} L^{\infty}}^{2} \int_{\sin \phi<0} \mathbf{1}_{\left\{|\sin \phi| \leq \sqrt{9 \sigma+m^{2}}\right\}} \mathrm{d} \phi \\
& \leq 4 \sqrt{9 \sigma+m^{2}}\|H\|_{L^{\infty} L^{\infty}}^{2} .
\end{aligned}
$$

Step 3: The case of $\sin \phi>0$ and $|E(\eta, \phi)| \geq 1$.

$$
\begin{aligned}
\mathcal{A}[h(\phi)]= & \int_{\eta^{+}}^{\infty} \ldots \exp \left(G_{\eta, \eta^{\prime}}-G_{\eta, \eta^{+}}\right) \mathrm{d} \eta^{\prime}+\int_{\eta^{+}}^{\eta} \ldots \exp \left(-G_{\eta, \eta^{\prime}}\right) \mathrm{d} \eta^{\prime} \\
= & \int_{\eta}^{\infty} \ldots \exp \left(G_{\eta, \eta^{\prime}}-G_{\eta, \eta^{+}}\right) \mathrm{d} \eta^{\prime} \\
& +\left(\int_{\eta^{+}}^{\eta} \ldots \exp \left(G_{\eta, \eta^{\prime}}-G_{\eta, \eta^{+}}\right) \mathrm{d} \eta^{\prime}+\int_{\eta^{+}}^{\eta} \ldots \exp \left(-G_{\eta, \eta^{\prime}}\right) \mathrm{d} \eta^{\prime}\right) \\
= & I_{1}+I_{2} .
\end{aligned}
$$

Then $I_{1}$ can be treated as in Case I and $I_{2}$ as in Case III. Hence, it is already well-treated.

Summarizing all three cases, we can choose small $\sigma$ and $m$ to guarantee the relation (3.182).

3.2.3. Estimates of $\epsilon$-Milne Equation. The difference $z=f-f_{\infty}$ satisfies the following equation

$$
\left\{\begin{aligned}
\sin \phi \frac{\partial z}{\partial \eta}-F(\eta) \cos \phi \frac{\partial z}{\partial \phi}+z & =\bar{z}+S \\
z(0, \phi)=p(\phi) & =h(\phi)-f_{\infty} \text { for } \sin \phi>0 \\
\lim _{\eta \rightarrow \infty} z(\eta, \phi) & =0 .
\end{aligned}\right.
$$

Lemma 3.11. Assume (3.11) and (3.12) hold. Then there exists a constant $C$ such that the solution of equation (3.199) verifies

$$
\|z\|_{L^{\infty} L^{\infty}} \leq C\left(1+M+\frac{M}{K}+\|z\|_{L^{2} L^{2}}\right)
$$

Proof. Before giving the proof, we first show the following important inequalities for all function $l$ such that

$$
\|\bar{l}\|_{L^{2}} \leq\|l\|_{L^{2} L^{2}},\|\bar{l}\|_{L^{\infty}} \leq \frac{1}{\sqrt{2 \pi}}\|l\|_{L^{\infty} L^{2}}
$$


It could be directly derived by Cauchy's inequality as follows:

$$
\begin{aligned}
\|\bar{l}\|_{L^{2}}^{2} & =\int_{0}^{\infty}\left(\frac{1}{2 \pi}\right)^{2}\left(\int_{-\pi}^{\pi} l(\eta, \phi) \mathrm{d} \phi\right)^{2} \mathrm{~d} \eta \\
& \leq \int_{0}^{\infty} \frac{1}{2 \pi} \int_{-\pi}^{\pi} l^{2}(\eta, \phi) \mathrm{d} \phi \mathrm{d} \eta=\int_{0}^{\infty} \int_{-\pi}^{\pi} l^{2}(\eta, \phi) \mathrm{d} \phi \mathrm{d} \eta=\|l\|_{L^{2} L^{2}}^{2} \\
\|\bar{l}\|_{L^{\infty}}^{2} & =\left(\sup _{\eta} \bar{l}(\eta)\right)^{2}=\sup _{\eta}\left(\frac{1}{2 \pi} \int_{-\pi}^{\pi} l(\eta, \phi) \mathrm{d} \phi\right)^{2} \\
& \leq \frac{1}{2 \pi} \sup _{\eta} \int_{-\pi}^{\pi} l^{2}(\eta, \phi) \mathrm{d} \phi=\frac{1}{2 \pi}\|l\|_{L^{\infty} L^{2}}^{2} .
\end{aligned}
$$

Then $z$ can be rewritten along the characteristics as follows:

$$
z(\eta, \phi)=\mathcal{A}[p]+\mathcal{T}[(\bar{z}+S)(\eta, \phi)] .
$$

Then by Lemma 3.10 and (3.201), for sufficiently small $\delta$, we can show that

$$
\|\mathcal{A}[p]\|_{L^{\infty} L^{\infty}} \leq\|p\|_{L^{\infty}},
$$

and

$$
\begin{aligned}
\|\mathcal{T}[\bar{z}+S]\|_{L^{\infty} L^{2}} & \leq C(\delta)\left(\|\bar{z}\|_{L^{2}}+\|S\|_{L^{2} L^{2}}\right)+\delta\left(\|\bar{z}\|_{L^{\infty}}+\|S\|_{L^{\infty} L^{\infty}}\right) \\
& \leq C(\delta)\left(\|z\|_{L^{2} L^{2}}+\|S\|_{L^{2} L^{2}}\right)+\delta\left(\|z\|_{L^{\infty} L^{2}}+\|S\|_{L^{\infty} L^{\infty}}\right) .
\end{aligned}
$$

We can directly get

$$
\begin{aligned}
\|z\|_{L^{\infty} L^{2}} & \leq 2 \pi\|\mathcal{A}[p]\|_{L^{\infty} L^{\infty}}+\|\mathcal{T}[\bar{z}+S]\|_{L^{\infty} L^{2}} \\
& \leq 2 \pi\|p\|_{L^{\infty}}+C(\delta)\left(\|z\|_{L^{2} L^{2}}+\|S\|_{L^{2} L^{2}}\right)+\delta\left(\|z\|_{L^{\infty} L^{2}}+\|S\|_{L^{\infty} L^{\infty}}\right) .
\end{aligned}
$$

By taking $\delta=1 / 2$, there exists a constant $C$ independent of $\delta$ such that

$$
\|z\|_{L^{\infty} L^{2}} \leq C\left(\|p\|_{L^{\infty}}+\|z\|_{L^{2} L^{2}}+\|S\|_{L^{2} L^{2}}+\|S\|_{L^{\infty} L^{\infty}}\right) .
$$

Therefore, based on Lemma 3.9, (3.113), (3.201) and (3.208), we can achieve

$$
\begin{aligned}
\|z\|_{L^{\infty} L^{\infty}} & \leq\|\mathcal{A}[p]\|_{L^{\infty}}+\|\mathcal{T}[\bar{z}+S]\|_{L^{\infty} L^{\infty}} \\
& \leq C\left(\|p\|_{L^{\infty}}+\|\bar{z}\|_{L^{\infty}}+\|S\|_{L^{\infty} L^{\infty}}\right) \\
& \leq C\left(\|p\|_{L^{\infty}}+\|S\|_{L^{\infty} L^{\infty}}+\|z\|_{L^{\infty} L^{2}}\right) \\
& \leq C\left(\|p\|_{L^{\infty}}+\|S\|_{L^{\infty} L^{\infty}}+\|S\|_{L^{2} L^{2}}+\|z\|_{L^{2} L^{2}}\right) .
\end{aligned}
$$

where $C$ is independent of $\lambda$.

Since $\|p\|_{L^{\infty}},\|S\|_{L^{2} L^{2}},\|S\|_{L^{\infty} L^{\infty}}$ and $\|z\|_{L^{2} L^{2}}$ are finite, we can yield that $z$ satisfies (3.200). Then the proof of Lemma 3.11 is completed.

Combining Lemma 3.11 and Theorem 3.5 we deduce the main theorem.

Theorem 3.12. There exists a unique solution $f(\eta, \phi)$ to the $\epsilon$-Milne problem (3.8) satisfying

$$
\left\|f-f_{\infty}\right\|_{L^{\infty} L^{\infty}} \leq C\left(1+M+\frac{M}{K}\right) .
$$


3.3. Exponential Decay. In this section, we prove the spatial decay of the solution to the Milne problem.

Theorem 3.13. Assume (3.11) and (3.12) hold. For $K_{0}>0$ sufficiently small, the solution $f(\eta, \phi)$ to the $\epsilon$-Milne problem (3.8) satisfies

$$
\left\|\mathrm{e}^{K_{0} \eta}\left(f-f_{\infty}\right)\right\|_{L^{\infty} L^{\infty}} \leq C\left(1+M+\frac{M}{K}\right)
$$

Proof. Define $Z=\mathrm{e}^{K_{0} \eta} z$ for $z=f-f_{\infty}$. We divide the analysis into several steps:

Step 1:

We firstly obtain the weighted $\|\cdot\|_{L^{2} L^{2}}$ estimate of the difference $f-f_{\infty}$. That is, there is some constant $K_{0}$ is small enough such that

$$
\|Z\|_{L^{2} L^{2}}^{2}=\int_{0}^{\infty} \mathrm{e}^{2 K_{0} \eta} \int_{-\pi}^{\pi}\left(f(\eta, \phi)-f_{\infty}\right)^{2} \mathrm{~d} \phi \mathrm{d} \eta \leq C\left(1+M+\frac{M}{K}\right)^{2} .
$$

As a consequence, it also holds that

$$
\left\|\mathrm{e}^{K_{0} \eta}\left(q-q_{\infty}\right)\right\|_{L^{\infty}} \leq C\left(1+M+\frac{M}{K}\right) .
$$

The proof of (3.212): The orthogonal property (3.31) reveals

$$
\langle f, f \sin \phi\rangle_{\phi}(\eta)=\langle r, r \sin \phi\rangle_{\phi}(\eta) .
$$

Multiplying $\mathrm{e}^{2 K_{0} \eta} f$ on both sides of equation (3.8) and integrating over $\phi \in[-\pi, \pi)$, we obtain

$$
\begin{aligned}
\mathrm{e}^{2 K_{0} \eta}\langle S, f\rangle_{\phi}(\eta)= & \frac{1}{2} \frac{\mathrm{d}}{\mathrm{d} \eta}\left(\mathrm{e}^{2 K_{0} \eta}\langle r, r \sin \phi\rangle_{\phi}(\eta)\right)-\frac{1}{2} F(\eta)\left(\mathrm{e}^{2 K_{0} \eta}\langle r, r \sin \phi\rangle_{\phi}(\eta)\right) \\
& -\mathrm{e}^{2 K_{0} \eta}\left(K_{0}\langle r, r \sin \phi\rangle_{\phi}(\eta)-\langle r, r\rangle_{\phi}(\eta)\right) .
\end{aligned}
$$

For $K_{0}<1 / 2$, we have

$$
\frac{1}{2}\|r(\eta, \cdot)\|_{L^{2}}^{2} \leq-K_{0}\langle r, r \sin \phi\rangle_{\phi}(\eta)+\langle r, r\rangle_{\phi}(\eta) \leq \frac{3}{2}\|r(\eta, \cdot)\|_{L^{2}}^{2} .
$$

Let $K_{0}<\min \{1 / 2, K\}$, similar to the proof of Lemma 3.2 and Lemma 3.3. formulas (3.215) and (3.216) imply

$$
\left\|\mathrm{e}^{K_{0} \eta} r\right\|_{L^{2} L^{2}}^{2}=\int_{0}^{\infty} \mathrm{e}^{2 K_{0} \eta}\langle r, r\rangle_{\phi}(\eta) \mathrm{d} \eta \leq C\left(1+M+\frac{M}{K}\right)^{2}
$$


From (3.217) and Cauchy's inequality, noticing $q_{\infty}=f_{\infty}$, we can deduce

$$
\begin{aligned}
& \int_{0}^{\infty} \mathrm{e}^{2 K_{0} \eta}\left(\int_{-\pi}^{\pi}\left(f(\eta, \phi)-f_{\infty}\right)^{2} \mathrm{~d} \phi\right) \mathrm{d} \eta \\
& \leq \int_{0}^{\infty} \mathrm{e}^{2 K_{0} \eta}\left(\int_{-\pi}^{\pi} r^{2}(\eta, \phi) \mathrm{d} \phi\right) \mathrm{d} \eta+\int_{0}^{\infty} \mathrm{e}^{2 K_{0} \eta}\left(\int_{-\pi}^{\pi}\left(q(\eta)-q_{\infty}\right)^{2} \mathrm{~d} \phi\right) \mathrm{d} \eta \\
& \leq \int_{0}^{\infty} \mathrm{e}^{2 K_{0} \eta}\|r(\eta, \cdot)\|_{L^{2}}^{2} \mathrm{~d} \eta \\
& \quad+\int_{0}^{\infty} \mathrm{e}^{2 K_{0} \eta}\left(\int_{\eta}^{\infty}|F(y)|\|r(y, \cdot)\|_{L^{2}} \mathrm{~d} y\right)^{2} \mathrm{~d} \eta+\int_{0}^{\infty} \mathrm{e}^{2 K_{0} \eta}\left(\int_{\eta}^{\infty}\|S(y, \cdot)\|_{L^{\infty}} \mathrm{d} y\right)^{2} \mathrm{~d} \eta \\
& \leq C\left(1+M+\frac{M}{K}\right)^{2}+C\left(\int_{0}^{\infty} \mathrm{e}^{2 K_{0} \eta}\|r(\eta, \cdot)\|_{L^{2}}^{2} \mathrm{~d} \eta\right)\left(\int_{0}^{\infty} \int_{\eta}^{\infty} \mathrm{e}^{2 K_{0}(\eta-y)} F^{2}(y) \mathrm{d} y \mathrm{~d} \eta\right) \\
& \quad+\int_{0}^{\infty} \mathrm{e}^{2 K_{0} \eta}\left(\int_{\eta}^{\infty}\|S(y, \cdot)\|_{L^{\infty}} \mathrm{d} y\right)^{2} \mathrm{~d} \eta \\
& \leq C\left(1+M+\frac{M}{K}\right)^{2}+C\left(\int_{0}^{\infty} \mathrm{e}^{2 K_{0} \eta}\|r(\eta)\|_{L^{2}}^{2} \mathrm{~d} \eta\right)\left(\int_{0}^{\infty} F^{2}(y) \mathrm{d} y\right) \\
& \quad+\int_{0}^{\infty} \mathrm{e}^{2 K_{0} \eta}\left(\int_{\eta}^{\infty}\|S(y, \cdot)\|_{L^{\infty}} \mathrm{d} y\right)^{2} \mathrm{~d} \eta \\
& \leq C\left(1+M+\frac{M}{K}\right)^{2} .
\end{aligned}
$$

This completes the proof of (3.212). From the proof of Lemma 3.3, one gets that

$$
\begin{aligned}
& \left\|\mathrm{e}^{K_{0} \eta}\left(q-q_{\infty}\right)\right\|_{L^{\infty}} \\
& \leq\left\|\mathrm{e}^{2 K_{0} \eta} \int_{\eta}^{\infty}|F(y)|\right\| r(y, \cdot)\left\|_{L^{2}} \mathrm{~d} y\right\|_{L^{\infty}}+\left\|\mathrm{e}^{2 K_{0} \eta} \int_{\eta}^{\infty}\right\| S(y, \cdot)\left\|_{L^{\infty}} \mathrm{d} y\right\|_{L^{\infty}} \\
& \leq\left\|\left(\int_{\eta}^{\infty} F^{2}(y) \mathrm{d} y\right)^{\frac{1}{2}}\left(\int_{\eta}^{\infty} \mathrm{e}^{2 K_{0} y}\|r(y, \cdot)\|_{L^{2}}^{2} \mathrm{~d} y\right)^{\frac{1}{2}}\right\|_{L^{\infty}}+\left\|\int_{\eta}^{\infty} \mathrm{e}^{2 K_{0} y}\right\| S(y, \cdot)\left\|_{L^{\infty}} \mathrm{d} y\right\|_{L^{\infty}} \\
& \leq C\left(1+M+\frac{M}{K}\right) .
\end{aligned}
$$

This shows (3.213) when $\bar{S}=0$. Noting that all the auxiliary functions constructed in Lemma 3.4 satisfy the estimates (3.212) and (3.213), then we can extend above $L^{2}$ estimates to the general $S$ case by the method introduced in Lemma 3.4 .

Step 2:

We consider the decay rate of $f-f_{\infty}$ w.r.t. the spatial variable $\eta$. By a similar argument as before, we can easily show that there exists $K_{0}$ small enough such that

$$
\left\|\mathrm{e}^{K_{0} \eta} \mathcal{A}[p]\right\|_{L^{\infty}} \leq\|p\|_{L^{\infty}} .
$$

and the integral operator $\mathcal{T}$ satisfies

$$
\left\|\mathrm{e}^{K_{0} \eta} \mathcal{T}[H]\right\|_{L^{\infty} L^{\infty}} \leq C\|H\|_{L^{\infty} L^{\infty}},
$$

where $C$ is a universal constant independent $H$. With these estimates and Lemma 3.10 we will obtain

$$
\|Z\|_{L^{\infty} L^{\infty}} \leq C\left(1+M+\frac{M}{K}+\|Z\|_{L^{2} L^{2}}\right) .
$$

Proof of (3.221): $Z$ satisfies the equation

$$
\left\{\begin{aligned}
\sin \phi \frac{\partial Z}{\partial \eta}+F(\eta) \cos \phi \frac{\partial Z}{\partial \phi}+Z & =\bar{Z}+\mathrm{e}^{K_{0} \eta} S+K_{0} \sin \phi Z \\
Z(0, \phi) & =p(\phi)=h(\phi)-f_{\infty} \text { for } \sin \phi>0 .
\end{aligned}\right.
$$


Since we know

$$
Z=\mathcal{A}[p]+\mathcal{T}\left[\bar{Z}+\mathrm{e}^{K_{0} \eta} S+K_{0} \sin \phi Z\right]
$$

then by Lemma 3.10, (3.201), we can show

$$
\begin{aligned}
& \left\|\mathcal{T}\left[\bar{Z}+\mathrm{e}^{K_{0} \eta} S+K_{0} \sin \phi Z\right]\right\|_{L^{\infty} L^{2}} \\
& \leq C(\delta)\left(\left\|\bar{Z}+\mathrm{e}^{K_{0} \eta} S+K_{0} Z\right\|_{L^{2} L^{2}}\right)+\delta\left(\left\|\bar{Z}+\mathrm{e}^{K_{0} \eta} S+K_{0} Z\right\|_{L^{\infty} L^{\infty}}\right) \\
& \leq C(\delta)\left(\|Z\|_{L^{2} L^{2}}+\left\|\mathrm{e}^{K_{0} \eta} S\right\|_{L^{2} L^{2}}+K_{0}\|Z\|_{L^{2} L^{2}}\right) \\
& +\delta\left(\|Z\|_{L^{\infty} L^{2}}+\left\|\mathrm{e}^{K_{0} \eta} S\right\|_{L^{\infty} L^{\infty}}+K_{0}\|Z\|_{L^{\infty} L^{\infty}}\right) .
\end{aligned}
$$

Therefore, based on Lemma 3.8 and (3.206), we can directly estimate

$$
\begin{aligned}
\|Z\|_{L^{\infty} L^{2}} \leq & 2 \pi\|\mathcal{A}[p]\|_{L^{\infty}}+\left\|\mathcal{T}\left[\bar{Z}+\mathrm{e}^{K_{0} \eta} S+K_{0} \sin \phi Z\right]\right\|_{L^{\infty} L^{2}} \\
\leq & 2 \pi\|p\|_{L^{\infty}}+C(\delta)\left(\|Z\|_{L^{2} L^{2}}+\left\|\mathrm{e}^{K_{0} \eta} S\right\|_{L^{2} L^{2}}\right) \\
& +\delta\left(\|Z\|_{L^{\infty} L^{2}}+\left\|\mathrm{e}^{K_{0} \eta} S\right\|_{L^{\infty} L^{\infty}}+K_{0}\|Z\|_{L^{\infty} L^{\infty}}\right) .
\end{aligned}
$$

By taking $\delta=1 / 2$, we obtain

$$
\|Z\|_{L^{\infty} L^{2}} \leq C\left(\|p\|_{L^{\infty}}+\left\|\mathrm{e}^{K_{0} \eta} S\right\|_{L^{2} L^{2}}+\left\|\mathrm{e}^{K_{0} \eta} S\right\|_{L^{\infty} L^{\infty}}+\|Z\|_{L^{2} L^{2}}+K_{0}\|Z\|_{L^{\infty} L^{\infty}}\right) .
$$

Then based on Lemma 3.8, Lemma 3.9, Lemma 3.10 and (3.213), we can deduce

$$
\begin{aligned}
\|Z\|_{L^{\infty} L^{\infty}} & \leq\left\|\mathrm{e}^{K_{0} \eta} \mathcal{A}[p]\right\|_{L^{\infty} L^{\infty}}+\left\|\mathcal{T}\left[\bar{Z}+e^{K_{0} \eta} S+K_{0} Z\right]\right\|_{L^{\infty} L^{\infty}} \\
& \leq\|p\|_{L^{\infty}}+\|\bar{Z}\|_{L^{\infty} L^{\infty}}+\left\|\mathrm{e}^{K_{0} \eta} S\right\|_{L^{\infty} L^{\infty}}+K_{0}\|Z\|_{L^{\infty} L^{\infty}} \\
& \leq\|p\|_{L^{\infty}}+\|Z\|_{L^{\infty} L^{2}}+\left\|U^{\epsilon} e^{K_{0} \eta} S\right\|_{L^{\infty} L^{\infty}}+K_{0}\|Z\|_{L^{\infty} L^{\infty}} \\
& \leq C\left(1+M+\frac{M}{K}\right)+C\left(\|Z\|_{L^{2} L^{2}}+K_{0}\|Z\|_{L^{\infty} L^{\infty}}\right) .
\end{aligned}
$$

Taking $K_{0}$ sufficiently small, this completes the proof of (3.221).

Combining (3.212) and (3.221), we deduce (3.211).

\subsection{Maximum Principle.}

Theorem 3.14. The solution $f(\eta, \phi)$ to the $\epsilon$-Milne problem (3.8) with $S=0$ satisfies the maximum principle, i.e.

$$
\min _{\sin \phi>0} h(\phi) \leq f(\eta, \phi) \leq \max _{\sin \phi>0} h(\phi)
$$

Proof. We claim it is sufficient to show $f(\eta, \phi) \leq 0$ whenever $h(\phi) \leq 0$. Suppose this claim is justified. Denote $m=\min _{\sin \phi>0} h(\phi)$ and $M=\max _{\sin \phi>0} h(\phi)$. Then $f^{1}=f-M$ satisfies the equation

$$
\left\{\begin{aligned}
\sin \phi \frac{\partial f^{1}}{\partial \eta}-F(\eta) \cos \phi \frac{\partial f^{1}}{\partial \phi}+f^{1}-\bar{f}^{1} & =0 \\
f^{1}(0, \phi) & =h(\phi)-M \text { for } \sin \phi>0 \\
\lim _{\eta \rightarrow \infty} f^{1}(\eta, \phi) & =f_{\infty}^{1} .
\end{aligned}\right.
$$

Hence, $h-M \leq 0$ implies $f^{1} \leq 0$ which is actually $f \leq M$. On the other hand, $f^{2}=m-f$ satisfies the equation

$$
\left\{\begin{aligned}
\sin \phi \frac{\partial f^{2}}{\partial \eta}-F(\eta) \cos \phi \frac{\partial f^{2}}{\partial \phi}+f^{2}-\bar{f}^{2} & =0 \\
f^{2}(0, \phi) & =m-h(\phi) \text { for } \sin \phi>0 \\
\lim _{\eta \rightarrow \infty} f^{2}(\eta, \phi) & =f_{\infty}^{2}
\end{aligned}\right.
$$


Thus, $m-h \leq 0$ implies $f^{2} \leq 0$ which further leads to $f \geq m$. Therefore, the maximum principle is established.

We now prove if $h(\phi) \leq 0$, we have $f(\eta, \phi) \leq 0$. We divide the proof into several steps:

Step 1: Penalized $\epsilon$-Milne problem in a finite slab.

Assuming $h(\phi) \leq 0$, we then consider the penalized Milne problem for $f_{\lambda}^{L}(\eta, \phi)$ in the finite slab $(\eta, \phi) \in$ $[0, L] \times[-\pi, \pi)$

$$
\left\{\begin{aligned}
\lambda f_{\lambda}^{L}+\sin \phi \frac{\partial f_{\lambda}^{L}}{\partial \eta}-F(\eta) \cos \phi \frac{\partial f_{\lambda}^{L}}{\partial \phi}+f_{\lambda}^{L}-\bar{f}_{\lambda}^{L} & =0 \\
f_{\lambda}^{L}(0, \phi) & =h(\phi) \text { for } \sin \phi<0, \\
f_{\lambda}^{L}(L, \phi) & =f_{\lambda}^{L}(L, R \phi) .
\end{aligned}\right.
$$

In order to construct the solution of (3.230), we iteratively define the sequence $\left\{f_{m}^{L}\right\}_{m=1}^{\infty}$ as $f_{0}^{L}=0$ and

$$
\left\{\begin{aligned}
\lambda f_{m}^{L}+\sin \phi \frac{\partial f_{m}^{L}}{\partial \eta}-F(\eta) \cos \phi \frac{\partial f_{m}^{L}}{\partial \phi}+f_{m}^{L}-\bar{f}_{m-1}^{L} & =0 \\
f_{m}^{L}(0, \phi) & =h(\phi) \text { for } \sin \phi<0 \\
f_{m}^{L}(L, \phi) & =f_{m}^{L}(L, R \phi) .
\end{aligned}\right.
$$

Along the characteristics, it is easy to see we always have $f_{m}^{L}<0$. In the proof of Lemma 3.2, we have shown $f_{m}^{L}$ converges strongly in $L^{\infty}([0, L] \times[-\pi, \pi))$ to $f_{\lambda}^{L}$ which satisfies (3.230). Also, $f_{\lambda}^{L}$ satisfies

$$
\left\|f_{\lambda}^{L}\right\|_{L^{\infty} L^{\infty}} \leq \frac{1+\lambda}{\lambda}\|h\|_{L^{\infty}} .
$$

Naturally, we obtain $f_{\lambda}^{L} \in L^{2}([0, L] \times[-\pi, \pi))$ and $f_{\lambda}^{L} \leq 0$.

Step 2: $\epsilon$-Milne problem in a finite slab.

Consider the Milne problem for $f^{L}(\eta, \phi)$ in a finite slab $(\eta, \phi) \in[0, L] \times[-\pi, \pi)$

$$
\left\{\begin{aligned}
\sin \phi \frac{\partial f^{L}}{\partial \eta}-F(\eta) \cos \phi \frac{\partial f^{L}}{\partial \phi}+f^{L}-\bar{f}^{L} & =0, \\
f^{L}(0, \phi) & =h(\phi) \text { for } \sin \phi<0, \\
f^{L}(L, \phi) & =f^{L}(L, R \phi) .
\end{aligned}\right.
$$

In the proof of Lemma 3.2, we have shown $f_{\lambda}^{L}$ is uniformly bounded in $L^{2}([0, L) \times[-\pi, \pi))$ with respect to $\lambda$, which implies we can take weakly convergent subsequence $f_{\lambda}^{L} \rightarrow f^{L}$ as $\lambda \rightarrow 0$ with $f^{L} \in L^{2}([0, L] \times[-\pi, \pi))$. Naturally, we have $f^{L}(\eta, \phi) \leq 0$.

Step 3: $\epsilon$-Milne problem in an infinite slab.

Finally, in the proof of Lemma 3.3. by taking $L \rightarrow \infty$, we have

$$
f^{L} \rightarrow f \text { in } L_{l o c}^{2}([0, L) \times[-\pi, \pi),
$$

where $f$ satisfies (3.8). Certainly, we have $f(\eta, \phi) \leq 0$. This justifies the claim in Step 1. Hence, we complete the proof.

Remark 3.15. Note that when $F=0$, then all the previous proofs can be recovered and Theorem 3.12, Theorem 3.13 and Theorem 3.14 still hold. Hence, we can deduce the well-posedness, decay and maximum principle of the classical Milne problem

$$
\left\{\begin{aligned}
\sin \phi \frac{\partial f}{\partial \eta}+f-\bar{f} & =S(\eta, \phi) \\
f(0, \phi) & =h(\phi) \text { for } \sin \phi>0 \\
\lim _{\eta \rightarrow \infty} f(\eta, \phi) & =f_{\infty}
\end{aligned}\right.
$$




\section{Remainder Estimate}

In this section, we consider the remainder estimate of the equation

$$
\left\{\begin{aligned}
\epsilon \vec{w} \cdot \nabla_{x} u+u-\bar{u} & =f(\vec{x}, \vec{w}) \text { in } \Omega, \\
u\left(\vec{x}_{0}, \vec{w}\right) & =g\left(\vec{x}_{0}, \vec{w}\right) \text { for } \vec{x}_{0} \in \partial \Omega \text { and } \vec{w} \cdot \vec{n}<0 .
\end{aligned}\right.
$$

Let the measurable functions be defined on a.e. $\Omega \times \mathcal{S}^{1}$. The Lebesgue spaces of measurable functions on $\Omega \times \mathcal{S}^{1}$ are denoted by $L^{p}\left(\Omega \times \mathcal{S}^{1}\right), 1 \leq p \leq \infty$. These spaces are complete with respect to the norm

$$
\|f\|_{L^{p}\left(\Omega \times \mathcal{S}^{1}\right)}=\left(\int_{\Omega} \int_{\mathcal{S}^{1}}|f(\vec{x}, \vec{w})|^{p} \mathrm{~d} \vec{w} \mathrm{~d} \vec{x}\right)^{1 / p}
$$

and $L^{2}\left(\Omega \times \mathcal{S}^{1}\right)$ is a Hilbert space with scalar product

$$
(f, g)=(f, g)_{L^{2}\left(\Omega \times \mathcal{S}^{1}\right)}=\int_{\Omega} \int_{\mathcal{S}^{1}} f g \mathrm{~d} \vec{w} \mathrm{~d} \vec{x} .
$$

In particular, the $L^{2}$ and $L^{\infty}$ norms are defined as follows

$$
\begin{aligned}
\|f\|_{L^{2}\left(\Omega \times \mathcal{S}^{1}\right)} & =\left(\int_{\Omega} \int_{\mathcal{S}^{1}}|f(\vec{x}, \vec{w})|^{2} \mathrm{~d} \vec{w} \mathrm{~d} \vec{x}\right)^{1 / 2}, \\
\|f\|_{L^{\infty}\left(\Omega \times \mathcal{S}^{1}\right)} & =\sup _{(\vec{x}, \vec{w}) \in \Omega \times \mathcal{S}^{1}}|f(\vec{x}, \vec{w})| .
\end{aligned}
$$

Let $\mathrm{d} s$ be the Lebesgue measure on $\partial \Omega$, then we consider the trace spaces $L^{p}(\Gamma)$ for $p \geq 1$ endowed with the norm

$$
\begin{aligned}
\|f\|_{L^{p}(\Gamma)} & =\left(\int_{\Gamma}|f(\vec{x}, \vec{w})|^{p} \mathrm{~d} \xi\right)^{1 / 2}, \\
\|f\|_{L^{p}\left(\Gamma^{ \pm}\right)} & =\left(\int_{\Gamma^{ \pm}}|f(\vec{x}, \vec{w})|^{p} \mathrm{~d} \xi\right)^{1 / 2} .
\end{aligned}
$$

where $\mathrm{d} \xi=|\vec{w} \cdot \vec{n}(x)| \mathrm{d} s \mathrm{~d} \vec{w}$. At the same time, the $L^{\infty}$ norm of the function on the boundary is defined as

$$
\begin{aligned}
\|f\|_{L^{\infty}(\Gamma)} & =\sup _{(\vec{x}, \vec{w}) \in \Gamma}|f(\vec{x}, \vec{w})|, \\
\|f\|_{L^{\infty}\left(\Gamma^{ \pm}\right)} & =\sup _{(\vec{x}, \vec{w}) \in \Gamma^{ \pm}}|f(\vec{x}, \vec{w})| .
\end{aligned}
$$

In what follows, we would show the well-posedness of the solution to (4.1).

4.1. Preliminaries. In order to show the $L^{2}$ and $L^{\infty}$ estimates of the equation (4.1), we start with some preparations with the penalized neutron transport equation.

Lemma 4.1. Assume $f(\vec{x}, \vec{w}) \in L^{\infty}\left(\Omega \times \mathcal{S}^{1}\right)$ and $g\left(\vec{x}_{0}, \vec{w}\right) \in L^{\infty}(\Gamma)$ with $\vec{x}_{0} \in \partial \Omega$. Then, for any $\lambda>0$ and $\epsilon$, there exists a solution $u_{\lambda}(\vec{x}, \vec{w}) \in L^{\infty}\left(\Omega \times \mathcal{S}^{1}\right)$ of the penalized transport equation

$$
\left\{\begin{aligned}
\lambda u_{\lambda}+\epsilon \vec{w} \cdot \nabla_{x} u_{\lambda}+u_{\lambda} & =f(\vec{x}, \vec{w}) \text { in } \Omega \times \mathcal{S}^{1}, \\
u_{\lambda} & =g \text { on } \partial \Omega \times \mathcal{S}^{1}, \vec{w} \cdot \vec{n}<0
\end{aligned}\right.
$$

which satisfies the following bound

$$
\left\|u_{\lambda}\right\|_{L^{\infty}\left(\Omega \times \mathcal{S}^{1}\right)} \leq\|g\|_{L^{\infty}\left(\Gamma^{-}\right)}+\|f\|_{L^{\infty}\left(\Omega \times \mathcal{S}^{1}\right)} .
$$

Proof. The characteristics $(X(s), W(s))$ of the equation (4.17) which goes through $(\vec{x}, \vec{w})$ is defined by

$$
\left\{\begin{array}{l}
\frac{\mathrm{d} X(s)}{\mathrm{d} s}=\epsilon W(s), \frac{\mathrm{d} W(s)}{\mathrm{d} s}=0 \\
(X(0), W(0))=(\vec{x}, \vec{w}) .
\end{array}\right.
$$

which implies

$$
X(s)=\vec{x}+(\epsilon \vec{w}) s, W(s)=\vec{w}
$$


Hence, we can rewrite the equation (4.17) along the characteristics as

$$
u_{\lambda}(\vec{x}, \vec{w})=g\left(\vec{x}-\epsilon t_{b} \vec{w}, \vec{w}\right) e^{-(1+\lambda) t_{b}}+\int_{0}^{t_{b}} f\left(\vec{x}-\epsilon\left(t_{b}-s\right) \vec{w}, \vec{w}\right) e^{-(1+\lambda)\left(t_{b}-s\right)} \mathrm{d} s,
$$

where the backward exit time $t_{b}$ is defined as

$$
t_{b}(\vec{x}, \vec{w})=\inf \left\{t \geq 0:(\vec{x}-\epsilon t \vec{w}, \vec{w}) \in \Gamma^{-}\right\} .
$$

Then, since $t_{b} \geq 0$, it gives the following estimate

$$
\begin{aligned}
\left|u_{\lambda}(\vec{x}, \vec{w})\right| & \leq e^{-(1+\lambda) t_{b}}\|g\|_{L^{\infty}\left(\Gamma^{-}\right)}+\frac{1-e^{(1+\lambda) t_{b}}}{1+\lambda}\|f\|_{L^{\infty}\left(\Omega \times \mathcal{S}^{1}\right)} \\
& \leq\|g\|_{L^{\infty}\left(\Gamma^{-}\right)}+\|f\|_{L^{\infty}\left(\Omega \times \mathcal{S}^{1}\right)} .
\end{aligned}
$$

Since $u_{\lambda}$ can be explicitly traced back to the boundary data, the existence of the solution to the equation (4.17) naturally follows from the above estimate.

Lemma 4.2. Assume $f(\vec{x}, \vec{w}) \in L^{\infty}\left(\Omega \times \mathcal{S}^{1}\right)$ and $g(\vec{x}, \vec{w}) \in L^{\infty}(\Gamma)$ with $\vec{x} \in \partial \Omega$. Then, for any $\lambda>0$ and $\epsilon$, there exists a solution $u_{\lambda}(\vec{x}, \vec{w}) \in L^{\infty}\left(\Omega \times \mathcal{S}^{1}\right)$ of the penalized transport equation

$$
\left\{\begin{aligned}
\lambda u_{\lambda}+\epsilon \vec{w} \cdot \nabla_{x} u_{\lambda}+u_{\lambda}-\bar{u}_{\lambda} & =f(\vec{x}, \vec{w}) \text { in } \Omega \times \mathcal{S}^{1}, \\
u_{\lambda} & =g \text { on } \partial \Omega \times \mathcal{S}^{1}, \vec{w} \cdot \vec{n}<0,
\end{aligned}\right.
$$

which satisfies the following bound

$$
\left\|u_{\lambda}\right\|_{L^{\infty}\left(\Omega \times \mathcal{S}^{1}\right)} \leq \frac{1+\lambda}{\lambda}\left(\|f\|_{L^{\infty}\left(\Omega \times \mathcal{S}^{1}\right)}+\|g\|_{L^{\infty}\left(\Gamma^{-}\right)}\right) .
$$

Proof. We define an approximating sequence $\left\{u_{\lambda}^{k}\right\}_{k=0}^{\infty}$, where $u_{\lambda}^{0}=0$ and

$$
\left\{\begin{aligned}
\lambda u_{\lambda}^{k}+\epsilon \vec{w} \cdot \nabla_{x} u_{\lambda}^{k}+u_{\lambda}^{k} & =\bar{u}_{\lambda}^{k-1}+f(\vec{x}, \vec{w}) \text { in } \Omega \times \mathcal{S}^{1}, \\
u_{\lambda}^{k} & =g \text { on } \partial \Omega \times \mathcal{S}^{1} \text { and } \vec{w} \cdot \vec{n}<0 .
\end{aligned}\right.
$$

By Lemma 4.1, $u_{\lambda}^{1}$ is well-defined and

$$
\left\|u_{\lambda}^{1}\right\|_{L^{\infty}\left(\Omega \times \mathcal{S}^{1}\right)}<\|g\|_{L^{\infty}\left(\Gamma^{-}\right)}+\|f\|_{L^{\infty}\left(\Omega \times \mathcal{S}^{1}\right)} .
$$

We define the difference $v^{k}=u_{\lambda}^{k}-u_{\lambda}^{k-1}$ for $k \geq 1$. Recall (1.2) for $\bar{v}^{k}$, then $v^{k}$ satisfies

$$
\left\{\begin{aligned}
\lambda v^{k}+\epsilon \vec{w} \cdot \nabla_{x} v^{k}+v^{k} & =\bar{v}^{k-1} \text { in } \Omega \times \mathcal{S}^{1}, \\
v_{\lambda}^{k} & =0 \text { on } \partial \Omega \times \mathcal{S}^{1} \text { and } \vec{w} \cdot \vec{n}<0 .
\end{aligned}\right.
$$

Since $\left\|\bar{v}^{k}\right\|_{L^{\infty}\left(\Omega \times \mathcal{S}^{1}\right)} \leq\left\|v^{k}\right\|_{L^{\infty}\left(\Omega \times \mathcal{S}^{1}\right)}$, we naturally have

$$
\left\|v^{k+1}\right\|_{L^{\infty}\left(\Omega \times \mathcal{S}^{1}\right)} \leq \frac{1}{1+\lambda}\left\|\bar{v}^{k}\right\|_{L^{\infty}\left(\Omega \times \mathcal{S}^{1}\right)} \leq \frac{1}{1+\lambda}\left\|v^{k}\right\|_{L^{\infty}\left(\Omega \times \mathcal{S}^{1}\right)} .
$$

Thus, $\left(u_{\lambda}^{k}\right)_{k=0}^{\infty}$ is a contraction series for $\lambda>0$. Since $v^{1}=u_{\lambda}^{1}$, by combining (4.20) and (4.22), it easily deduces

$$
\left\|u^{k}\right\|_{L^{\infty}\left(\Omega \times \mathcal{S}^{1}\right)} \leq \sum_{k=1}^{\infty}\left\|v^{k}\right\|_{L^{\infty}\left(\Omega \times \mathcal{S}^{1}\right)} \leq \frac{1+\lambda}{\lambda}\left(\|g\|_{L^{\infty}\left(\Gamma^{-}\right)}+\|f\|_{L^{\infty}\left(\Omega \times \mathcal{S}^{1}\right)}\right) .
$$

Let $k \rightarrow \infty$, we get the existence of the solution to (4.17). This completes the proof of Lemma 4.2,

From (4.17), the bound of the solution depends on $\lambda$. Then we can not get the solution of the equation by letting $\lambda$ tends to zero. So, we need to show a uniform estimate of the solution to the penalized neutron transport equation (4.17) with respect to $\lambda$. 
4.2. Uniform $L^{2}$ Estimate. We recall the following Green's Identity, which could be found in 2, Chapter $9]$ and $[3$.

Lemma 4.3. (Green's Identity) Assume $f(\vec{x}, \vec{w}), g(\vec{x}, \vec{w}) \in L^{2}\left(\Omega \times \mathcal{S}^{1}\right)$ and $\vec{w} \cdot \nabla_{x} f, \vec{w} \cdot \nabla_{x} g \in L^{2}\left(\Omega \times \mathcal{S}^{1}\right)$ with $f, g \in L^{2}(\Gamma)$. Then

$$
\iint_{\Omega \times \mathcal{S}^{1}}\left(\left(\vec{w} \cdot \nabla_{x} f\right) g+\left(\vec{w} \cdot \nabla_{x} g\right) f\right) \mathrm{d} \vec{x} \mathrm{~d} \vec{w}=\int_{\Gamma} f g \mathrm{~d} \gamma
$$

where $\mathrm{d} \gamma=(\vec{w} \cdot \vec{n}) \mathrm{d}$ s on the boundary.

We firstly give the uniform $L^{2}$ estimate of the hydrodynamic part of the solution.

Lemma 4.4. The solution $u_{\lambda}$ to the equation (4.17) satisfies the uniform estimate

$$
\epsilon\left\|\bar{u}_{\lambda}\right\|_{L^{2}\left(\Omega \times \mathcal{S}^{1}\right)} \leq C(\Omega)\left(\left\|u_{\lambda}-\bar{u}_{\lambda}\right\|_{L^{2}\left(\Omega \times \mathcal{S}^{1}\right)}+\|f\|_{L^{2}\left(\Omega \times \mathcal{S}^{1}\right)}+\epsilon\left\|u_{\lambda}\right\|_{L^{2}\left(\Gamma^{+}\right)}+\epsilon\|g\|_{L^{2}\left(\Gamma^{-}\right)}\right),
$$

for $0 \leq \lambda \leq 1$ and $0<\epsilon \leq 1$.

Proof. From Lemma 4.2, it is nature that $u_{\lambda} \in L^{2}\left(\Omega \times \mathcal{S}^{1}\right)$ as well as $u_{\lambda} \in L^{2}(\Gamma)$. It follows that $\vec{w} \cdot \nabla u_{\lambda} \in$ $L^{2}\left(\Omega \times \mathcal{S}^{1}\right)$ from the equation (4.17). Then, for any $\phi \in L^{2}\left(\Omega \times \mathcal{S}^{1}\right)$ satisfying $\vec{w} \cdot \nabla_{x} \phi \in L^{2}\left(\Omega \times \mathcal{S}^{1}\right)$ and $\phi \in L^{2}(\Gamma)$, we have

$$
\lambda \iint_{\Omega \times \mathcal{S}^{1}} u_{\lambda} \phi+\epsilon \int_{\Gamma} u_{\lambda} \phi \mathrm{d} \gamma-\epsilon \iint_{\Omega \times \mathcal{S}^{1}} u_{\lambda}\left(\vec{w} \cdot \nabla_{x} \phi\right)+\iint_{\Omega \times \mathcal{S}^{1}}\left(u_{\lambda}-\bar{u}_{\lambda}\right) \phi=\iint_{\Omega \times \mathcal{S}^{1}} f \phi .
$$

Similar to [13, it needs to choose a particular test function $\phi$. We define $\zeta(\vec{x})$ on $\Omega$ satisfying

$$
\left\{\begin{aligned}
\Delta \zeta & =\bar{u}_{\lambda} \text { in } \Omega, \\
\zeta & =0 \text { on } \partial \Omega .
\end{aligned}\right.
$$

Based on the standard elliptic estimate, we have

$$
\|\zeta\|_{H^{2}(\Omega)} \leq C(\Omega)\left\|\bar{u}_{\lambda}\right\|_{L^{2}(\Omega)} .
$$

The test function is defined as

$$
\phi=-\vec{w} \cdot \nabla_{x} \zeta
$$

Naturally, we have

$$
\|\phi\|_{H^{1}(\Omega)}+\|\phi\|_{L^{\infty}(\Omega)} \leq C\|\zeta\|_{H^{2}(\Omega)} \leq C(\Omega)\left\|\bar{u}_{\lambda}\right\|_{L^{2}(\Omega)} .
$$

We can decompose

$$
-\epsilon \iint_{\Omega \times \mathcal{S}^{1}}\left(\vec{w} \cdot \nabla_{x} \phi\right) u_{\lambda}=-\epsilon \iint_{\Omega \times \mathcal{S}^{1}}\left(\vec{w} \cdot \nabla_{x} \phi\right) \bar{u}_{\lambda}-\epsilon \iint_{\Omega \times \mathcal{S}^{1}}\left(\vec{w} \cdot \nabla_{x} \phi\right)\left(u_{\lambda}-\bar{u}_{\lambda}\right) .
$$

For the first part, we have

$$
\begin{aligned}
-\epsilon \iint_{\Omega \times \mathcal{S}^{1}}\left(\vec{w} \cdot \nabla_{x} \phi\right) \bar{u}_{\lambda} & =\epsilon \iint_{\Omega \times \mathcal{S}^{1}} \bar{u}_{\lambda}\left(w_{1}\left(w_{1} \partial_{11} \zeta+w_{2} \partial_{12} \zeta\right)+w_{2}\left(w_{1} \partial_{12} \zeta+w_{2} \partial_{22} \zeta\right)\right) \\
& =\epsilon \iint_{\Omega \times \mathcal{S}^{1}} \bar{u}_{\lambda}\left(w_{1}^{2} \partial_{11} \zeta+2 w_{1} w_{2} \partial_{12}+w_{2}^{2} \partial_{22} \zeta\right) \\
& =\epsilon \pi \int_{\Omega} \bar{u}_{\lambda}\left(\partial_{11} \zeta+\partial_{22} \zeta\right) \\
& =\epsilon \pi\left\|\bar{u}_{\lambda}\right\|_{L^{2}(\Omega)}^{2} \\
& =\frac{1}{2} \epsilon\left\|\bar{u}_{\lambda}\right\|_{L^{2}\left(\Omega \times \mathcal{S}^{1}\right)}^{2} .
\end{aligned}
$$

On the other hand, Hölder's inequality and the elliptic estimate imply

$$
\begin{aligned}
\left|\epsilon \iint_{\Omega \times \mathcal{S}^{1}}\left(\vec{w} \cdot \nabla_{x} \phi\right)\left(u_{\lambda}-\bar{u}_{\lambda}\right)\right| & \leq C(\Omega) \epsilon\left\|u_{\lambda}-\bar{u}_{\lambda}\right\|_{L^{2}\left(\Omega \times \mathcal{S}^{1}\right)}\|\zeta\|_{H^{2}(\Omega)} \\
& \leq C(\Omega) \epsilon\left\|u_{\lambda}-\bar{u}_{\lambda}\right\|_{L^{2}\left(\Omega \times \mathcal{S}^{1}\right)}\left\|\bar{u}_{\lambda}\right\|_{L^{2}\left(\Omega \times \mathcal{S}^{1}\right)}
\end{aligned}
$$


Based on (4.28), (4.30), the boundary condition of the penalized neutron transport equation (4.17), the trace theorem, Hölder's inequality and the elliptic estimate, we have

$$
\begin{aligned}
&\left|\epsilon \int_{\Gamma} u_{\lambda} \phi \mathrm{d} \gamma\right| \leq \epsilon\left(\int_{\Gamma}\left|u_{\lambda}\right|^{2} \mathrm{~d} \gamma\right)^{1 / 2}\left(\int_{\Gamma}|\phi|^{2} \mathrm{~d} \gamma\right)^{1 / 2} \\
& \leq C(\Omega) \epsilon\left(\left\|u_{\lambda}\right\|_{L^{2}\left(\Gamma^{+}\right)}+\|g\|_{L^{2}\left(\Gamma^{-}\right)}\right)\left\|\bar{u}_{\lambda}\right\|_{L^{2}\left(\Omega \times \mathcal{S}^{1}\right)} \\
& \lambda\left|\iint_{\Omega \times \mathcal{S}^{1}} u_{\lambda} \phi\right|=\lambda\left|\iint_{\Omega \times \mathcal{S}^{1}} \bar{u}_{\lambda} \phi+\lambda \iint_{\Omega \times \mathcal{S}^{1}}\left(u_{\lambda}-\bar{u}_{\lambda}\right) \phi\right| \\
&= \lambda \iint_{\Omega \times \mathcal{S}^{1}}\left|\left(u_{\lambda}-\bar{u}_{\lambda}\right) \phi\right| \\
& \leq C(\Omega) \lambda\left\|u_{\lambda}-\bar{u}_{\lambda}\right\|_{L^{2}\left(\Omega \times \mathcal{S}^{1}\right)}\left\|\bar{u}_{\lambda}\right\|_{L^{2}\left(\Omega \times \mathcal{S}^{1}\right)}, \\
&\left|\iint_{\Omega \times \mathcal{S}^{1}}\left(u_{\lambda}-\bar{u}_{\lambda}\right) \phi\right| \leq C(\Omega)\left\|u_{\lambda}-\bar{u}_{\lambda}\right\|_{L^{2}\left(\Omega \times \mathcal{S}^{1}\right)}\left\|\bar{u}_{\lambda}\right\|_{L^{2}\left(\Omega \times \mathcal{S}^{1}\right)} \\
&\left|\iint_{\Omega \times \mathcal{S}^{1}} f \phi\right| \leq C(\Omega)\left\|\bar{u}_{\lambda}\right\|_{L^{2}\left(\Omega \times \mathcal{S}^{1}\right)}\|f\|_{L^{2}\left(\Omega \times \mathcal{S}^{1}\right)} .
\end{aligned}
$$

Collecting terms in (4.32), (4.33), (4.34), (4.35), (4.36) and (4.37), we obtain

$$
\begin{aligned}
\epsilon\left\|\bar{u}_{\lambda}\right\|_{L^{2}\left(\Omega \times \mathcal{S}^{1}\right)} \leq & C(\Omega)\left((1+\epsilon+\lambda)\left\|u_{\lambda}-\bar{u}_{\lambda}\right\|_{L^{2}\left(\Omega \times \mathcal{S}^{1}\right)}\right. \\
& \left.+\epsilon\left\|u_{\lambda}\right\|_{L^{2}\left(\Gamma^{+}\right)}+\|f\|_{L^{2}\left(\Omega \times \mathcal{S}^{1}\right)}+\epsilon\|g\|_{L^{2}\left(\Gamma^{-}\right)}\right),
\end{aligned}
$$

So we get the desired uniform estimate with respect to $0 \leq \lambda \leq 1$.

Theorem 4.5. Assume $f(\vec{x}, \vec{w}) \in L^{2}\left(\Omega \times \mathcal{S}^{1}\right)$ and $g\left(\vec{x}_{0}, \vec{w}\right) \in L^{2}\left(\Gamma^{-}\right)$. Then for the steady neutron transport equation 4.1), there exists a unique solution $u(\vec{x}, \vec{w}) \in L^{2}\left(\Omega \times \mathcal{S}^{1}\right)$ satisfying

$$
\|u\|_{L^{2}\left(\Omega \times \mathcal{S}^{1}\right)} \leq C(\Omega)\left(\frac{1}{\epsilon^{2}}\|f\|_{L^{2}\left(\Omega \times \mathcal{S}^{1}\right)}+\frac{1}{\epsilon^{1 / 2}}\|g\|_{L^{2}\left(\Gamma^{-}\right)}\right) .
$$

Proof. In the weak formulation (4.26), we may take the test function $\phi=u_{\lambda}$ to get the energy estimate

$$
\lambda\left\|u_{\lambda}\right\|_{L^{2}\left(\Omega \times \mathcal{S}^{1}\right)}^{2}+\frac{1}{2} \epsilon \int_{\Gamma}\left|u_{\lambda}\right|^{2} \mathrm{~d} \gamma+\left\|u_{\lambda}-\bar{u}_{\lambda}\right\|_{L^{2}\left(\Omega \times \mathcal{S}^{1}\right)}^{2}=\iint_{\Omega \times \mathcal{S}^{1}} f u_{\lambda} .
$$

Hence, this naturally implies

$$
\frac{1}{2} \epsilon\left\|u_{\lambda}\right\|_{L^{2}\left(\Gamma^{+}\right)}^{2}+\left\|u_{\lambda}-\bar{u}_{\lambda}\right\|_{L^{2}\left(\Omega \times \mathcal{S}^{1}\right)}^{2} \leq\left|\iint_{\Omega \times \mathcal{S}^{1}} f u_{\lambda}\right|+\frac{1}{2} \epsilon\|g\|_{L^{2}\left(\Gamma^{-}\right)}^{2} .
$$

On the other hand, we can square on both sides of (4.25) to obtain

$$
\epsilon^{2}\left\|\bar{u}_{\lambda}\right\|_{L^{2}\left(\Omega \times \mathcal{S}^{1}\right)}^{2} \leq C(\Omega)\left(\left\|u_{\lambda}-\bar{u}_{\lambda}\right\|_{L^{2}\left(\Omega \times \mathcal{S}^{1}\right)}^{2}+\|f\|_{L^{2}\left(\Omega \times \mathcal{S}^{1}\right)}^{2}+\epsilon^{2}\left\|u_{\lambda}\right\|_{L^{2}\left(\Gamma^{+}\right)}^{2}+\epsilon^{2}\|g\|_{L^{2}\left(\Gamma^{-}\right)}^{2}\right) .
$$

Multiplying a sufficiently small constant on both sides of (4.42) and adding it to (4.41) to absorb $\left\|u_{\lambda}\right\|_{L^{2}\left(\Gamma^{+}\right)}^{2}$ and $\left\|u_{\lambda}-\bar{u}_{\lambda}\right\|_{L^{2}\left(\Omega \times \mathcal{S}^{1}\right)}^{2}$, we deduce

$$
\begin{aligned}
\epsilon\left\|u_{\lambda}\right\|_{L^{2}\left(\Gamma^{+}\right)}^{2}+ & \epsilon^{2}\left\|\bar{u}_{\lambda}\right\|_{L^{2}\left(\Omega \times \mathcal{S}^{1}\right)}^{2}+\left\|u_{\lambda}-\bar{u}_{\lambda}\right\|_{L^{2}\left(\Omega \times \mathcal{S}^{1}\right)}^{2} \\
& \leq C(\Omega)\left(\|f\|_{L^{2}\left(\Omega \times \mathcal{S}^{1}\right)}^{2}+\left|\iint_{\Omega \times \mathcal{S}^{1}} f u_{\lambda}\right|+\epsilon\|g\|_{L^{2}\left(\Gamma^{-}\right)}^{2}\right) .
\end{aligned}
$$

Hence, we have

$$
\epsilon\left\|u_{\lambda}\right\|_{L^{2}\left(\Gamma^{+}\right)}^{2}+\epsilon^{2}\left\|u_{\lambda}\right\|_{L^{2}\left(\Omega \times \mathcal{S}^{1}\right)}^{2} \leq C(\Omega)\left(\|f\|_{L^{2}\left(\Omega \times \mathcal{S}^{1}\right)}^{2}+\left|\iint_{\Omega \times \mathcal{S}^{1}} f u_{\lambda}\right|+\epsilon\|g\|_{L^{2}\left(\Gamma^{-}\right)}^{2}\right) .
$$


A simple application of Cauchy's inequality leads to

$$
\iint_{\Omega \times \mathcal{S}^{1}} f u_{\lambda} \leq \frac{1}{4 C \epsilon^{2}}\|f\|_{L^{2}\left(\Omega \times \mathcal{S}^{1}\right)}^{2}+C \epsilon^{2}\left\|u_{\lambda}\right\|_{L^{2}\left(\Omega \times \mathcal{S}^{1}\right)}^{2} .
$$

Taking $C$ sufficiently small, we can divide (4.44) by $\epsilon^{2}$ to obtain

$$
\frac{1}{\epsilon}\left\|u_{\lambda}\right\|_{L^{2}\left(\Gamma^{+}\right)}^{2}+\left\|u_{\lambda}\right\|_{L^{2}\left(\Omega \times \mathcal{S}^{2}\right)}^{2} \leq C(\Omega)\left(\frac{1}{\epsilon^{4}}\|f\|_{L^{2}\left(\Omega \times \mathcal{S}^{2}\right)}^{2}+\frac{1}{\epsilon}\|g\|_{L^{2}\left(\Gamma^{-}\right)}^{2}\right) .
$$

Since above estimate does not depend on $\lambda$, it gives a uniform estimate for the penalized neutron transport equation (4.17). Thus, we can extract a weakly convergent subsequence $u_{\lambda} \rightarrow u$ as $\lambda \rightarrow 0$. The weak lower semi-continuity of norms $\|\cdot\|_{L^{2}\left(\Omega \times \mathcal{S}^{2}\right)}$ and $\|\cdot\|_{L^{2}\left(\Gamma^{+}\right)}$implies $u$ also satisfies the estimate (4.46). Hence, in the weak formulation (4.26), we can take $\lambda \rightarrow 0$ to deduce that $u$ satisfies equation (4.1). Also $u_{\lambda}-u$ satisfies the equation

$$
\left\{\begin{aligned}
\epsilon \vec{w} \cdot \nabla_{x}\left(u_{\lambda}-u\right)+\left(u_{\lambda}-u\right)-\left(\bar{u}_{\lambda}-\bar{u}\right) & =-\lambda u_{\lambda} \text { in } \Omega \\
\left(u_{\lambda}-u\right)\left(\vec{x}_{0}, \vec{w}\right) & =0 \text { for } \vec{x}_{0} \in \partial \Omega \text { and } \vec{w} \cdot \vec{n}<0 .
\end{aligned}\right.
$$

By a similar argument as above, we can achieve

$$
\left\|u_{\lambda}-u\right\|_{L^{2}\left(\Omega \times \mathcal{S}^{2}\right)}^{2} \leq C(\Omega)\left(\frac{\lambda}{\epsilon^{4}}\left\|u_{\lambda}\right\|_{L^{2}\left(\Omega \times \mathcal{S}^{2}\right)}^{2}\right) .
$$

When $\lambda \rightarrow 0$, the right-hand side approaches zero, which implies the convergence is actually in the strong sense. The uniqueness easily follows from the energy estimates.

\section{3. $L^{\infty}$ Estimate.}

Theorem 4.6. Assume $f(\vec{x}, \vec{w}) \in L^{\infty}\left(\Omega \times \mathcal{S}^{1}\right)$ and $g\left(\vec{x}_{0}, \vec{w}\right) \in L^{\infty}\left(\Gamma^{-}\right)$. Then for the neutron transport equation (4.1), there exists a unique solution $u(\vec{x}, \vec{w}) \in L^{\infty}\left(\Omega \times \mathcal{S}^{1}\right)$ satisfying

$$
\|u\|_{L^{\infty}\left(\Omega \times \mathcal{S}^{1}\right)} \leq C(\Omega)\left(\frac{1}{\epsilon^{3}}\|f\|_{L^{\infty}\left(\Omega \times \mathcal{S}^{1}\right)}+\frac{1}{\epsilon^{3 / 2}}\|g\|_{L^{\infty}\left(\Gamma^{-}\right)}\right) .
$$

Proof. We divide the proof into several steps to bootstrap an $L^{2}$ solution to an $L^{\infty}$ solution:

Step 1: Double Duhamel's iterations.

The characteristics of the equation (4.1) is given by (4.12). Hence, we can rewrite the equation (4.1) along the characteristics as

$$
\begin{aligned}
u(\vec{x}, \vec{w})= & g\left(\vec{x}-\epsilon t_{b} \vec{w}, \vec{w}\right) \mathrm{e}^{-t_{b}}+\int_{0}^{t_{b}} f\left(\vec{x}-\epsilon\left(t_{b}-s\right) \vec{w}, \vec{w}\right) \mathrm{e}^{-\left(t_{b}-s\right)} \mathrm{d} s \\
& +\frac{1}{2 \pi} \int_{0}^{t_{b}}\left(\int_{\mathcal{S}^{1}} u\left(\vec{x}-\epsilon\left(t_{b}-s\right) \vec{w}, \vec{w}_{t}\right) \mathrm{d} \vec{w}_{t}\right) \mathrm{e}^{-\left(t_{b}-s\right)} \mathrm{d} s .
\end{aligned}
$$

where the backward exit time $t_{b}$ is defined as (4.15). Note we have replaced $\bar{u}$ by the integral of $u$ over the dummy velocity variable $\vec{w}_{t}$. For the last term in this formulation, we apply the Duhamel's principle again to $u\left(\vec{x}-\epsilon\left(t_{b}-s\right) \vec{w}, \vec{w}_{t}\right)$ and obtain

$$
\begin{aligned}
u(\vec{x}, \vec{w})= & g\left(\vec{x}-\epsilon t_{b} \vec{w}, \vec{w}\right) \mathrm{e}^{-t_{b}}+\int_{0}^{t_{b}} f\left(\vec{x}-\epsilon\left(t_{b}-s\right) \vec{w}, \vec{w}\right) \mathrm{e}^{-\left(t_{b}-s\right)} \mathrm{d} s \\
& +\frac{1}{2 \pi} \int_{0}^{t_{b}} \int_{\mathcal{S}^{1}} g\left(\vec{x}-\epsilon\left(t_{b}-s\right) \vec{w}-\epsilon s_{b} \vec{w}_{t}, \vec{w}_{t}\right) \mathrm{e}^{-s_{b}} \mathrm{~d} \vec{w}_{t} \mathrm{e}^{-\left(t_{b}-s\right)} \mathrm{d} s \\
& +\frac{1}{2 \pi} \int_{0}^{t_{b}} \int_{\mathcal{S}^{1}}\left(\int_{0}^{s_{b}} f\left(\vec{x}-\epsilon\left(t_{b}-s\right) \vec{w}-\epsilon\left(s_{b}-r\right) \vec{w}_{t}, \vec{w}_{t}\right) \mathrm{e}^{-\left(s_{b}-r\right)} \mathrm{d} r\right) \mathrm{d} \vec{w}_{t} \mathrm{e}^{-\left(t_{b}-s\right)} \mathrm{d} s \\
& +\frac{1}{(2 \pi)^{2}} \int_{0}^{t_{b}} \int_{\mathcal{S}^{1}} \mathrm{e}^{-\left(t_{b}-s\right)}\left(\int_{0}^{s_{b}} \int_{\mathcal{S}^{1}} \mathrm{e}^{-\left(s_{b}-r\right)} u\left(\vec{x}-\epsilon\left(t_{b}-s\right) \vec{w}-\epsilon\left(s_{b}-r\right) \vec{w}_{t}, \vec{w}_{s}\right) \mathrm{d} \vec{w}_{s} \mathrm{~d} r\right) \mathrm{d} \vec{w}_{t} \mathrm{~d} s
\end{aligned}
$$

where we introduce another dummy velocity variable $\vec{w}_{s}$ and

$$
s_{b}\left(\vec{x}, \vec{w}, s, \vec{w}_{t}\right)=\inf \left\{r \geq 0:\left(\vec{x}-\epsilon\left(t_{b}-s\right) \vec{w}-\epsilon r \vec{w}_{t}, \vec{w}_{t}\right) \in \Gamma^{-}\right\} .
$$


Step 2: Estimates of all but the last term in (4.51).

We can directly estimate as follows:

$$
\begin{gathered}
\left|g\left(\vec{x}-\epsilon t_{b} \vec{w}, \vec{w}\right) e^{-t_{b}}\right| \leq\|g\|_{L^{\infty}\left(\Gamma^{-}\right)} \\
\left|\frac{1}{2 \pi} \int_{0}^{t_{b}} \int_{\mathcal{S}^{1}} g\left(\vec{x}-\epsilon\left(t_{b}-s\right) \vec{w}-\epsilon s_{b} \vec{w}_{t}, \vec{w}_{t}\right) \mathrm{e}^{-s_{b}} \mathrm{~d} \vec{w}_{t} \mathrm{e}^{-\left(t_{b}-s\right)} \mathrm{d} s\right| \leq\|g\|_{L^{\infty}\left(\Gamma^{-}\right)} \\
\left|\int_{0}^{t_{b}} f\left(\vec{x}-\epsilon\left(t_{b}-s\right) \vec{w}, \vec{w}\right) \mathrm{e}^{-\left(t_{b}-s\right)} \mathrm{d} s\right| \leq\|f\|_{L^{\infty}\left(\Omega \times \mathcal{S}^{1}\right)} \\
\left|\frac{1}{2 \pi} \int_{0}^{t_{b}} \int_{\mathcal{S}^{1}}\left(\int_{0}^{s_{b}} f\left(\vec{x}-\epsilon\left(t_{b}-s\right) \vec{w}-\epsilon\left(s_{b}-r\right) \vec{w}_{t}, \vec{w}_{t}\right) \mathrm{e}^{-\left(s_{b}-r\right)} \mathrm{d} r\right) \mathrm{d} \vec{w}_{t} \mathrm{e}^{-\left(t_{b}-s\right)} \mathrm{d} s\right| \leq\|f\|_{L^{\infty}\left(\Omega \times \mathcal{S}^{1}\right)}
\end{gathered}
$$

Step 3: Estimates of the last term in (4.51).

Now we decompose the last term in (4.51) as

$$
\int_{0}^{t_{b}} \int_{\mathcal{S}^{1}} \int_{0}^{s_{b}} \int_{\mathcal{S}^{1}}=\int_{0}^{t_{b}} \int_{\mathcal{S}^{1}} \int_{s_{b}-r \leq \delta} \int_{\mathcal{S}^{1}}+\int_{0}^{t_{b}} \int_{\mathcal{S}^{1}} \int_{s_{b}-r \geq \delta} \int_{\mathcal{S}^{1}}=I_{1}+I_{2},
$$

for some $\delta>0$. We can estimate $I_{1}$ directly as

$$
\left|I_{1}\right| \leq \int_{0}^{t_{b}} \mathrm{e}^{-\left(t_{b}-s\right)}\left(\int_{\max \left\{0, s_{b}-\delta\right\}}^{s_{b}}\|u\|_{L^{\infty}\left(\Omega \times \mathcal{S}^{1}\right)} \mathrm{d} r\right) \mathrm{d} s \leq \delta\|u\|_{L^{\infty}\left(\Omega \times \mathcal{S}^{1}\right)} .
$$

Then we can bound $I_{2}$ as

$$
I_{2} \leq \int_{0}^{t_{b}} \int_{\mathcal{S}^{1}} \int_{0}^{\max \left\{0, s_{b}-\delta\right\}} \int_{\mathcal{S}^{1}}\left|u\left(\vec{x}-\epsilon\left(t_{b}-s\right) \vec{w}-\epsilon\left(s_{b}-r\right) \vec{w}_{t}, \vec{w}_{s}\right)\right| \mathrm{e}^{-\left(t_{b}-s\right)} \mathrm{e}^{-\left(s_{b}-r\right)} \mathrm{d} \vec{w}_{s} \mathrm{~d} r \mathrm{~d} \vec{w}_{t} \mathrm{~d} s .
$$

By the definition of $t_{b}$ and $s_{b}$, we always have $\vec{x}-\epsilon\left(t_{b}-s\right) \vec{w}-\epsilon\left(s_{b}-r\right) \vec{w}_{t} \in \bar{\Omega}$. Hence, we may interchange the order of integration and apply Hölder's inequality to obtain

$$
\begin{aligned}
\left|I_{2}\right| \leq & \left(\int_{0}^{t_{b}} \int_{\mathcal{S}^{1}} \int_{0}^{\max \left\{0, s_{b}-\delta\right\}} \int_{\mathcal{S}^{1}} \mathrm{e}^{-\left(t_{b}-s\right)} \mathrm{e}^{-\left(s_{b}-r\right)} \mathrm{d} \vec{w}_{s} \mathrm{~d} r \mathrm{~d} \vec{w}_{t} \mathrm{~d} s\right)^{1 / 2} \\
& \left(\int_{0}^{t_{b}} \int_{\mathcal{S}^{1}} \int_{0}^{\max \left\{0, s_{b}-\delta\right\}} \mathbf{1}_{\vec{x}-\epsilon\left(t_{b}-s\right) \vec{w}-\epsilon\left(s_{b}-r\right) \vec{w}_{t} \epsilon \bar{\Omega}}\right. \\
& \left.|u|^{2}\left(\vec{x}-\epsilon\left(t_{b}-s\right) \vec{w}-\epsilon\left(s_{b}-r\right) \vec{w}_{t}, \vec{w}_{s}\right) \mathrm{e}^{-\left(t_{b}-s\right)} \mathrm{e}^{-\left(s_{b}-r\right)} \mathrm{d} \vec{w}_{s} \mathrm{~d} r \mathrm{~d} \vec{w}_{t} \mathrm{~d} s\right)^{1 / 2} \\
\leq & \left(\int_{0}^{t_{b}} \int_{\mathcal{S}^{1}} \int_{0}^{\max \left\{0, s_{b}-\delta\right\}} \mathbf{1}_{\vec{x}-\epsilon\left(t_{b}-s\right) \vec{w}-\epsilon\left(s_{b}-r\right) \vec{w}_{t} \epsilon \bar{\Omega}}\right. \\
& \left.|u|^{2}\left(\vec{x}-\epsilon\left(t_{b}-s\right) \vec{w}-\epsilon\left(s_{b}-r\right) \vec{w}_{t}, \vec{w}_{s}\right) \mathrm{e}^{-\left(t_{b}-s\right)} \mathrm{e}^{-\left(s_{b}-r\right)} \mathrm{d} \vec{w}_{s} \mathrm{~d} r \mathrm{~d} \vec{w}_{t} \mathrm{~d} s\right)^{1 / 2} .
\end{aligned}
$$

We may write it in a new variable $\psi$ as $\vec{w}_{t}=(\cos \psi, \sin \psi)$. By the change of the variable $[-\pi, \pi] \times \mathbb{R}^{\prime} \rightarrow$ $\Omega:(\psi, r) \rightarrow\left(y_{1}, y_{2}\right)=\vec{x}-\epsilon\left(t_{b}-s\right) \vec{w}-\epsilon\left(s_{b}-r\right) \vec{w}_{t}$, i.e.

$$
\left\{\begin{array}{l}
y_{1}=x_{1}-\epsilon\left(t_{b}-s\right) w_{1}-\epsilon\left(s_{b}-r\right) \cos \psi \\
y_{2}=x_{2}-\epsilon\left(t_{b}-s\right) w_{2}-\epsilon\left(s_{b}-r\right) \sin \psi
\end{array}\right.
$$

Therefore, for $s_{b}-r \geq \delta$, we can directly compute the Jacobian

$$
\left|\frac{\partial\left(y_{1}, y_{2}\right)}{\partial(\psi, r)}\right|=\left\|\begin{array}{rr}
\epsilon\left(s_{b}-r\right) \sin \psi & \epsilon \cos \psi \\
-\epsilon\left(s_{b}-r\right) \cos \psi & \epsilon \sin \psi
\end{array}\right\|=\epsilon^{2}\left(s_{b}-r\right) \geq \epsilon^{2} \delta .
$$


Then we may further utilize Cauchy's inequality and the $L^{2}$ estimate of $u$ in Theorem 4.5 to obtain we get

$$
\begin{aligned}
\left|I_{2}\right| & \leq \frac{C}{\epsilon \sqrt{\delta}}\|u\|_{L^{2}\left(\Omega \times \mathcal{S}^{1}\right)} \\
& \leq \frac{C(\Omega)}{\sqrt{\delta}}\left(\frac{1}{\epsilon^{3}}\|f\|_{L^{2}\left(\Omega \times \mathcal{S}^{1}\right)}+\frac{1}{\epsilon^{3 / 2}}\|g\|_{L^{2}\left(\Gamma^{-}\right)}\right) \\
& \leq \frac{C(\Omega)}{\sqrt{\delta}}\left(\frac{1}{\epsilon^{3}}\|f\|_{L^{\infty}\left(\Omega \times \mathcal{S}^{1}\right)}+\frac{1}{\epsilon^{3 / 2}}\|g\|_{L^{\infty}\left(\Gamma^{-}\right)}\right) .
\end{aligned}
$$

In summary, collecting (4.53), (4.54), (4.55), (4.56), (4.58) and (4.63), for fixed $0<\delta<1$, we have

$$
|u(\vec{x}, \vec{w})| \leq \delta\|u\|_{L^{\infty}\left(\Omega \times \mathcal{S}^{1}\right)}+\frac{C(\Omega)}{\sqrt{\delta}}\left(\frac{1}{\epsilon^{3}}\|f\|_{L^{\infty}\left(\Omega \times \mathcal{S}^{1}\right)}+\frac{1}{\epsilon^{3 / 2}}\|g\|_{L^{\infty}\left(\Gamma^{-}\right)}\right) .
$$

Taking supremum of $u$ over all $(\vec{x}, \vec{w})$ and absorbing $\|u\|_{L^{\infty}\left(\Omega \times \mathcal{S}^{1}\right)}$, for fixed $0<\delta \leq 1 / 2$, we get

$$
\|u\|_{L^{\infty}\left(\Omega \times \mathcal{S}^{1}\right)} \leq C(\Omega)\left(\frac{1}{\epsilon^{3}}\|f\|_{L^{\infty}\left(\Omega \times \mathcal{S}^{1}\right)}+\frac{1}{\epsilon^{3 / 2}}\|g\|_{L^{\infty}\left(\Gamma^{-}\right)}\right) .
$$

\section{Diffusive Limit}

The proof of 1.1 We divide the proof into several steps:

Step 1: Remainder definitions.

We may rewrite the asymptotic expansion as follows:

$$
u^{\epsilon} \sim \sum_{k=0}^{\infty} \epsilon^{k} U_{k}^{\epsilon}+\sum_{k=0}^{\infty} \epsilon^{k} \mathscr{U}_{+, k}^{\epsilon}+\sum_{k=0}^{\infty} \epsilon^{k} \mathscr{U}_{-, k}^{\epsilon}
$$

The remainder can be defined as

$$
R_{N}=u^{\epsilon}-\sum_{k=0}^{N} \epsilon^{k} U_{k}^{\epsilon}-\sum_{k=0}^{\infty} \epsilon^{k} \mathscr{U}_{+, k}^{\epsilon}-\sum_{k=0}^{\infty} \epsilon^{k} \mathscr{U}_{-, k}^{\epsilon}=u^{\epsilon}-Q_{N}-\mathscr{Q}_{+, N}-\mathscr{Q}_{-, N},
$$

where

$$
\begin{gathered}
Q_{N}=\sum_{k=0}^{N} \epsilon^{k} U_{k}^{\epsilon}, \\
\mathscr{Q}_{+, N}=\sum_{k=0}^{N} \epsilon^{k} \mathscr{U}_{+, k}^{\epsilon}, \\
\mathscr{Q}_{-, N}=\sum_{k=0}^{N} \epsilon^{k} \mathscr{U}_{-, k}^{\epsilon} .
\end{gathered}
$$

Noting the equation (2.42) is equivalent to the equation (1.1), we write $\mathcal{L}$ to denote the neutron transport operator as follows:

$$
\begin{aligned}
\mathcal{L} u & =\epsilon \vec{w} \cdot \nabla_{x} u+u-\bar{u} \\
& = \pm \sin \phi \frac{\partial u}{\partial \eta_{ \pm}}-\frac{\epsilon}{R_{ \pm} \mp \epsilon \eta_{ \pm}} \cos \phi\left(\frac{\partial u}{\partial \phi}+\frac{\partial u}{\partial \theta}\right)+u-\bar{u} .
\end{aligned}
$$

Step 2: Estimates of $\mathcal{L} Q_{N}$.

The interior contribution can be estimated as

$$
\mathcal{L} Q_{0}=\epsilon \vec{w} \cdot \nabla_{x} Q_{0}+Q_{0}-\bar{Q}_{0}=\epsilon \vec{w} \cdot \nabla_{x} U_{0}^{\epsilon}+\left(U_{0}^{\epsilon}-\bar{U}_{0}^{\epsilon}\right)=\epsilon \vec{w} \cdot \nabla_{x} U_{0}^{\epsilon} .
$$

We have

$$
\left|\epsilon \vec{w} \cdot \nabla_{x} U_{0}^{\epsilon}\right| \leq C \epsilon\left|\nabla_{x} U_{0}^{\epsilon}\right| \leq C \epsilon
$$


This implies

$$
\left|\mathcal{L} Q_{0}\right| \leq C \epsilon .
$$

Similarly, for higher order term, we can estimate

$$
\mathcal{L} Q_{N}=\epsilon \vec{w} \cdot \nabla_{x} Q_{N}+Q_{N}-\bar{Q}_{N}=\epsilon^{N+1} \vec{w} \cdot \nabla_{x} U_{N}^{\epsilon} .
$$

We have

$$
\left|\epsilon^{N+1} \vec{w} \cdot \nabla_{x} U_{N}^{\epsilon}\right| \leq C \epsilon^{N+1}\left|\nabla_{x} U_{N}^{\epsilon}\right| \leq C \epsilon^{N+1} .
$$

This implies

$$
\left|\mathcal{L} Q_{N}\right| \leq C \epsilon^{N+1}
$$

Step 3: Estimates of $\mathcal{L} \mathscr{Q}_{ \pm, N}$.

The boundary layer solution is $\mathscr{U}_{k}^{\epsilon}=\left(f_{ \pm, k}^{\epsilon}-f_{ \pm, k}^{\epsilon}(\infty)\right) \cdot \psi_{0}=\mathscr{V}_{ \pm, k} \psi_{0}$ where $f_{ \pm, k}^{\epsilon}\left(\eta_{ \pm}, \theta, \phi\right)$ solves the $\epsilon$-Milne problem and $\mathscr{V}_{ \pm, k}=f_{ \pm, k}^{\epsilon}-f_{ \pm, k}^{\epsilon}(\infty)$. Notice $\psi_{0} \psi=\psi_{0}$, so the boundary layer contribution can be estimated as

$$
\begin{aligned}
& \mathcal{L}_{ \pm, 0} \\
= & \pm \sin \phi \frac{\partial \mathscr{Q}_{ \pm, 0}}{\partial \eta_{ \pm}}-\frac{\epsilon}{R_{ \pm} \mp \epsilon \eta_{ \pm}} \cos \phi\left(\frac{\partial \mathscr{Q}_{ \pm, 0}}{\partial \phi}+\frac{\partial \mathscr{Q}_{ \pm, 0}}{\partial \theta}\right)+\mathscr{Q}_{ \pm, 0}-\overline{\mathscr{Q}}_{ \pm, 0} \\
= & \pm \sin \phi\left(\psi_{0} \frac{\partial \mathscr{V}_{ \pm, 0}}{\partial \eta_{ \pm}}+\mathscr{V}_{ \pm, 0} \frac{\partial \psi_{0}}{\partial \eta_{ \pm}}\right)-\frac{\psi_{0} \epsilon}{R_{ \pm} \mp \epsilon \eta_{ \pm}} \cos \phi\left(\frac{\partial \mathscr{V}_{ \pm, 0}}{\partial \phi}+\frac{\partial \mathscr{V}_{ \pm, 0}}{\partial \theta}\right)+\psi_{0} \mathscr{V}_{ \pm, 0}-\psi_{0} \overline{\mathscr{V}}_{ \pm, 0} \\
= & \pm \sin \phi\left(\psi_{0} \frac{\partial \mathscr{V}_{ \pm, 0}}{\partial \eta_{ \pm}}+\mathscr{V}_{ \pm, 0} \frac{\partial \psi_{0}}{\partial \eta_{ \pm}}\right)-\frac{\psi_{0} \psi \epsilon}{R_{ \pm} \mp \epsilon \eta_{ \pm}} \cos \phi\left(\frac{\partial \mathscr{V}_{ \pm, 0}}{\partial \phi}+\frac{\partial \mathscr{V}_{ \pm, 0}}{\partial \theta}\right)+\psi_{0} \mathscr{V}_{ \pm, 0}-\psi_{0} \overline{\mathscr{V}}_{ \pm, 0} \\
= & \psi_{0}\left( \pm \sin \phi \frac{\partial \mathscr{V}_{ \pm, 0}}{\partial \eta_{ \pm}}-\frac{\epsilon \psi}{R_{ \pm} \mp \epsilon \eta_{ \pm}} \cos \phi \frac{\partial \mathscr{V}_{ \pm, 0}}{\partial \phi}+\mathscr{V}_{ \pm, 0}-\overline{\mathscr{V}}_{ \pm, 0}\right) \pm \sin \phi \frac{\partial \psi_{0}}{\partial \eta_{ \pm}} \mathscr{V}_{ \pm, 0}-\frac{\psi_{0} \epsilon}{R_{ \pm} \mp \epsilon \eta_{ \pm}} \cos \phi \frac{\partial \mathscr{V}_{ \pm, 0}}{\partial \theta} \\
= & \pm \sin \phi \frac{\partial \psi_{0}}{\partial \eta_{ \pm}} \mathscr{V}_{ \pm, 0}-\frac{\psi_{0} \epsilon}{R_{ \pm} \mp \epsilon \eta_{ \pm}} \cos \phi \frac{\partial \mathscr{V}_{ \pm, 0}}{\partial \theta} .
\end{aligned}
$$

Since $\psi_{0}=1$ when $\eta_{ \pm} \leq 1 /(4 \epsilon)\left(R_{+}-R_{-}\right)$, the effective region of $\partial_{\eta} \psi_{0}$ is $\eta \geq 1 /(4 \epsilon)\left(R_{+}-R_{-}\right)$which is further and further from the origin as $\epsilon \rightarrow 0$. By Theorem 4.13 in [13] and Theorem 3.13, the first term in (5.13) can be controlled as

$$
\left| \pm \sin \phi \frac{\partial \psi_{0}}{\partial \eta_{ \pm}} \mathscr{V}_{ \pm, 0}\right| \leq C e^{-\frac{K_{0}}{\epsilon}} \leq C \epsilon .
$$

For the second term in (5.13), we have

$$
\left|-\frac{\psi_{0} \epsilon}{R_{ \pm} \mp \epsilon \eta_{ \pm}} \cos \phi \frac{\partial \mathscr{V}_{ \pm, 0}}{\partial \theta}\right| \leq C \epsilon\left|\frac{\partial \mathscr{V}_{ \pm, 0}}{\partial \theta}\right| \leq C \epsilon .
$$

This implies

$$
\left|\mathcal{L}_{ \pm, 0}\right| \leq C \epsilon .
$$

Similarly, for higher order term, we can estimate

$$
\begin{aligned}
\mathcal{L}_{\mathscr{Q}_{ \pm, N}} & =\sin \phi \frac{\partial \mathscr{Q}_{ \pm, N}}{\partial \eta_{ \pm}}-\frac{\epsilon}{R_{ \pm} \mp \epsilon \eta_{ \pm}} \cos \phi\left(\frac{\partial \mathscr{Q}_{ \pm, N}}{\partial \phi}+\frac{\partial \mathscr{Q}_{ \pm, N}}{\partial \theta}\right)+\mathscr{Q}_{ \pm, N}-\overline{\mathscr{Q}}_{ \pm, N} \\
& =\sum_{i=0}^{N} \epsilon^{i} \sin \phi \frac{\partial \psi_{0}}{\partial \eta_{ \pm}} \mathscr{V}_{ \pm, i}-\frac{\psi_{0} \epsilon^{N+1}}{R_{ \pm} \mp \epsilon \eta_{ \pm}} \cos \phi \frac{\partial \mathscr{V}_{ \pm, N}}{\partial \theta} .
\end{aligned}
$$

Away from the origin, the first term in (5.17) can be controlled as

$$
\left|\sum_{i=0}^{N} \epsilon^{i} \sin \phi \frac{\partial \psi_{0}}{\partial \eta_{ \pm}} \mathscr{V}_{ \pm, i}\right| \leq C e^{-\frac{K_{0}}{\epsilon}} \leq C \epsilon^{N+1} .
$$


For the second term in (5.17), we have

$$
\left|-\frac{\psi_{0} \epsilon^{N+1}}{R_{ \pm} \mp \epsilon \eta_{ \pm}} \cos \phi \frac{\partial \mathscr{V}_{ \pm, N}}{\partial \theta}\right| \leq C \epsilon^{N+1}\left|\frac{\partial \mathscr{V}_{ \pm, N}}{\partial \theta}\right| \leq C \epsilon^{N+1}
$$

This implies

$$
\left|\mathcal{L}_{N}\right| \leq C \epsilon^{N+1}
$$

Step 4: Diffusive limit.

Proof of (1.12). In summary, since $\mathcal{L} u^{\epsilon}=0$, collecting (5.2), (5.12) and (5.20), we can prove

$$
\left|\mathcal{L} R_{N}\right| \leq C \epsilon^{N+1} \text {. }
$$

Consider the asymptotic expansion to $N=3$, then the remainder $R_{3}$ satisfies the equation

$$
\left\{\begin{aligned}
\epsilon \vec{w} \cdot \nabla_{x} R_{3}+R_{3}-\bar{R}_{3} & =\mathcal{L} R_{3} \text { for } \vec{x} \in \Omega, \\
R_{3}\left(\vec{x}_{0}, \vec{w}\right) & =0 \text { for } \vec{w} \cdot \vec{n}<0 \text { and } \vec{x}_{0} \in \partial \Omega .
\end{aligned}\right.
$$

By Theorem 3.6 in [13, we have

$$
\left\|R_{3}\right\|_{L^{\infty}\left(\Omega \times \mathcal{S}^{1}\right)} \leq \frac{C(\Omega)}{\epsilon^{3}}\left\|\mathcal{L} R_{3}\right\|_{L^{\infty}\left(\Omega \times \mathcal{S}^{1}\right)} \leq \frac{C(\Omega)}{\epsilon^{3}}\left(C \epsilon^{4}\right) \leq C(\Omega) \epsilon .
$$

Hence, we have

$$
\left\|u^{\epsilon}-\sum_{k=0}^{3} \epsilon^{k} U_{k}^{\epsilon}-\sum_{k=0}^{3} \epsilon^{k} \mathscr{U}_{+, k}^{\epsilon}-\sum_{k=0}^{3} \epsilon^{k} \mathscr{U}_{-, k}^{\epsilon}\right\|_{L^{\infty}\left(\Omega \times \mathcal{S}^{1}\right)}=O(\epsilon) .
$$

Since it is easy to see

$$
\left\|\sum_{k=1}^{3} \epsilon^{k} U_{k}^{\epsilon}+\sum_{k=1}^{3} \epsilon^{k} \mathscr{U}_{+, k}^{\epsilon}+\sum_{k=1}^{3} \epsilon^{k} \mathscr{U}_{-, k}^{\epsilon}\right\|_{L^{\infty}\left(\Omega \times \mathcal{S}^{1}\right)}=O(\epsilon),
$$

our result naturally follows. This completes the proof of (1.12).

Step 5: Counterexample of the expansion.

Proof of (1.13). It is divided it into the following steps.

(1). The classical Milne problem.

By (2.28), the solution $f_{ \pm, 0}$ satisfies the Milne problem

$$
\left\{\begin{aligned}
\pm \sin (\theta+\xi) \frac{\partial f_{ \pm, 0}}{\partial \eta_{ \pm}}+f_{ \pm, 0}-\bar{f}_{ \pm, 0} & =0 \\
f_{ \pm, 0}(0, \theta, \xi) & =g_{ \pm}(\theta, \xi) \text { for } \pm \sin (\theta+\xi)>0 \\
\lim _{\eta_{ \pm} \rightarrow \infty} f_{ \pm, 0}\left(\eta_{ \pm}, \theta, \xi\right) & =f_{ \pm, 0}(\infty, \theta)
\end{aligned}\right.
$$

For convenience of comparison, we make the substitution $\phi=\theta+\xi$ to obtain

$$
\left\{\begin{aligned}
\pm \sin \phi \frac{\partial f_{ \pm, 0}}{\partial \eta_{ \pm}}+f_{ \pm, 0}-\bar{f}_{ \pm, 0} & =0 \\
f_{ \pm, 0}(0, \theta, \phi) & =g_{ \pm}(\theta, \phi) \text { for } \pm \sin \phi>0 \\
\lim _{\eta_{ \pm} \rightarrow \infty} f_{ \pm, 0}\left(\eta_{ \pm}, \theta, \phi\right) & =f_{ \pm, 0}(\infty, \theta) .
\end{aligned}\right.
$$

Assume (1.13) is incorrect, i.e.

$$
\lim _{\epsilon \rightarrow 0}\left\|\left(U_{0}+\mathscr{U}_{+, 0}+\mathscr{U}_{-, 0}\right)-\left(U_{0}^{\epsilon}+\mathscr{U}_{+, 0}^{\epsilon}+\mathscr{U}_{-, 0}^{\epsilon}\right)\right\|_{L^{\infty}}=0 .
$$

Since the boundary $g_{ \pm}(\phi)$ independent of $\theta$, by (2.28) and (2.53), it is obvious the limit of zeroth order boundary layer $f_{ \pm, 0}(\infty, \theta)$ and $f_{ \pm, 0}^{\epsilon}(\infty, \theta)$ satisfy $f_{ \pm, 0}(\infty, \theta)=C_{ \pm, 1}$ and $f_{ \pm, 0}^{\epsilon}(\infty, \theta)=C_{ \pm, 2}$ for some constant $C_{ \pm, 1}$ and $C_{ \pm, 2}$ independent of $\theta$. It is easy to see $C_{+, 1}=C_{+, 2}=0$. By (2.29) and (2.54), we can derive the interior solutions are smooth in the domain $\Omega$. Hence, for $\left|\eta_{-}\right| \leq \epsilon$ we may further derive

$$
\lim _{\epsilon \rightarrow 0}\left\|\left(f_{-, 0}(\infty)+\mathscr{U}_{-, 0}\right)-\left(f_{-, 0}^{\epsilon}(\infty)+\mathscr{U}_{-, 0}^{\epsilon}\right)\right\|_{L^{\infty}}=0 .
$$


For $0 \leq \eta \leq 1 /(2 \epsilon)\left(R_{+}-R_{-}\right)$, we have $\psi_{0}=1$, which means $f_{-, 0}=\mathscr{U}_{-, 0}+f_{-, 0}(\infty)$ and $f_{-, 0}^{\epsilon}=\mathscr{U}_{-, 0}^{\epsilon}+f_{-, 0}^{\epsilon}(\infty)$ on $[0, \epsilon]$. Define $u=f_{-, 0}+2, U=f_{-, 0}^{\epsilon}+2$ and $G_{-}=g_{-}+2=\cos \phi+2$, using the substitution and notation in Section 3, then $u(\eta, \phi)$ satisfies the equation

$$
\left\{\begin{aligned}
\sin \phi \frac{\partial u}{\partial \eta}+u-\bar{u} & =0 \\
u(0, \phi) & =G(\phi) \text { for } \sin \phi>0 \\
\lim _{\eta \rightarrow \infty} u(\eta, \phi) & =2+f_{0}(\infty)
\end{aligned}\right.
$$

and $U(\eta, \phi)$ satisfies the equation

$$
\left\{\begin{aligned}
\sin \phi \frac{\partial U}{\partial \eta}-F(\epsilon ; \eta) \cos \phi \frac{\partial U}{\partial \phi}+U-\bar{U} & =0 \\
U(0, \phi) & =G(\phi) \text { for } \sin \phi>0 \\
\lim _{\eta \rightarrow \infty} U(\eta, \phi) & =2+f_{0}^{\epsilon}(\infty)
\end{aligned}\right.
$$

Based on (5.29), we have

$$
\lim _{\epsilon \rightarrow 0}\|U(\eta, \phi)-u(\eta, \phi)\|_{L^{\infty}}=0 .
$$

Then it naturally implies

$$
\lim _{\epsilon \rightarrow 0}\|\bar{U}(\eta)-\bar{u}(\eta)\|_{L^{\infty}}=0 .
$$

(2) Continuity of $\bar{u}$ and $\bar{U}$ at $\eta=0$.

For the problem (5.30), we have for any $r_{0}>0$

$$
|\bar{u}(\eta)-\bar{u}(0)| \leq \frac{1}{2 \pi}\left(\int_{\sin \phi \leq r_{0}}|u(\eta, \phi)-u(0, \phi)| \mathrm{d} \phi+\int_{\sin \phi \geq r_{0}}|u(\eta, \phi)-u(0, \phi)| \mathrm{d} \phi\right) .
$$

Since we have shown $u \in L^{\infty}([0, \infty) \times[-\pi, \pi))$, then for any $\delta>0$, we can take $r_{0}$ sufficiently small such that

$$
\frac{1}{2 \pi} \int_{\sin \phi \leq r_{0}}|u(\eta, \phi)-u(0, \phi)| \mathrm{d} \phi \leq \frac{C}{2 \pi} \arcsin r_{0} \leq \frac{\delta}{2} .
$$

For fixed $r_{0}$ satisfying above requirement, we estimate the integral on $\sin \phi \geq r_{0}$. By Ukai's trace theorem, $u(0, \phi)$ is well-defined in the domain $\sin \phi \geq r_{0}$ and is continuous. Also, by consider the relation

$$
\frac{\partial u}{\partial \eta}(0, \phi)=\frac{\bar{u}(0)-u(0, \phi)}{\sin \phi}
$$

we can obtain in this domain $\partial_{\eta} u$ is bounded, which further implies $u(\eta, \phi)$ is uniformly continuous at $\eta=0$. Then there exists $\delta_{0}>0$ sufficiently small, such that for any $0 \leq \eta \leq \delta_{0}$, we have

$$
\frac{1}{2 \pi} \int_{\sin \phi \geq r_{0}}|u(\eta, \phi)-u(0, \phi)| \mathrm{d} \phi \leq \frac{1}{2 \pi} \int_{\sin \phi \geq r_{0}} \frac{\delta}{2} \mathrm{~d} \phi \leq \frac{\delta}{2} .
$$

In summary, we have shown for any $\delta>0$, there exists $\delta_{0}>0$ such that for any $0 \leq \eta \leq \delta_{0}$,

$$
|\bar{u}(\eta)-\bar{u}(0)| \leq \frac{\delta}{2}+\frac{\delta}{2}=\delta .
$$

Hence, $\bar{u}(\eta)$ is continuous at $\eta=0$. By a similar argument along the characteristics, we can show $\bar{U}(\eta, \phi)$ is also continuous at $\eta=0$.

In the following, by the continuity, we assume for arbitrary $\delta>0$, there exists a $\delta_{0}>0$ such that for any $0 \leq \eta \leq \delta_{0}$, we have

$$
\begin{aligned}
|\bar{u}(\eta)-\bar{u}(0)| & \leq \delta \\
|\bar{U}(\eta)-\bar{U}(0)| & \leq \delta .
\end{aligned}
$$

(3). The $\epsilon$-Milne formulation.

We consider the solution at a specific point $(\eta, \phi)=(n \epsilon, \epsilon)$ for some fixed $0<n \leq 1 / 2$. The solution along the characteristics can be rewritten as follows:

$$
u(n \epsilon, \epsilon)=G(\epsilon) \mathrm{e}^{-\frac{1}{\sin \epsilon} n \epsilon}+\int_{0}^{n \epsilon} \mathrm{e}^{-\frac{1}{\sin \epsilon}(n \epsilon-\kappa)} \frac{1}{\sin \epsilon} \bar{u}(\kappa) \mathrm{d} \kappa,
$$




$$
U(n \epsilon, \epsilon)=G\left(\epsilon_{0}\right) \mathrm{e}^{-\int_{0}^{n \epsilon} \frac{1}{\sin \phi(\zeta)} \mathrm{d} \zeta}+\int_{0}^{n \epsilon} \mathrm{e}^{-\int_{\kappa}^{n \epsilon} \frac{1}{\sin \phi(\zeta)} \mathrm{d} \zeta} \frac{1}{\sin \phi(\kappa)} \bar{U}(\kappa) \mathrm{d} \kappa,
$$

where we have the conserved energy along the characteristics

$$
E(\eta, \phi)=\cos \phi \mathrm{e}^{V(\eta)},
$$

in which $\left(0, \epsilon_{0}\right)$ and $(\zeta, \phi(\zeta))$ are in the same characteristics of $(n \epsilon, \epsilon)$.

(4) Estimates of (5.41).

We turn to the Milne problem for $u$. We have the natural estimate

$$
\begin{aligned}
\int_{0}^{n \epsilon} \mathrm{e}^{-\frac{1}{\sin \epsilon}(n \epsilon-\kappa)} \frac{1}{\sin \epsilon} \mathrm{d} \kappa & =\int_{0}^{n \epsilon} \mathrm{e}^{-\frac{1}{\epsilon}(n \epsilon-\kappa)} \frac{1}{\epsilon} \mathrm{d} \kappa+o(\epsilon) \\
& =\mathrm{e}^{-n} \int_{0}^{n \epsilon} \mathrm{e}^{\frac{\kappa}{\epsilon}} \frac{1}{\epsilon} \mathrm{d} \kappa+o(\epsilon) \\
& =\mathrm{e}^{-n} \int_{0}^{n} e^{\zeta} \mathrm{d} \zeta+o(\epsilon) \\
& =\left(1-\mathrm{e}^{-n}\right)+o(\epsilon) .
\end{aligned}
$$

Then for $0<\epsilon \leq \delta_{0}$, we have $|\bar{u}(0)-\bar{u}(\kappa)| \leq \delta$, which implies

$$
\begin{aligned}
\int_{0}^{n \epsilon} \mathrm{e}^{-\frac{1}{\sin \epsilon}(n \epsilon-\kappa)} \frac{1}{\sin \epsilon} \bar{u}(\kappa) \mathrm{d} \kappa & =\int_{0}^{n \epsilon} \mathrm{e}^{-\frac{1}{\sin \epsilon}(n \epsilon-\kappa)} \frac{1}{\sin \epsilon} \bar{u}(0) \mathrm{d} \kappa+O(\delta) \\
& =\left(1-\mathrm{e}^{-n}\right) \bar{u}(0)+o(\epsilon)+O(\delta) .
\end{aligned}
$$

For the boundary data term, it is easy to see

$$
G(\epsilon) \mathrm{e}^{-\frac{1}{\sin \epsilon} n \epsilon}=\mathrm{e}^{-n} G(\epsilon)+o(\epsilon)
$$

In summary, we have

$$
u(n \epsilon, \epsilon)=\left(1-\mathrm{e}^{-n}\right) \bar{u}(0)+\mathrm{e}^{-n} G(\epsilon)+o(\epsilon)+O(\delta) .
$$

(5). Approximation of (5.42).

We consider the $\epsilon$-Milne problem for $U$. For $\epsilon<<1$ sufficiently small, $\psi(\epsilon)=1$. Then we may estimate

$$
\cos \phi(\zeta) \mathrm{e}^{V(\zeta)}=\cos \epsilon \mathrm{e}^{V(n \epsilon)},
$$

which implies

$$
\cos \phi(\zeta)=\frac{1-\epsilon \zeta}{1-n \epsilon^{2}} \cos \epsilon
$$

and hence

$$
\sin \phi(\zeta)=\sqrt{1-\cos ^{2} \phi(\zeta)}=\sqrt{-\frac{\epsilon(n \epsilon-\zeta)\left(2-\epsilon \zeta-n \epsilon^{2}\right)}{\left(1-n \epsilon^{2}\right)^{2}} \cos ^{2} \epsilon+\sin ^{2} \epsilon} .
$$

For $\zeta \in[0, \epsilon]$ and $n \epsilon$ sufficiently small, by Taylor's expansion, we have

$$
\begin{aligned}
2-\epsilon \zeta-n \epsilon^{2} & =2+o(\epsilon), \\
\sin ^{2} \epsilon & =\epsilon^{2}+o\left(\epsilon^{3}\right), \\
\cos ^{2} \epsilon & =1-\epsilon^{2}+o\left(\epsilon^{3}\right) .
\end{aligned}
$$

Hence, we have

$$
\sin \phi(\zeta)=\sqrt{\epsilon(\epsilon-2 n \epsilon+2 \zeta)}+o\left(\epsilon^{2}\right) .
$$

Since $\sqrt{\epsilon(\epsilon-2 n \epsilon+2 \zeta)}=O(\epsilon)$, we can further estimate

$$
\begin{aligned}
\frac{1}{\sin \phi(\zeta)} & =\frac{1}{\sqrt{\epsilon(\epsilon-2 n \epsilon+2 \zeta)}}+o(1) \\
-\int_{\kappa}^{n \epsilon} \frac{1}{\sin \phi(\zeta)} \mathrm{d} \zeta & =-\left.\sqrt{\frac{\epsilon-2 n \epsilon+2 \zeta}{\epsilon}}\right|_{\kappa} ^{n \epsilon}+o(\epsilon)=\sqrt{\frac{\epsilon-2 n \epsilon+2 \kappa}{\epsilon}}-1+o(\epsilon) .
\end{aligned}
$$


Then we can easily derive the integral estimate

$$
\begin{aligned}
\int_{0}^{n \epsilon} \mathrm{e}^{-\int_{\kappa}^{n \epsilon} \frac{1}{\sin \phi(\zeta)} \mathrm{d} \zeta} \frac{1}{\sin \phi(\kappa)} \mathrm{d} \kappa & =\mathrm{e}^{-1} \int_{0}^{n \epsilon} \mathrm{e}^{\sqrt{\frac{\epsilon-2 n \epsilon+2 \kappa}{\epsilon}}} \frac{1}{\sqrt{\epsilon(\epsilon-2 n \epsilon+2 \kappa)}} \mathrm{d} \kappa+o(\epsilon) \\
& =\frac{1}{2} \mathrm{e}^{-1} \int_{(1-2 n) \epsilon}^{\epsilon} \mathrm{e}^{\sqrt{\frac{\sigma}{\epsilon}}} \frac{1}{\sqrt{\epsilon \sigma}} \mathrm{d} \sigma+o(\epsilon) \\
& =\frac{1}{2} \mathrm{e}^{-1} \int_{1-2 n}^{1} \mathrm{e}^{\sqrt{\rho}} \frac{1}{\sqrt{\rho}} \mathrm{d} \rho+o(\epsilon) \\
& =\mathrm{e}^{-1} \int_{\sqrt{1-2 n}}^{1} \mathrm{e}^{t} \mathrm{~d} t+o(\epsilon) \\
& =\left(1-\mathrm{e}^{\sqrt{1-2 n}-1}\right)+o(\epsilon) .
\end{aligned}
$$

Then for $0<\epsilon \leq \delta_{0}$, we have $|\bar{U}(0)-\bar{U}(\kappa)| \leq \delta$, which implies

$$
\begin{aligned}
\int_{0}^{n \epsilon} \mathrm{e}^{-\int_{\kappa}^{n \epsilon} \frac{1}{\sin \phi(\zeta)} \mathrm{d} \zeta} \frac{1}{\sin \phi(\kappa)} \bar{U}(\kappa) \mathrm{d} \kappa & =\int_{0}^{n \epsilon} \mathrm{e}^{-\int_{\kappa}^{n \epsilon} \frac{1}{\sin \phi(\zeta)} \mathrm{d} \zeta} \frac{1}{\sin \phi(\kappa)} \bar{U}(0) \mathrm{d} \kappa+O(\delta) \\
& =\left(1-\mathrm{e}^{\sqrt{1-2 n}-1}\right) \bar{U}(0)+o(\epsilon)+O(\delta) .
\end{aligned}
$$

For the boundary data term, since $G(\phi)$ is $C^{1}$, a similar argument shows

$$
G\left(\epsilon_{0}\right) \mathrm{e}^{-\int_{0}^{n \epsilon} \frac{1}{\sin \phi(\zeta)} \mathrm{d} \zeta}=\mathrm{e}^{\sqrt{1-2 n}-1} G(\sqrt{1-2 n} \epsilon)+o(\epsilon) .
$$

Therefore, we have

$$
U(n \epsilon, \epsilon)=\left(1-\mathrm{e}^{\sqrt{1-2 n}-1}\right) \bar{U}(0)+\mathrm{e}^{\sqrt{1-2 n}-1} G(\sqrt{1-2 n} \epsilon)+o(\epsilon)+O(\delta) .
$$

(6). Approximation of (1.13).

In summary, we have the estimate

$$
\begin{aligned}
u(n \epsilon, \epsilon) & =\left(1-\mathrm{e}^{-n}\right) \bar{u}(0)+\mathrm{e}^{-n} G(\epsilon)+o(\epsilon)+O(\delta), \\
U(n \epsilon, \epsilon) & =U(n \epsilon, \epsilon)=\left(1-\mathrm{e}^{\sqrt{1-2 n}-1}\right) \bar{U}(0)+\mathrm{e}^{\sqrt{1-2 n}-1} G(\sqrt{1-2 n} \epsilon)+o(\epsilon)+O(\delta) .
\end{aligned}
$$

The boundary data is $G=\cos \phi+2$. Then by the maximum principle in Theorem 3.14, we can achieve $1 \leq u(0, \phi) \leq 3$ and $1 \leq U(0, \phi) \leq 3$. Since

$$
\begin{aligned}
\bar{u}(0) & =\frac{1}{2 \pi} \int_{-\pi}^{\pi} u(0, \phi) \mathrm{d} \phi=\frac{1}{2 \pi} \int_{\sin \phi>0} u(0, \phi) \mathrm{d} \phi+\frac{1}{2 \pi} \int_{\sin \phi<0} u(0, \phi) \mathrm{d} \phi \\
& =\frac{1}{2 \pi} \int_{\sin \phi>0}(2+\cos \phi) \mathrm{d} \phi+\frac{1}{2 \pi} \int_{\sin \phi<0} u(0, \phi) \mathrm{d} \phi \\
& =2+\frac{1}{2 \pi} \int_{\sin \phi>0} \cos \phi \mathrm{d} \phi+\frac{1}{2 \pi} \int_{\sin \phi<0} u(0, \phi) \mathrm{d} \phi,
\end{aligned}
$$

we naturally obtain $3 / 2 \leq \bar{u}(0) \leq 5 / 2$. Similarly, we can obtain $3 / 2 \leq \bar{U}(0) \leq 5 / 2$. Furthermore, for $\epsilon$ sufficiently small, we have

$$
\begin{aligned}
G(\sqrt{1-2 n} \epsilon) & =3+o(\epsilon), \\
G(\epsilon) & =3+o(\epsilon) .
\end{aligned}
$$

Hence, we can obtain

$$
\begin{aligned}
u(n \epsilon, \epsilon) & =\bar{u}(0)+\mathrm{e}^{-n}(-\bar{u}(0)+3)+o(\epsilon)+O(\delta), \\
U(n \epsilon, \epsilon) & =\bar{U}(0)+\mathrm{e}^{\sqrt{1-2 n}-1}(-\bar{U}(0)+3)+o(\epsilon)+O(\delta) .
\end{aligned}
$$

Then we can see $\lim _{\epsilon \rightarrow 0}\|\bar{U}(0)-\bar{u}(0)\|_{L^{\infty}}=0$ naturally leads to $\lim _{\epsilon \rightarrow 0}\|(-\bar{u}(0)+3)-(-\bar{U}(0)+3)\|_{L^{\infty}}=0$. Also, we have $-\bar{u}(0)+3=O(1)$ and $-\bar{U}(0)+3=O(1)$. Due to the smallness of $\epsilon$ and $\delta$, and also $\mathrm{e}^{-n} \neq \mathrm{e}^{\sqrt{1-2 n}-1}$, we can obtain

$$
|U(n \epsilon, \epsilon)-u(n \epsilon, \epsilon)|=O(1) .
$$

However, above result contradicts our assumption that $\lim _{\epsilon \rightarrow 0}\|U(\eta, \phi)-u(\eta, \phi)\|_{L^{\infty}}=0$ for any $(\eta, \phi)$. This completes the proof of (1.13). 


\section{REFERENCES}

[1] A. Bensoussan, J.-L. Lions, and G. C. Papanicolaou, Boundary layers and homogenization of transport processes, Publ. Res. Inst. Math. Sci., 15 (1979), pp. 53-157.

[2] C. Cercignani, R. Illner, and M. Pulvirenti, The mathematical theory of dilute gases, Springer-Verlag, New York, 1994.

[3] R. Esposito, Y. Guo, C. Kim, and R. Marra, Non-isothermal boundary in the Boltzmann theory and Fourier law, Comm. Math. Phys., 323 (2013), pp. 177-239.

[4] E. W. LARSEn, A functional-analytic approach to the steady, one-speed neutron transport equation with anisotropic scattering, Comm. Pure Appl. Math., 27 (1974), pp. 523-545.

[5] - Solutions of the steady, one-speed neutron transport equation for small mean free paths, J. Mathematical Phys., 15 (1974), pp. 299-305.

[6] - Neutron transport and diffusion in inhomogeneous media I., J. Mathematical Phys., 16 (1975), pp. $1421-1427$.

[7] — - Asymptotic theory of the linear transport equation for small mean free paths II., SIAM J. Appl. Math., 33 (1977), pp. 427-445.

[8] E. W. Larsen and J. D'Arruda, Asymptotic theory of the linear transport equation for small mean free paths I., Phys. Rev., 13 (1976), pp. 1933-1939.

[9] E. W. Larsen and G. J. HabetLer, A functional-analytic derivation of Case's full and half-range formulas, Comm. Pure Appl. Math., 26 (1973), pp. 525-537.

[10] E. W. Larsen and J. B. Keller, Asymptotic solution of neutron transport problems for small mean free paths, J. Mathematical Phys., 15 (1974), pp. 75-81.

[11] E. W. Larsen And P. F. Zweifel, On the spectrum of the linear transport operator, J. Mathematical Phys., 15 (1974), pp. 1987-1997.

[12] — Steady, one-dimensional multigroup neutron transport with anisotropic scattering, J. Mathematical Phys., 17 (1976), pp. 1812-1820.

[13] L. Wu AND Y. Guo, Geometric correction for diffusive expansion of steady neutron transport equation, Comm. Math. Phys., 336 (2015), pp. 1473-1553.

(L. $\mathrm{Wu})$

Department of Mathematical Sciences, Carnegie Mellon University

Pittsburgh, PA 15213, USA

E-mail address: 1wu2@andrew.cmu.edu

(X.F. Yang)

Department of Mathematics, MOE-LSC and Shl-MaC, Shanghai Jiao Tong University,

Shanghai, 200240, P.R. China

E-mail address: xf-yang@sjtu.edu.cn

(Y. Guo)

Division of Applied Mathematics, Brown University,

Providence, RI 02912, USA

E-mail address: Yan_Guo@brown.edu 